\title{
Probing the Versatility of Shape-Persistent Tetraphenylmethane Dendrimers by Modification of the Skeleton
}

\author{
Julio Ignacio Urzúa, Sandra Campana, Massimo Lazzari, Mercedes Torneiro
}

Submitted date: 07/10/2019 Posted date: 11/10/2019

Licence: CC BY-NC-ND 4.0

Citation information: Urzúa, Julio Ignacio; Campana, Sandra; Lazzari, Massimo; Torneiro, Mercedes (2019): Probing the Versatility of Shape-Persistent Tetraphenylmethane Dendrimers by Modification of the Skeleton. ChemRxiv. Preprint.

Tetraphenylmethane has emerged as a recurrent building block for advanced porous materials such as COFs, PAFs and hypercrosslinked polymers. Guided by a similar design principle, we have previously synthesized shape-persistent dendrimers with tetraphenylmethane nodes and ethynylene linkers. Here we report the generality of our approach by describing new dendritic architectures built from tetraphenylmethane. First, we prepared expanded dendrimers where the tetrahedral units are bonded through larger rigid rod spacers. Among the different synthetic strategies tested, the convergent route, with alternating steps of Pd-catalyzed Sonogashira coupling and alkyne activation by removal of TMS masking groups, efficiently afforded the first- and second-generation dendrimers. A second type of compounds having a linear diyne at the core is also described. The dendrimers of generations 1-2 were also synthesized by a convergent approach, with the diyne being assembled in the last step of the synthesis by a Glaser oxidative homocoupling of the corresponding dendrons bearing a terminal alkyne at the focal point. A third-generation dendrimer was also successfully prepared by a double-phase strategy.

File list (2)

JOC-Torneiro-2019-def.pdf (511.44 KiB)

view on ChemRxiv - download file

JOC-Torneiro-2019-def.docx (850.73 KiB)

view on ChemRxiv - download file 


\section{Probing the versatility of shape-persistent}

\section{tetraphenylmethane dendrimers by modification of the}

\section{skeleton.}

Julio I. Urzúa, a,† Sandra Campana, ${ }^{a}$ Massimo Lazzari ${ }^{\mathrm{b}}$ and Mercedes Torneiro ${ }^{\mathrm{a} *}$

aDepartamento de Química Orgánica, Facultade de Química, Universidade de Santiago de Compostela, 15782, Santiago de Compostela, Spain.

Centro Singular de Investigación en Química Biolóxica e Materiais Moleculares (CIQUS) and Departamento de Química Física, Universidade de Santiago de Compostela, 15782, Santiago de Compostela, Spain.

* E-mail: mercedes.torneiro@usc.es

† Present address: Comisión Chilena de Energía Nuclear, calle Nueva Bilbao 12501, Comuna de los Condes, 7600713, Santiago de Chile, Chile.

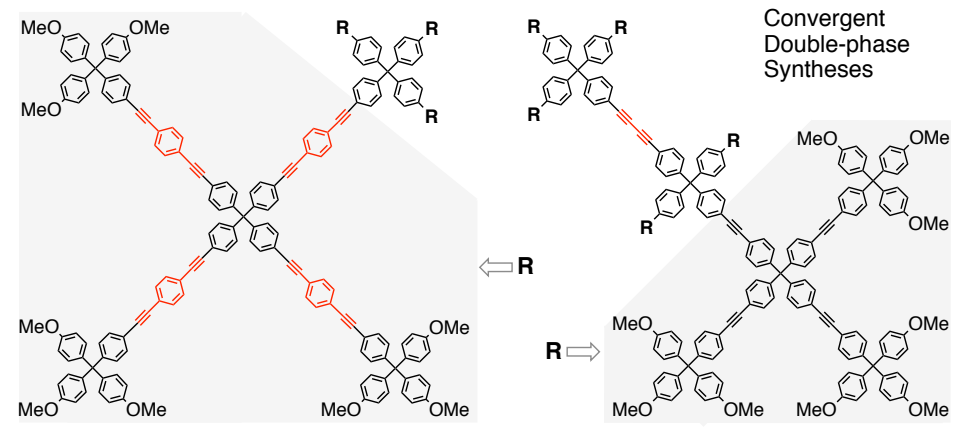


ABSTRACT. Tetraphenylmethane has emerged as a recurrent building block for advanced porous materials such as COFs, PAFs and hypercrosslinked polymers. Guided by a similar design principle, we have previously synthesized shape-persistent dendrimers with tetraphenylmethane nodes and ethynylene linkers. Here we report the generality of our approach by describing new dendritic architectures built from tetraphenylmethane. First, we prepared expanded dendrimers where the tetrahedral units are bonded through larger rigid rod spacers. Among the different synthetic strategies tested, the convergent route, with alternating steps of Pd-catalyzed Sonogashira coupling and alkyne activation by removal of TMS masking groups, efficiently afforded the first- and second-generation dendrimers. A second type of compounds having a linear diyne at the core is also described. The dendrimers of generations 1-2 were also synthesized by a convergent approach, with the diyne being assembled in the last step of the synthesis by a Glaser oxidative homocoupling of the corresponding dendrons bearing a terminal alkyne at the focal point. A third-generation dendrimer was also successfully prepared by a double-phase strategy.

\section{Introduction}

The tetrahedral tetravalent structure of $\mathrm{sp}^{3}$ carbon is responsible, in conjunction with the diversity of functional groups and the stability of the $\mathrm{C}-\mathrm{C}$ bond, of the structural and functional variety of organic compounds. Tetraphenylmethane can be considered as an expanded $\mathrm{sp}^{3}$ carbon that reproduces the same tetrahedral geometry in a larger structure when radially substituted, i.e. at the para positions of the aromatic rings. In fact, tetraphenylmethane is a rigid three-dimensional building block widely used to assemble the structural framework of artificial systems of considerable size. To name just a few, it has been employed to build tetrahedral structures, ${ }^{1}$ in self-assembled and supramolecular systems, ${ }^{2}$ as building block for light-emitting and antennae materials, ${ }^{3}$ in electronically active materials,${ }^{4}$ in liquid crystals,${ }^{5}$ for rotaxane stoppers ${ }^{6}$ and as core of shape-persistent dendrimers. ${ }^{7}$ A field of recent development where tetraphenylmethane has been extensively employed is that of organic porous materials. In particular, it has been used as building block for molecular cages, ${ }^{8}$ reticular materials (MOFs and COFs) ${ }^{9}$ and amorphous porous frameworks (microporous polymers, PAFs and related materials).${ }^{10}$ In this last case, 
the tetraphenylmethane units are hypercrosslinked either directly or through linear spacers to create diamond-related amorphous frameworks with internal voids or pores resulting from the radial stiffness and inefficient packing of the building blocks.

We have recently reported the synthesis and fluorescent properties of shape-persistent dendrimers with tetraphenylmethane nodes and ethynylene linkers of generations 1 and 2 (e.g. G1a, Fig. 1). ${ }^{11}$ The interest of these macromolecules lies in their privileged architectures, with a protein-like globular structure from the second generation. They have a hydrophobic interior with well-defined cavities and the polar terminal groups are effectively located at the periphery with reduced possibilities of backfolding. Being the soluble and structurally-regular analogs of microporous polymers, dendrimers of this type can be potentially used for separation technologies, for gas storage, in catalysis, sensing, and to build multichromophoric luminescent materials or nanomachines.

In this paper, we investigate the scope of the convergent, divergent and double-phase synthetic methodologies to access new members of this family of macromolecules. In addition, we show the versatility of this modular design by preparing dendrimers with modifications at their skeleton, either at the branches or the core. In the first case, our goal is the synthesis of molecules with an expanded structure, which can be accomplished by introducing larger rigid rod linkers between the tretraphenylmethane nodes. As an example, we selected for this work dendrimers G1b and G2b (Fig. 1), which have ethynylene-phenylene-ethynylene spacers. As a consequence, they have bigger size and molecular weight than the parent compounds (G1a and the analog second-generation dendrimer), while maintaining the shape-persistent structure. Three-dimensional models show that G1b has a star-like shape, whereas G2b has a globular structure with well-defined inner cavities of bigger volume then the parent compound. On the other side, we also describe in this work dendrimers G1c, G2c and G3c (Fig. 1), with a diyne core. The overall structure is more similar to the related tetraphenylmethane-cored prototypes (e.g. G1a), but with an ovoid instead of spherical globular shape resulting from the linear symmetry of the core. 

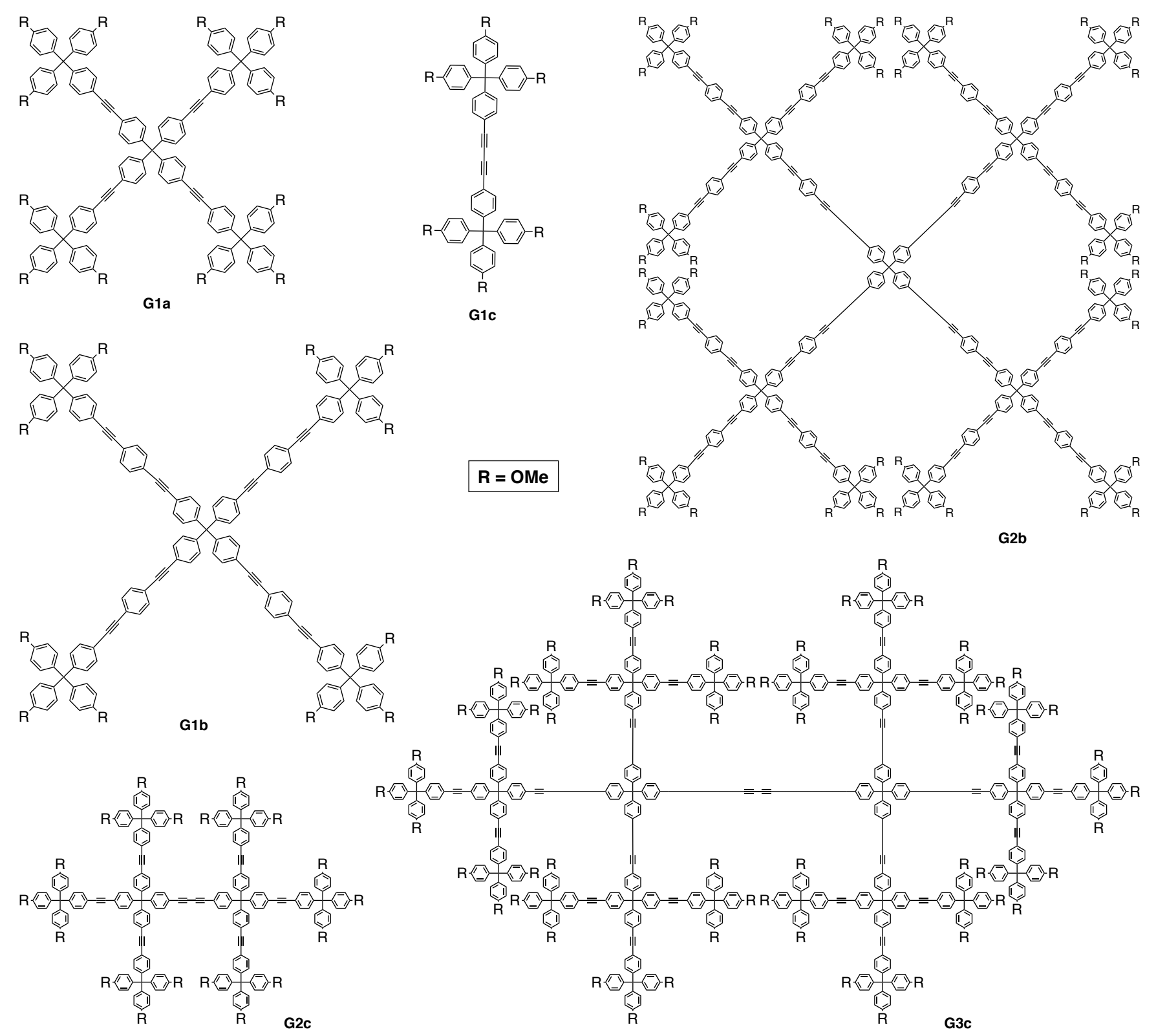

Figure 1. Structure of dendrimers of the $\mathbf{a}(\mathbf{G 1 a}), \mathbf{b}(\mathbf{G 1 b}, \mathbf{G 2 b})$ and $\mathbf{c}$ series $(\mathbf{G 1 c}, \mathbf{G 2 c}, \mathbf{G 3 c})$.

\section{Results and Discussion}

We started our work with the synthesis of the b series macromolecules. We envisaged that the expanded dendrimers could be easily accessed by a convergent route based on Sonogashira Pd-catalyzed couplings, previously demonstrated in our group for the prototype tetraphenylmethane dendrimers with ethynylene linkers. ${ }^{1 \mathrm{a} a}$ The first generation dendron or terminal unit $(\mathbf{4}$, Scheme 1$)$ was prepared by coupling of the alkyne $\mathbf{1}^{11}$ with the aryl bromide $2^{12}$ using $\mathrm{Pd}\left(\mathrm{PPh}_{3}\right)_{4}$ as catalyst in the presence of $\mathrm{PPh}_{3}, \mathrm{CuI}$ and $4 \AA$ molecular sieve in piperidine at $100{ }^{\circ} \mathrm{C}$, followed by removal of the TMS group at the focal point by 
treatment with sodium hydroxide in methanol. A new Sonogashira coupling of the dendron $\mathbf{4}$ with the core tetraiodide $\mathbf{5},{ }^{11,13}$ using the standard preceding conditions, afforded the first-generation dendrimer G1b in excellent $83 \%$ yield for the three steps.

\section{Scheme 1. Convergent route to dendrimers G1b and G2b.}

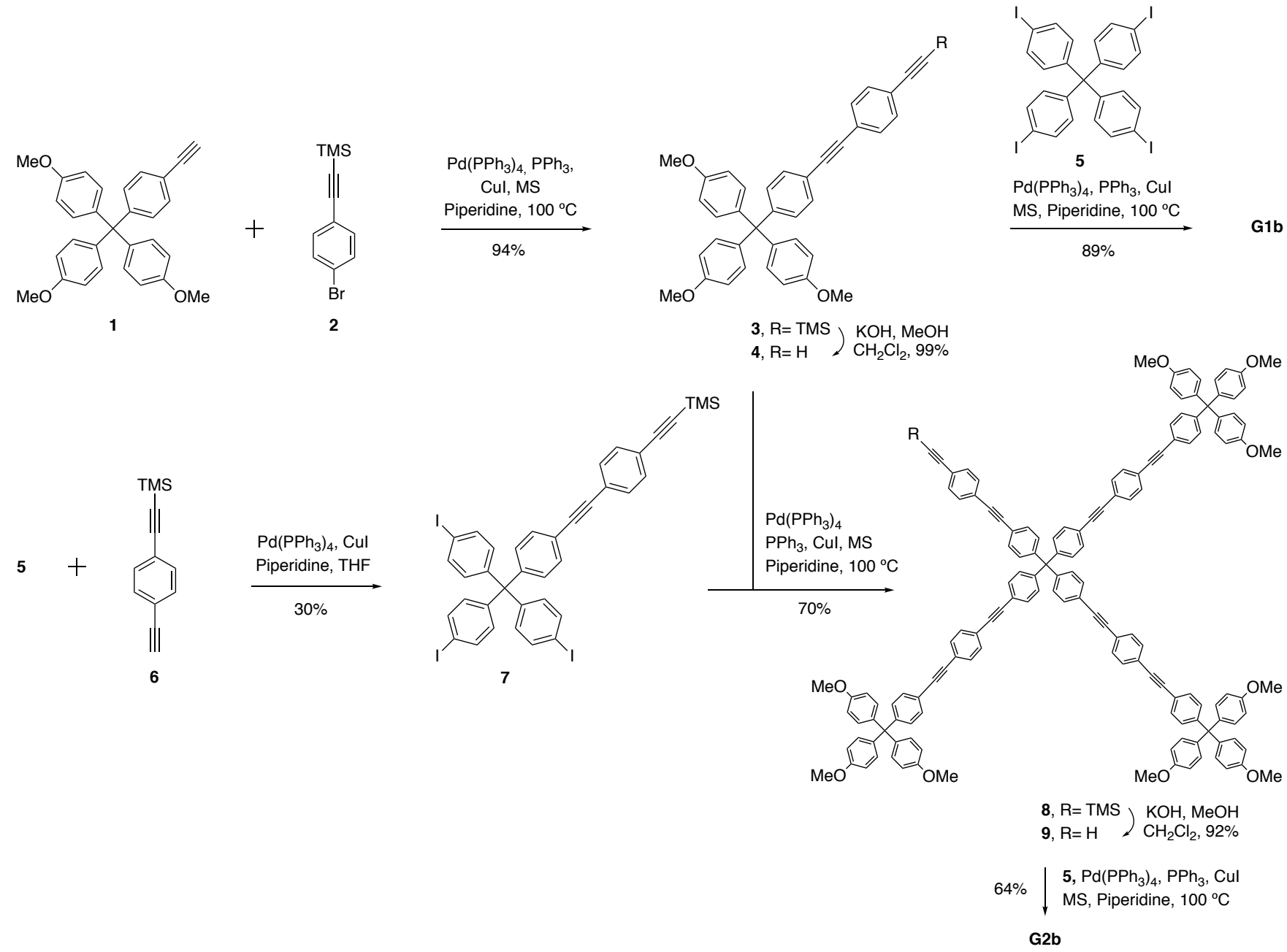

To address the synthesis of the second-generation dendrimer, we first prepared the branching unit 7 by coupling of the acetylene $6^{14}$ and the tetraiodide 5 using $\mathrm{Pd}\left(\mathrm{PPh}_{3}\right)_{4}, \mathrm{PPh}_{3}, \mathrm{CuI}$ and piperidine in $\mathrm{THF}$ at rt. The low yield in this reaction $(30 \%)$ is caused by the statistical reaction of the spacer with the aryl iodides of the tetravalent core. To avoid the formation of undesirable amounts of by-products resulting from coupling of multiple acetylenes to the core, an excess of the core building block $\mathbf{5}$ was used, which can be easily separated as the insoluble material in the reaction crude. The Pd-catalyzed coupling of the branching and the terminal units, 7 and 4, respectively, under standard conditions gave, in good yield, the protected second generation dendron, 8. Desilylation with $\mathrm{NaOH}$ and Sonogashira coupling with the core 
unit 5 afforded in good yield the second-generation dendrimer G2b. G1b and G2b were fully characterized by MALDI-TOF mass spectrometry and ${ }^{1} \mathrm{H} /{ }^{13} \mathrm{C}$ NMR spectroscopy and they showed a clean SEC trace with a single peak with retention times according to their molecular weights. A common property of this series of expanded dendrimers is their low solubility, that is useful for their isolation, as they precipitate in the reaction media as white solids. They are very stable, remaining unchanged after several days exposed to air on the bench. In addition, they show a characteristic luminescence that can be observed as a purple glow by illuminating a dilute solution under an UV lamp or as a bright blue spot upon UV irradiation of a sample on a TLC plate. They show a good stability,

Scheme 2. Divergent and double-phase strategies for the b series of dendrimers.

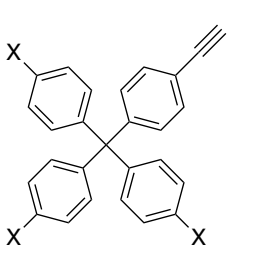

10

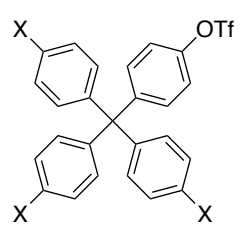

11
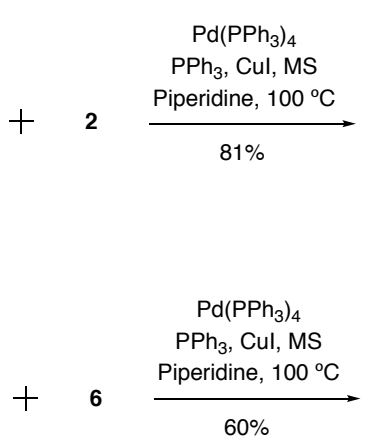

$60 \%$

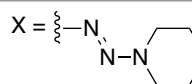

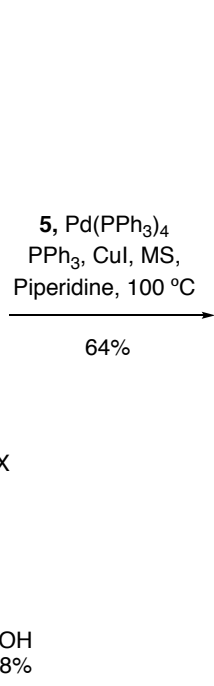
$\left.\begin{array}{l}\text { 12, } \mathrm{R}=\mathrm{TMS} \\ \text { 13, } \mathrm{R}=\mathrm{H}\end{array}\right) \begin{aligned} & \mathrm{KOH}, \mathrm{MeOH} \\ & \mathrm{CH}_{2} \mathrm{Cl}_{2}, 98 \%\end{aligned}$

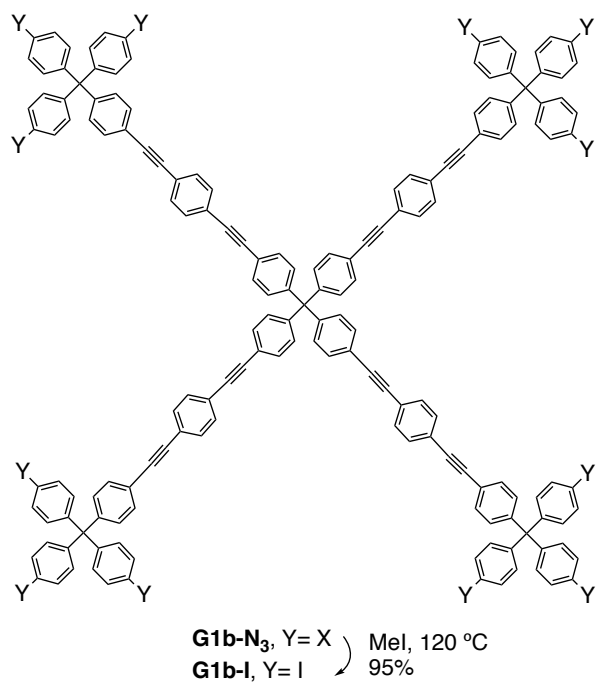

\begin{tabular}{r|l}
$\mathbf{4} / \mathbf{9}$ \\
$\begin{aligned} \mathrm{Pd}\left(\mathrm{PPh}_{3}\right)_{4} \\
\mathrm{Cul}\end{aligned}$ & $\begin{array}{l}\mathrm{PPh}_{3}, \mathrm{MS}, \text { Piperidine, } 100^{\circ} \mathrm{C} \\
\text { or } \\
\text { G2b } / \text { G3b }\end{array}$
\end{tabular}

At this point, we decided to apply our recently developed triazene-based divergent route ${ }^{11 \mathrm{~b}}$ to this series of dendrimers for comparative purposes. Therefore, we explored the divergent approach to G2b using as starting tetraphenylmethane building blocks the tris(triazene) derivatives $\mathbf{1 0}^{1 \mathrm{lb}}$ and $\mathbf{1 1}^{1 \mathrm{~b}}$ that were coupled to either spacer $\mathbf{2}$ or $\mathbf{6}$, respectively (Scheme 2). In both cases the desired protected expanded dendron 12 was obtained with good to moderate yields. Subsequent desilylation and coupling to tetraiodide $\mathbf{5}$ afforded the triazene-terminated first generation dendrimer $\mathbf{G 1 b}-\mathbf{N}_{\mathbf{3}}$ in $63 \%$ yield for the two steps, which was transformed in the iodo-terminated dendrimer G1b-I by treatment with $\mathrm{MeI}$ at $120{ }^{\circ} \mathrm{C}(95 \%$ yield). 
Unfortunately, our efforts to grow this dendrimer by coupling with dendron 4 were unsuccessful, yielding complex mixtures, in which compound G2b could not be detected. The reaction of G1b-I with dendron $\mathbf{9}$ was also tested in an attempt to prepare the corresponding third-generation dendrimer (G3b, structure not shown) by a double-stage method, but the desired dendrimer could not be detected in the resulting complex reaction mixtures.

Next, we turned our attention to the synthesis and characterization of the diyne-cored dendrimers of the c series. In addition to their interesting structure, these compounds should be easily accessible by Glaser oxidative homocoupling ${ }^{15}$ of the corresponding dendrons bearing a terminal alkyne at the focal point. Of the many conditions reported in the literature for this reaction, we selected those that were favorable to the dissolution of the substrates. We started by testing the reaction of $\mathbf{1}$ with different copper salts as catalyst in $\mathrm{CH}_{2} \mathrm{Cl}_{2},{ }^{16}$ but neither $\mathrm{CuI}$ or $\mathrm{CuCl}$ in combination with diethylamine, piperidine or pyrrolidine as bases and in the presence of oxygen gave the desired product G1c, and the starting material was recovered unaltered. Similar negative results were obtained when the reaction was carried out with Hua's conditions $^{17}-\mathrm{CuCl}$ and piperidine in toluene.

\section{Scheme 3. Convergent route to dendrimers G1c and G2c.}

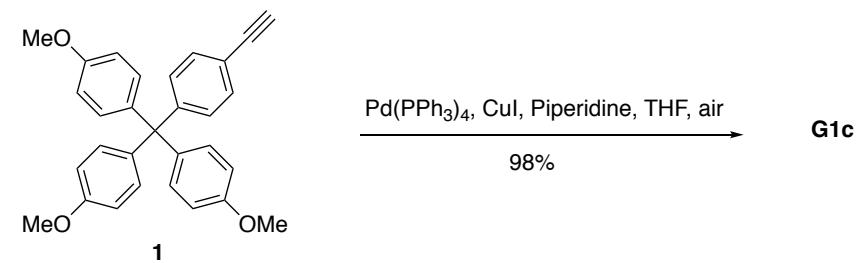

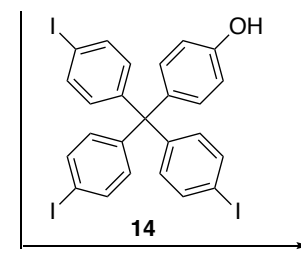

$\mathrm{Pd}\left(\mathrm{PPh}_{3}\right)_{4}, \mathrm{PPh}_{3}, \mathrm{Cul}, \mathrm{MS}$ Piperidine, $100{ }^{\circ} \mathrm{C}, 73 \%$

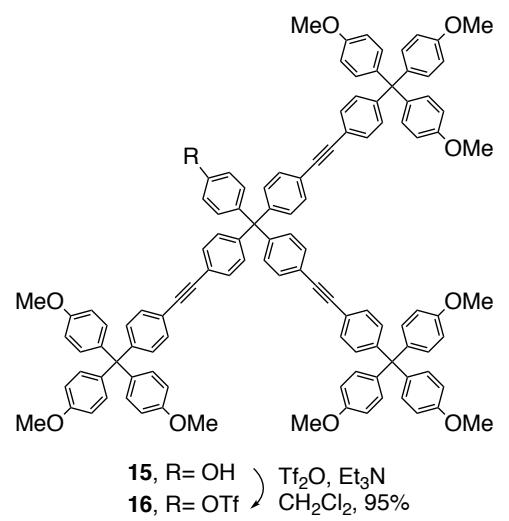

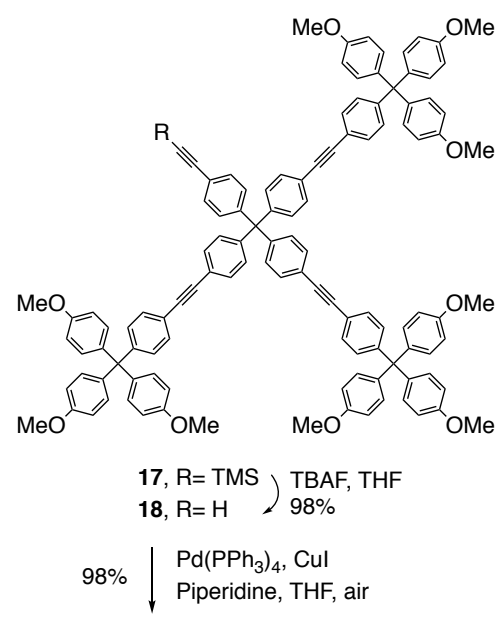

G2c 
Our observation during the synthesis of the tetraphenylmethane-cored dendrimers (e.g. G1a) that small amounts of the diyne-cored dendrimers were sometimes obtained as by-products, led us to essay the Sonogashira conditions under air at rt. So, the reaction of 1 with $\mathrm{Pd}\left(\mathrm{PPh}_{3}\right)_{4}(0.1$ eq. $), \mathrm{CuI}(0.1$ eq.) and piperidine (13 eq.) in THF in the presence of air afforded the desired dendrimer G1c in low yield, which was improved to $98 \%$ yield by increasing the amount of CuI to 2 eq. (Scheme 3). To undertake the synthesis of the dendrimer G2c, we first developed a new synthetic route to the protected secondgeneration dendron 17. Instead of coupling the spacer to the tetraiodide $\mathbf{5}$ with $\sim 30 \%$ yield, as in the previously reported method, ${ }^{11 a}$ and in the strategy followed to prepare $\mathbf{8}$ (Scheme 1), we reacted the terminal unit 1 to the known triiodide $\mathbf{1 4}^{6}$ to obtain the phenol 15 in $73 \%$ yield (Scheme 3). Treatment with triflic anhydride gave the intermediate triflate 16, which was coupled with the TMSA spacer to yield the desired protected dendron 17 ( $90 \%$ for the two steps). Desilylation to give the dendron $\mathbf{1 8}^{11 \mathrm{a}}$ followed by Glaser homocoupling in the above optimized conditions afforded the final second-generation dendrimer G2c in excellent $96 \%$ yield (two steps).

Once demonstrated the feasibility of the convergent method to access the c series of dendrimers, we considered worth to attempt the double-stage strategy for the synthesis of a dendrimer of third generation. The Glaser homocoupling of the tris(triazene) $\mathbf{1 0}$ gave the triazene terminated dendrimer $\mathbf{G 1 c}-\mathbf{N}_{\mathbf{3}}$ in excellent yield (Scheme 4). Treatment with methyl iodide afforded the hexaiodide G1c-I that worked as a supercore for dendrimer groth by Sonogashira reaction with dendron 18. In this case, the double-stage coupling proceeded smoothly to give the dendrimer G3c in $66 \%$ yield for the last two steps.

\section{Scheme 4. Double-phase synthesis of G3c.}




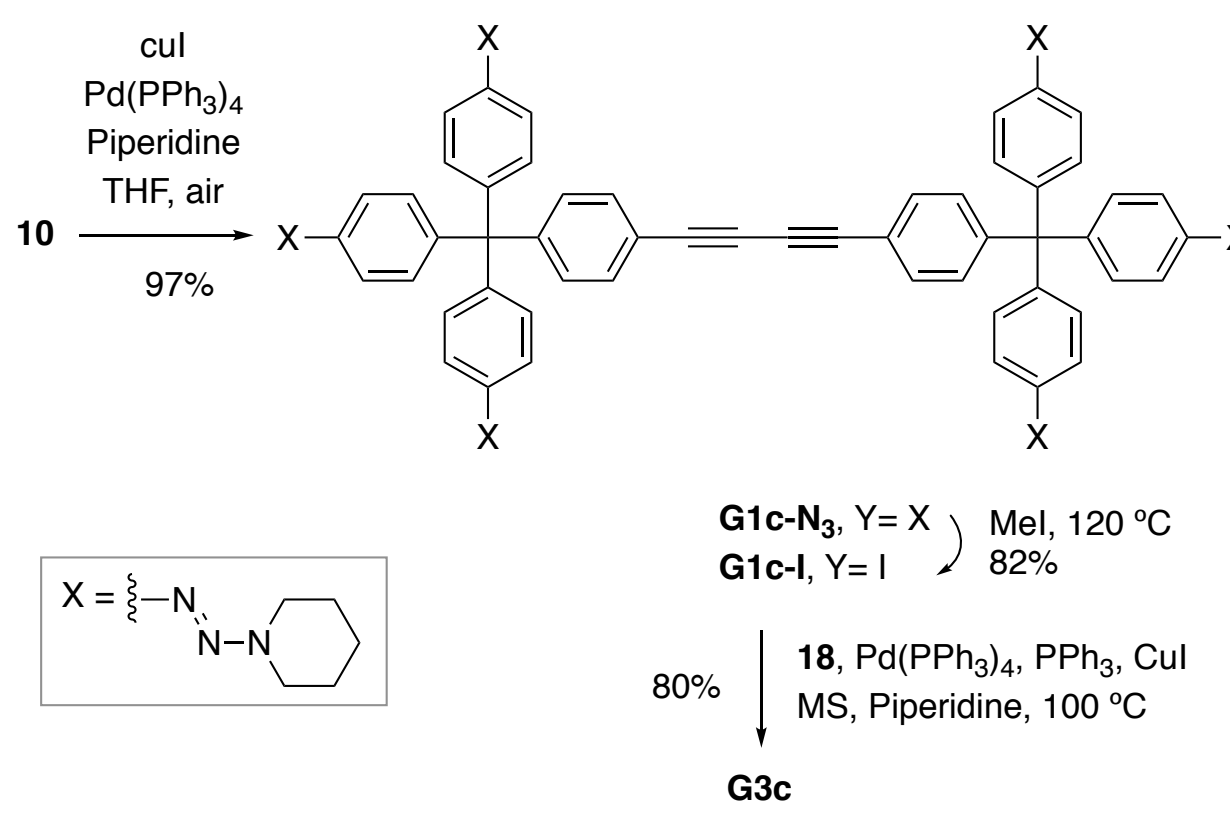

Dendrimers G1c, G2c and G3c were conveniently characterized ( ${ }^{1} \mathrm{H}$ and ${ }^{13} \mathrm{C}$ NMR, MALDI-TOF MS) and showed a clean SEC trace with retention times according to their sizes. They are stable white solids compounds with good solubility in common solvents (THF, $\mathrm{CH}_{2} \mathrm{Cl}_{2}, \mathrm{CHCl}_{3}$ ). By visual inspection they do not show luminiscent properties as in the case of the previous series.

In conclusion, we have shown that tetraphenylmethane-based shape persistent dendrimers have very versatile modular structures that are accessible by different synthetic methodologies. In this work we have prepared two new series of compounds with unique geometrical characteristics, either with a expanded structure or with a diyne core. Both families were easily prepared by a convergent strategy. Whereas the convergent and double-phase routes were unsuccessful for the synthesis of $\mathbf{G} \mathbf{2} \mathbf{b}$ and $\mathbf{G 3} \mathbf{b}$, respectively, G3b was efficiently prepared by a double-stage methodology. The chemistry involved in those syntheses is basically an iterative process based on Pd-catalyzed Sonogashira couplings of terminal alkynes with aryl iodides and triflates, with alternating steps of activation of masked groups. In the case of the $\mathbf{c}$ series there is also a key step consisting of a Glaser homodimerization to assemble the core diyne. This is the first example of a double-stage strategy for rigid tetraphenylmethane dendrimers, and also we report the first dendrimer of third generation of this class. Further studies are being carried out in our laboratories to evaluate the properties (e.g. porosity, fluorescence) of these new macromolecular materials, which will be reported in future papers. 


\section{Experimental Section ${ }^{18}$}

Protected dendron 3. A Schlenk tube equipped with a stirring bar was charged with $\mathbf{1}^{11}$ (200 $\mathrm{mg}, 0.46$ mmol), $2^{12}$ (175 mg, $\left.0.69 \mathrm{mmol}\right), \mathrm{CuI}(4 \mathrm{mg}, 0.023 \mathrm{mmol}), \mathrm{Ph}_{3} \mathrm{P}(6 \mathrm{mg}, 0.023 \mathrm{mmol})$ and $\left(\mathrm{Ph}_{3} \mathrm{P}\right)_{4} \mathrm{Pd}(27$ $\mathrm{mg}, 0.023 \mathrm{mmol})$. After vacuum-drying, activated $4 \AA$ molecular sieves and piperidine $(15 \mathrm{~mL})$ were added under argon. The Schlenk tube was sealed with a teflon screw cap and the reaction mixture was stirred at $100{ }^{\circ} \mathrm{C}$ during $12 \mathrm{~h}$ and then it was allowed to reach rt. The mixture was concentrated and the residue was dissolved in $\mathrm{CH}_{2} \mathrm{Cl}_{2}(20 \mathrm{~mL})$ and washed with a saturated solution of $\mathrm{NH}_{4} \mathrm{Cl}(3 \times 15 \mathrm{~mL})$. The organic layer was dried, filtered and concentrated and the residue was purified by flash chromatography $\left(\mathrm{SiO}_{2}, 5 \%\right.$ EtOAc/hexanes $)$ to give 3 as a white solid [267 $\mathrm{mg}, 0.44 \mathrm{mmol}, 94 \%, \mathrm{Rf}=$ 0.59 (20\% EtOAc/hexanes)]. ${ }^{1} \mathrm{H} \mathrm{NMR}\left(\mathrm{CDCl}_{3}, 250 \mathrm{MHz}\right): 7.43$ (4H, s, Ar), $7.41(2 \mathrm{H}, \mathrm{d}, \mathrm{J}=8.3 \mathrm{~Hz}, \mathrm{Ar})$ $7.20(2 \mathrm{H}, \mathrm{d}, J=8.3 \mathrm{~Hz}, \mathrm{Ar}), 7.10(6 \mathrm{H}, \mathrm{d}, J=8.8 \mathrm{~Hz}, \mathrm{Ar}), 6.80(6 \mathrm{H}, \mathrm{d}, J=8.8 \mathrm{~Hz} \mathrm{Ar}), 3.80(9 \mathrm{H}, \mathrm{s}, \mathrm{OMe})$, 0.27 (9H, s, TMS). ${ }^{13} \mathrm{C}$ NMR $\left(\mathrm{CDCl}_{3}, 63 \mathrm{MHz}\right): 157.1(\mathrm{C}), 147.8(\mathrm{C}), 138.6(\mathrm{C}), 131.6(\mathrm{CH}), 131.5(\mathrm{CH})$, $130.9(\mathrm{CH}), 130.3(\mathrm{CH}), 123.0(\mathrm{C}), 122.4(\mathrm{C}), 119.9(\mathrm{C}), 112.4(\mathrm{CH}), 104.3(\mathrm{C}), 95.8(\mathrm{C}), 90.9(\mathrm{C}), 88.6$ (C), $62.5(\mathrm{C}), 54.8\left(\mathrm{CH}_{3}\right), 0.44(\mathrm{TMS}) . \mathrm{MS}(\mathrm{EI}, \mathrm{m} / \mathrm{z}, \%): 606.0\left(\mathrm{M}^{+}, 75\right), 557.9(16), 499.0\left(\mathrm{M}^{+}-\mathrm{C}_{7} \mathrm{H}_{7} \mathrm{O}\right.$ 77), $333.0\left(\mathrm{M}^{+}-\mathrm{C}_{19} \mathrm{H}_{17} \mathrm{Si}, 100\right)$. HRMS (EI) calcd. for $\mathrm{C}_{41} \mathrm{H}_{38} \mathrm{O}_{3} \mathrm{Si}\left(\mathrm{M}^{+}\right)$606.2590, found 606.2590.

Dendron 4. To a solution of $3(100 \mathrm{mg}, 0.17 \mathrm{mmol})$ in $\mathrm{CH}_{2} \mathrm{Cl}_{2}(10 \mathrm{~mL})$ and $\mathrm{MeOH}(4 \mathrm{~mL})$ was added $\mathrm{KOH}(93 \mathrm{mg}, 1.7 \mathrm{mmol}$ ) under argon and the mixture was stirred at $\mathrm{rt}$ during $1 \mathrm{~h}$. The mixture was concentrated and the residue was dissolved in EtOAc $(20 \mathrm{~mL})$. The resulting solution was washed with brine $(3 \times 30 \mathrm{~mL})$, dried and concentrated to give a residue that was dissolved in $\mathrm{CH}_{2} \mathrm{Cl}_{2}$. Precipitation with hexanes gave 4 as a white solid [87 mg, $0.16 \mathrm{mmol}, 99 \%, \mathrm{Rf}=0.48$ (20\% EtOAc/hexanes)]. ${ }^{1} \mathrm{H}$ NMR $\left(\mathrm{CDCl}_{3}, 250 \mathrm{MHz}\right): 7.47(4 \mathrm{H}, \mathrm{s}, \mathrm{Ar}), 7.42(2 \mathrm{H}, \mathrm{d}, J=8.2 \mathrm{~Hz}, \mathrm{Ar}), 7.21(2 \mathrm{H}, \mathrm{d}, J=8.2 \mathrm{~Hz}, \mathrm{Ar}), 7.10(6 \mathrm{H}, \mathrm{d}$, $\left.J=8.7 \mathrm{~Hz}, \mathrm{Ar}_{\mathrm{OMe}}\right), 6.80\left(6 \mathrm{H}, \mathrm{d}, J=8.7 \mathrm{~Hz}, \mathrm{Ar}_{\mathrm{OMe}}\right), 3.79(9 \mathrm{H}, \mathrm{s}, \mathrm{OMe}), 3.18(1 \mathrm{H}, \mathrm{s}, \mathrm{C} \equiv \mathrm{C}-\mathrm{H}) .{ }^{13} \mathrm{C} \mathrm{NMR}$ $\left(\mathrm{CDCl}_{3}, 63 \mathrm{MHz}\right): 157.1(\mathrm{C}), 147.9(\mathrm{C}), 138.6(\mathrm{C}), 131.6(\mathrm{CH}), 131.5(\mathrm{CH}), 131.0(\mathrm{CH}), 130.6(\mathrm{CH})$, $130.3(\mathrm{CH}), 123.4(\mathrm{C}), 121.3(\mathrm{C}), 119.8(\mathrm{C}), 112.3(\mathrm{CH}), 91.0(\mathrm{C}), 88.4(\mathrm{C}), 82.9(\mathrm{C}), 78.5(\mathrm{C}), 62.5(\mathrm{C})$ $54.8\left(\mathrm{CH}_{3}\right) . \mathrm{MS}(\mathrm{EI}, \mathrm{m} / \mathrm{z}, \%): 534.0\left(\mathrm{M}^{+}, 78\right), 427.0\left(\mathrm{M}^{+}-\mathrm{C}_{7} \mathrm{H}_{7} \mathrm{O}, 85\right), 333.0\left(\mathrm{M}^{+}-\mathrm{C}_{16} \mathrm{H}_{9}, 100\right) . \mathrm{HRMS}(\mathrm{EI})$ calcd. for $\mathrm{C}_{38} \mathrm{H}_{30} \mathrm{O}_{3}\left(\mathrm{M}^{+}\right)$534.2195, found 534.2195. 
Dendrimer G1b. A Schlenk tube equipped with a stirring bar was charged with freshly prepared 4 (176 $\mathrm{mg}, 0.33 \mathrm{mmol}), \mathbf{5}^{11,13}(60 \mathrm{mg}, 0.073 \mathrm{mmol}), \mathrm{CuI}(6 \mathrm{mg}, 0.029 \mathrm{mmol}), \mathrm{Ph}_{3} \mathrm{P}(8 \mathrm{mg}, 0.029 \mathrm{mmol})$ and $\left(\mathrm{Ph}_{3} \mathrm{P}\right)_{4} \mathrm{Pd}(34 \mathrm{mg}, 0.029 \mathrm{mmol})$. After vacuum-drying, activated $4 \AA$ molecular sieves and piperidine $(15$ $\mathrm{mL})$ were added under argon. The Schlenk tube was sealed with a teflon screw cap and the reaction mixture was stirred at $100{ }^{\circ} \mathrm{C}$ during $12 \mathrm{~h}$. Then it was allowed to reach rt to give a white precipitate which was filtered and dissolved in hot $\mathrm{CH}_{2} \mathrm{Cl}_{2}(30 \mathrm{~mL})$. The resulting solution was allowed to slowly reach $\mathrm{rt}$ and a white precipitate was formed, which was filtered to give dendrimer G1b as a white solid [160 mg, $0.065 \mathrm{mmol}, 89 \%$ ]. ${ }^{1} \mathrm{H} \mathrm{NMR}\left(\mathrm{CDCl}_{3}, 250 \mathrm{MHz}\right): 7.46(32 \mathrm{H}, \mathrm{m}, \mathrm{Ar}), 7.19$ (16H, m, Ar), 7.08 $\left(24 \mathrm{H}, \mathrm{d}, J=8.7 \mathrm{~Hz}, \mathrm{Ar}_{\text {Оме }}\right), 6.78\left(24 \mathrm{H}, \mathrm{d}, J=8.8 \mathrm{~Hz} \mathrm{Ar}_{\mathrm{OMe}}\right), 3.79(36 \mathrm{H}, \mathrm{s}, \mathrm{OMe}) .{ }^{13} \mathrm{C} \mathrm{NMR}\left(\mathrm{CDCl}_{3}, 63\right.$ MHz): $157.4(\mathrm{C}), 148.1(\mathrm{C}), 145.8(\mathrm{C}), 138.8(\mathrm{C}), 131.8(\mathrm{CH}), 131.4(\mathrm{CH}), 130.8(\mathrm{CH}), 130.5(\mathrm{CH}), 123.1$ (C), $122.7(\mathrm{C}), 121.0(\mathrm{C}), 120.1(\mathrm{C}), 112.6(\mathrm{CH}), 91.1(\mathrm{C}), 90.6(\mathrm{C}), 89.5(\mathrm{C}), 88.9(\mathrm{C}), 64.4(\mathrm{C}), 62.7$ (C), $55.0\left(\mathrm{CH}_{3}\right)$. MS (MALDI-TOF, m/z): $2559\left(\mathrm{MAg}^{+}\right), 2450\left(\mathrm{M}^{+}\right), 2343\left(\mathrm{M}^{+}-\mathrm{C}_{7} \mathrm{H}_{7} \mathrm{O}\right), 1840\left(\mathrm{M}^{+}-\right.$ $\left.\mathrm{C}_{44} \mathrm{H}_{33} \mathrm{O}_{3}\right)$.

Trimehyl((4-((4-(tris(4-iodophenyl)methyl)phenyl)ethinyl)phenyl)ethinyl)silane (7). A mixture of $\mathbf{6}^{14}(77 \mathrm{mg}, 0.39 \mathrm{mmol}), \mathbf{5}^{11,13}(400 \mathrm{mg}, 0.49 \mathrm{mmol}), \mathrm{CuI}(9 \mathrm{mg}, 0.049 \mathrm{mmol})$ and $\left(\mathrm{Ph}_{3} \mathrm{P}\right)_{4} \mathrm{Pd}(56 \mathrm{mg}, 0.049$ mmol) was dissolved in THF $(50 \mathrm{~mL})$ by refluxing under argon. Then, the solution was allowed to reach rt and piperidine $(0.5 \mathrm{~mL}, 4.85 \mathrm{mmol})$ was added. After stirring for $12 \mathrm{~h}$, the mixture was concentrated, the residue was dissolved in $\mathrm{CH}_{2} \mathrm{Cl}_{2}(20 \mathrm{~mL})$ and the resulting solution was washed with a saturated solution of $\mathrm{NH}_{4} \mathrm{Cl}(3 \times 10 \mathrm{~mL})$. The organic phase was dried and concentrated and the residue was purified by flash chromatography $\left(\mathrm{SiO}_{2}\right.$, hexanes) to give 7 as a white solid [139 $\mathrm{mg}, 0.155 \mathrm{mmol}, 30 \%, \mathrm{Rf}=0.33$ (5\% $\mathrm{CH}_{2} \mathrm{Cl}_{2} /$ hexanes)]. ${ }^{1} \mathrm{H} \mathrm{NMR}\left(\mathrm{CDCl}_{3}, 250 \mathrm{MHz}\right): 7.59\left(6 \mathrm{H}, \mathrm{d}, J=8.0 \mathrm{~Hz}, \mathrm{Ar}_{\mathrm{I}}\right), 7.42(6 \mathrm{H}, \mathrm{m}, \mathrm{Ar}), 7.14$ $(2 \mathrm{H}, \mathrm{d}, J=8.1 \mathrm{~Hz}, \mathrm{Ar}), 6.91(6 \mathrm{H}, \mathrm{d}, J=8.0 \mathrm{~Hz} \mathrm{Ar}), 0.27(9 \mathrm{H}, \mathrm{s}, \mathrm{TMS}) .{ }^{13} \mathrm{C} \mathrm{NMR}\left(\mathrm{CDCl}_{3}, 63 \mathrm{MHz}\right): 145.7$ (C), $145.3(\mathrm{C}), 137.2(\mathrm{CH}), 132.8(\mathrm{CH}), 132.0(\mathrm{CH}), 131.5(\mathrm{CH}), 131.3(\mathrm{CH}), 130.8(\mathrm{CH}), 123.2(\mathrm{C})$, 123.1 (C), 121.4 (C), 104.7 (C), 96.5 (C), 92.6 (C), 90.8 (C), 89.8 (C), 64.4 (C), 0.08 (TMS). MS (EI, m/z, \%): 893.2 (M+ 100$), 690.4\left(\mathrm{M}^{+}-\mathrm{C}_{6} \mathrm{H}_{5} \mathrm{I}, 67\right)$. HRMS (EI) calcd. For $\mathrm{C}_{41} \mathrm{H}_{38} \mathrm{O}_{3} \mathrm{Si}^{+}\left(\mathrm{M}^{+}\right)$893.9173, found 893.9178. 
Protected dendron 8. A Schlenk tube equipped with a stirring bar was charged with 4 (330 mg, 0.62 mmol), 7 (158 mg, $0.18 \mathrm{mmol})$, CuI (5 mg, $0.026 \mathrm{mmol}), \mathrm{Ph}_{3} \mathrm{P}(7 \mathrm{mg}, 0.026 \mathrm{mmol})$ and $\left(\mathrm{Ph}_{3} \mathrm{P}\right)_{4} \mathrm{Pd}(61$ $\mathrm{mg}, 0.053 \mathrm{mmol})$. After vacuum-drying, activated $4 \AA$ molecular sieves and piperidine $(20 \mathrm{~mL})$ were added under argon. The Schlenk tube was sealed with a teflon screw cap and the reaction mixture was stirred at $100{ }^{\circ} \mathrm{C}$ during $12 \mathrm{~h}$ and then it was allowed to reach $\mathrm{rt}$. The mixture was concentrated and the residue was dissolved in $\mathrm{CH}_{2} \mathrm{Cl}_{2}(20 \mathrm{~mL})$ and washed with a saturated solution of $\mathrm{NH}_{4} \mathrm{Cl}(3 \mathrm{x} 15 \mathrm{~mL})$. The organic layer was dried, filtered and concentrated and the residue was purified by flash chromatography $\left(\mathrm{SiO}_{2}, 40 \% \mathrm{CH}_{2} \mathrm{Cl}_{2} /\right.$ hexanes) to give $\mathbf{8}$ as a white solid [260 $\mathrm{mg}, 0.12 \mathrm{mmol}, 70 \%, \mathrm{Rf}=$ $0.65\left(70 \%\right.$ toluene/ $\left.\left.\mathrm{CH}_{2} \mathrm{Cl}_{2}\right)\right] .{ }^{1} \mathrm{H} \mathrm{NMR}\left(\mathrm{CDCl}_{3}, 250 \mathrm{MHz}\right): 7.52-7.35(30 \mathrm{H}, \mathrm{m}, \mathrm{Ar}), 7.21(14 \mathrm{H}, \mathrm{m}, \mathrm{Ar})$, $7.10\left(18 \mathrm{H}, \mathrm{d}, J=8.8 \mathrm{~Hz}, \mathrm{Ar}_{\text {OMe }}\right), 6.80\left(18 \mathrm{H}, \mathrm{d}, J=8.8 \mathrm{~Hz} \mathrm{Ar}_{\mathrm{OMe}}\right), 3.80$ (27H, s, OMe), 0.27 (9H, s, TMS). ${ }^{13} \mathrm{C} \mathrm{NMR}\left(\mathrm{CDCl}_{3}, 63 \mathrm{MHz}\right): 157.4(\mathrm{C}), 148.1(\mathrm{C}), 145.9(\mathrm{C}), 138.9(\mathrm{C}), 131.8(\mathrm{CH}), 131.4(\mathrm{CH}), 131.0$ $(\mathrm{CH}), 130.9(\mathrm{CH}), 130.8(\mathrm{CH}), 130.5(\mathrm{CH}), 123.1(\mathrm{C}), 122.8(\mathrm{C}), 122.7(\mathrm{C}), 121.1(\mathrm{C}), 120.2(\mathrm{C}), 112.6$ $(\mathrm{CH}), 104.5(\mathrm{C}), 96.2(\mathrm{C}), 91.2(\mathrm{C}), 90.7(\mathrm{C}), 89.5(\mathrm{C}), 88.9(\mathrm{C}), 64.8(\mathrm{C}), 62.8(\mathrm{C}), 55.0\left(\mathrm{CH}_{3}\right), 0.21$ (TMS). MS (MALDI-TOF, m/z): $2113\left(\mathrm{M}^{+}\right), 2007\left(\mathrm{M}^{+}-\mathrm{C}_{7} \mathrm{H}_{7} \mathrm{O}\right), 1841\left(\mathrm{M}^{+}-\mathrm{C}_{19} \mathrm{H}_{17} \mathrm{Si}\right), 1504\left(\mathrm{M}^{+}-\mathrm{C}_{44} \mathrm{H}_{33} \mathrm{O}_{3}\right)$.

Dendron 9. To a solution of $8(50 \mathrm{mg}, 0.024 \mathrm{mmol})$ in $\mathrm{CH}_{2} \mathrm{Cl}_{2}(5 \mathrm{~mL})$ and $\mathrm{MeOH}(2 \mathrm{~mL})$ was added $\mathrm{KOH}(14 \mathrm{mg}, 0.24 \mathrm{mmol})$ under argon and the mixture was stirred at $\mathrm{rt}$ during $1 \mathrm{~h}$. The mixture was concentrated and the residue was dissolved in $\mathrm{CH}_{2} \mathrm{Cl}_{2}(20 \mathrm{~mL})$. The resulting solution was washed with brine $(3 \times 30 \mathrm{~mL})$, dried and concentrated to give a residue that was dissolved in $\mathrm{CH}_{2} \mathrm{Cl}_{2}$. Precipitation with hexanes gave 9 as a white solid [45 mg, $0.022 \mathrm{mmol}, 92 \%, \mathrm{Rf}=0.6\left(30 \%\right.$ toluene $\left.\left./ \mathrm{CH}_{2} \mathrm{Cl}_{2}\right)\right]$. This dendron was immediately used in next reaction. ${ }^{1} \mathrm{H} \mathrm{NMR}\left(\mathrm{CDCl}_{3}, 250 \mathrm{MHz}\right): 7.55-7.36(30 \mathrm{H}, \mathrm{m}, \mathrm{Ar}), 7.21(14 \mathrm{H}$, m, Ar), $7.09\left(18 \mathrm{H}, \mathrm{d}, J=8.7 \mathrm{~Hz}, \mathrm{Ar}_{\mathrm{OMe}}\right), 6.80\left(18 \mathrm{H}, \mathrm{d}, J=8.7 \mathrm{~Hz}, \mathrm{Ar}_{\mathrm{OMe}}\right), 3.80(27 \mathrm{H}, \mathrm{s}, \mathrm{OMe}), 3.18(1 \mathrm{H}, \mathrm{s}$, $\mathrm{C} \equiv \mathrm{C}-\mathrm{H}) .{ }^{13} \mathrm{C} \mathrm{NMR}\left(\mathrm{CDCl}_{3}, 63 \mathrm{MHz}\right): 157.4(\mathrm{C}), 148.1$ (C), 145.9 (C), 138.9 (C), 131.9 (CH), 131.8 $(\mathrm{CH}), 131.4(\mathrm{CH}), 131.0(\mathrm{CH}), 130.9(\mathrm{CH}), 130.8(\mathrm{CH}), 130.5(\mathrm{CH}), 123.9(\mathrm{C}), 123.1(\mathrm{C}), 122.7(\mathrm{C})$, $121.8(\mathrm{C}), 121.0(\mathrm{C}), 120.92(\mathrm{C}), 120.2(\mathrm{C}), 112.6(\mathrm{CH}), 91.2(\mathrm{C}), 90.8(\mathrm{C}), 90.6(\mathrm{C}), 89.5(\mathrm{C}), 89.2(\mathrm{C})$, $88.9(\mathrm{C}), 83.1(\mathrm{C}), 78.9(\mathrm{C}), 64.8(\mathrm{C}), 62.7(\mathrm{C}), 55.0\left(\mathrm{CH}_{3}\right)$. 
Dendrimer G2b. A Schlenk tube equipped with a stirring bar was charged with freshly prepared 9 (340 mg, $0.17 \mathrm{mmol}), 5$ (33 mg, $0.037 \mathrm{mmol})$, CuI (1 mg, $0.0074 \mathrm{mmol}), \mathrm{Ph}_{3} \mathrm{P}(2 \mathrm{mg}, 0.0074 \mathrm{mmol})$ and $\left(\mathrm{Ph}_{3} \mathrm{P}\right)_{4} \mathrm{Pd}(12 \mathrm{mg}, 0.015 \mathrm{mmol})$. After vacuum-drying, activated $4 \AA$ molecular sieves and piperidine $(20$ $\mathrm{mL}$ ) were added under argon. The Schlenk tube was sealed with a teflon screw cap and the reaction mixture was stirred at $100{ }^{\circ} \mathrm{C}$ during $12 \mathrm{~h}$. Then it was allowed to reach $\mathrm{rt}$. A white precipitate was formed, which was filtered and washed with refluxing $\mathrm{CH}_{2} \mathrm{Cl}_{2} /$ hexanes and EtOAc/hexanes to give G2b as a poorly soluble white solid $\left(200 \mathrm{mg}, 0.024 \mathrm{mmol}, 64 \%, \mathrm{Rf}=0.54\left(20 \%\right.\right.$ toluene/ $\left.\mathrm{CH}_{2} \mathrm{Cl}_{2}\right) .{ }^{1} \mathrm{H} \mathrm{NMR}\left(\mathrm{CDCl}_{3}\right.$, $250 \mathrm{MHz}):$ 7.54-7.36 (128H, m, Ar), $7.19(64 \mathrm{H}, \mathrm{m}, \mathrm{Ar}), 7.08\left(72 \mathrm{H}, \mathrm{d}, \mathrm{J}=7.4 \mathrm{~Hz}, \mathrm{Ar}_{\text {OMe }}\right), 6.79$ (72H, d, $\left.J=7.4 \mathrm{~Hz} \mathrm{Ar}_{\mathrm{OMe}}\right), 3.79(108 \mathrm{H}, \mathrm{s}, \mathrm{OMe}) .{ }^{13} \mathrm{C} \mathrm{NMR}\left(\mathrm{CDCl}_{3}, 63 \mathrm{MHz}\right): 157.4(\mathrm{C}), 148.1(\mathrm{C}), 145.9(\mathrm{C}), 138.8$ (C), $131.8(\mathrm{CH}), 131.4(\mathrm{CH}), 131.0(\mathrm{CH}), 130.9(\mathrm{CH}), 130.7(\mathrm{CH}), 130.5(\mathrm{CH}), 123.1(\mathrm{C}), 122.9(\mathrm{C})$, $122.7(\mathrm{C}), 121.0(\mathrm{C}), 120.2(\mathrm{C}), 112.6(\mathrm{CH}), 91.2(\mathrm{C}), 90.9(\mathrm{C}), 90.7(\mathrm{C}), 90.6(\mathrm{C}), 89.5(\mathrm{C}), 88.9(\mathrm{C})$ $64.6(\mathrm{C}), 62.7(\mathrm{C}), 55.0\left(\mathrm{CH}_{3}\right)$. MS (MALDI-TOF, m/z): $17074\left(\mathrm{M}_{2} \mathrm{Ag}^{+}\right), 8591\left(\mathrm{MAg}^{+}\right), 8375\left(\mathrm{M}^{+}-\right.$ $\left.\mathrm{C}_{7} \mathrm{H}_{7} \mathrm{O}\right), 7872\left(\mathrm{M}^{+}-\mathrm{C}_{44} \mathrm{H}_{33} \mathrm{O}_{3}\right)$.

Protected dendron 12 from 11. A Schlenk tube equipped with a stirring bar was charged with $\mathbf{1 1}^{11 \mathrm{~b}}$ (190 mg, $0.24 \mathrm{mmol}), 6^{14}$ (70 mg, $\left.0.36 \mathrm{mmol}\right), \mathrm{CuI}(4 \mathrm{mg}, 0.047 \mathrm{mmol}), \mathrm{Ph}_{3} \mathrm{P}(12 \mathrm{mg}, 0.024 \mathrm{mmol})$ and $\left(\mathrm{Ph}_{3} \mathrm{P}\right)_{4} \mathrm{Pd}(27 \mathrm{mg}, 0.024 \mathrm{mmol})$. After vacuum-drying, activated $4 \AA$ molecular sieves and piperidine $(15$ $\mathrm{mL}$ ) were added under argon. The Schlenk tube was sealed with a teflon screw cap and the reaction mixture was stirred at $100{ }^{\circ} \mathrm{C}$ during $12 \mathrm{~h}$ and then it was allowed to reach $\mathrm{rt}$. The mixture was concentrated and the residue was dissolved in $\mathrm{CH}_{2} \mathrm{Cl}_{2}(30 \mathrm{~mL})$ and washed with a saturated solution of $\mathrm{NH}_{4} \mathrm{Cl}(3 \times 20 \mathrm{~mL})$. The organic layer was dried, filtered and concentrated. The residue was dissolved in $\mathrm{CH}_{2} \mathrm{Cl}_{2}$, and the product was precipitated with hexanes. The precipitate was filtered and reprecipitated three times to give 12 as a yellow solid [120 mg, $0.14 \mathrm{mmol}, 60 \%$ ]. ${ }^{1} \mathrm{H} \mathrm{NMR}\left(\mathrm{CDCl}_{3}, 250 \mathrm{MHz}\right): 7.41$ (4H, s, Ar), $7.36(2 \mathrm{H}, \mathrm{d}, J=7.5 \mathrm{~Hz}, \mathrm{Ar}), 7.28(6 \mathrm{H}, \mathrm{d}, J=7.5 \mathrm{~Hz}, \mathrm{Ar}), 7.22(2 \mathrm{H}, \mathrm{d}, J=7.5 \mathrm{~Hz}, \mathrm{Ar}), 7.16(6 \mathrm{H}$, d, J=7.5 Hz, Ar), $3.73\left(12 \mathrm{H}, \mathrm{s}, \mathrm{CH}_{2}-\mathrm{N}\right), 1.66\left(18 \mathrm{H}, \mathrm{s}, \mathrm{CH}_{2}\right), 0.23(9 \mathrm{H}, \mathrm{s}, \mathrm{TMS}) .{ }^{13} \mathrm{C}$ NMR $\left(\mathrm{CDCl}_{3}, 63\right.$ MHz): $148.5(\mathrm{C}), 147.8(\mathrm{C}), 143.8(\mathrm{C}), 131.7(\mathrm{CH}), 131.5(\mathrm{CH}), 131.2(\mathrm{CH}), 131.1(\mathrm{CH}), 130.5(\mathrm{CH})$, $123.3(\mathrm{C}), 122.5(\mathrm{C}), 120.1(\mathrm{C}), 119.3(\mathrm{CH}), 104.6(\mathrm{C}), 95.9(\mathrm{C}), 91.3(\mathrm{C}), 88.7(\mathrm{C}), 63.9(\mathrm{C}), 47.9(\mathrm{broad}$, 
$\left.\mathrm{CH}_{2}-\mathrm{N}\right), 25.1\left(\mathrm{CH}_{2}\right), 24.3\left(\mathrm{CH}_{2}\right), 0.00(\mathrm{TMS}) . \mathrm{MS}(\mathrm{EI}, \mathrm{m} / \mathrm{z}, \%): 849.2\left(\mathrm{M}^{+}, 7\right), 737.9\left(\mathrm{M}^{+}-\mathrm{C}_{5} \mathrm{H}_{10} \mathrm{~N}_{3}, 15\right)$,

$514.9\left[\mathrm{M}^{+}-3 \mathrm{x}\left(\mathrm{C}_{5} \mathrm{H}_{10} \mathrm{~N}_{3}\right), 100\right], 438.9$ (68). HRMS (EI) calcd. for $\mathrm{C}_{53} \mathrm{H}_{59} \mathrm{~N}_{9} \mathrm{Si}\left(\mathrm{M}^{+}\right)$849.4663, found 849.4655.

Protected dendron 12 from 10. A Schlenk tube equipped with a stirring bar was charged with $\mathbf{1 0}^{11 \mathrm{~b}}$ (88 mg, $0.13 \mathrm{mmol}), 2^{12}(66 \mathrm{mg}, 0.26 \mathrm{mmol}), \mathrm{CuI}(3 \mathrm{mg}, 0.014 \mathrm{mmol}), \mathrm{Ph}_{3} \mathrm{P}(7 \mathrm{mg}, 0.026 \mathrm{mmol})$ and $\left(\mathrm{Ph}_{3} \mathrm{P}\right)_{4} \mathrm{Pd}(15 \mathrm{mg}, 0.014 \mathrm{mmol})$. After vacuum-drying, activated $4 \AA$ molecular sieves and piperidine $(15$ $\mathrm{mL}$ ) were added under argon. The Schlenk tube was sealed with a teflon screw cap and the reaction mixture was stirred at $100{ }^{\circ} \mathrm{C}$ during $12 \mathrm{~h}$ and then it was allowed to reach $\mathrm{rt}$. The mixture was concentrated and the residue was dissolved in $\mathrm{CH}_{2} \mathrm{Cl}_{2}(20 \mathrm{~mL})$ and washed with a saturated solution of $\mathrm{NH}_{4} \mathrm{Cl}(3 \times 10 \mathrm{~mL})$. The organic layer was dried, filtered and concentrated. The residue was dissolved in $\mathrm{CH}_{2} \mathrm{Cl}_{2}$, and the product was precipitated with hexanes. The precipitate was filtered and reprecipitated three times to give $\mathbf{1 2}$ as a yellow solid [90 mg, $0.11 \mathrm{mmol}, 81 \%$ ]. Its spectroscopical data were identical to those of the product obtained from the previous experiment.

Dendron 13. To a solution of $12(268 \mathrm{mg}, 0.32 \mathrm{mmol})$ in $\mathrm{CH}_{2} \mathrm{Cl}_{2}(15 \mathrm{~mL})$ and $\mathrm{MeOH}(2 \mathrm{~mL})$ was added $\mathrm{KOH}(177 \mathrm{mg}, 3.15 \mathrm{mmol}$ ) under argon and the mixture was stirred at $\mathrm{rt}$ during $1 \mathrm{~h}$. The mixture was concentrated and the residue was dissolved in $\mathrm{CH}_{2} \mathrm{Cl}_{2}(30 \mathrm{~mL})$ and the solution was washed with brine (3x20 mL), dried and concentrated. The resulting residue was dissolved in $\mathrm{CH}_{2} \mathrm{Cl}_{2}$ followed by precipitation with hexanes to give $\mathbf{1 3}$ as a brown solid [240 $\mathrm{mg}, 0.31 \mathrm{mmol}, 98 \%, \mathrm{Rf}=0.46(20 \%$ EtOAc/hexanes)]. This dendron was immediately used in next reaction. ${ }^{1} \mathrm{H}$ NMR $\left(\mathrm{CDCl}_{3}, 250 \mathrm{MHz}\right)$ : 7.50-7.11 (20H, m, Ar), $3.67\left(12 \mathrm{H}, \mathrm{s}, \mathrm{CH}_{2}-\mathrm{N}\right), 3.16(1 \mathrm{H}, \mathrm{s}, \mathrm{C} \equiv \mathrm{C}-\mathrm{H}), 1.66\left(18 \mathrm{H}, \mathrm{s}, \mathrm{CH}_{2}\right) .{ }^{13} \mathrm{C} \mathrm{NMR}$ $\left(\mathrm{CDCl}_{3}, 63 \mathrm{MHz}\right)$ : $148.6(\mathrm{C}), 147.9(\mathrm{C}), 143.8(\mathrm{C}), 131.9(\mathrm{CH}), 131.5(\mathrm{CH}), 131.3(\mathrm{CH}), 131.1(\mathrm{CH})$, 130.6 (CH), $123.8(\mathrm{C}), 121.6(\mathrm{C}), 120.0(\mathrm{C}), 119.4(\mathrm{CH}), 91.4(\mathrm{C}), 88.6(\mathrm{C}), 83.2(\mathrm{C}), 78.8(\mathrm{CH}), 63.9$ (C), $48.1\left(\right.$ broad, $\left.\mathrm{CH}_{2}-\mathrm{N}\right), 25.1\left(\mathrm{CH}_{2}\right), 24.3\left(\mathrm{CH}_{2}\right)$.

Dendrimer G1b-N3. A Schlenk tube equipped with a stirring bar was charged with freshly prepared 13 (240 mg, $0.31 \mathrm{mmol}), 5^{11,13}(56 \mathrm{mg}, 0.068 \mathrm{mmol}), \mathrm{CuI}(5 \mathrm{mg}, 0.027 \mathrm{mmol}), \mathrm{Ph}_{3} \mathrm{P}(11 \mathrm{mg}, 0.041 \mathrm{mmol})$ 
and $\left(\mathrm{Ph}_{3} \mathrm{P}\right)_{4} \mathrm{Pd}(32 \mathrm{mg}, 0.027 \mathrm{mmol})$ and then the mixture was dried under vacuum. Activated $4 \AA$ molecular sieves and piperidine $(20 \mathrm{~mL})$ were added under argon and the Schlenk tube was sealed with a teflon screw cap. The reaction mixture was stirred at $100{ }^{\circ} \mathrm{C}$ during $12 \mathrm{~h}$ and then it was allowed to reach rt. The mixture was concentrated and the residue was dissolved in $\mathrm{CH}_{2} \mathrm{Cl}_{2}(30 \mathrm{~mL})$ and the solution was washed with a solution of $\mathrm{NH}_{4} \mathrm{Cl}(3 \times 10 \mathrm{~mL})$. The organic phase was dried and concentrated and the residue was purified by column GPC (Bio-Beads S-X1, THF) and precipitation from THF. The precipitate was filtered and washed with refluxing hexanes and then with refluxing $\mathrm{MeOH}$ to give $\mathbf{G 1 b}-\mathbf{N 3}$ as a brown solid (150 mg, $0.044 \mathrm{mmol}, 64 \%) .{ }^{1} \mathrm{H} \mathrm{NMR}\left(\mathrm{CDCl}_{3}, 400 \mathrm{MHz}\right): 7.60-7.00$ (96H, m, Ar), 3.75 (48H, s, $\left.\mathrm{CH}_{2}-\mathrm{N}\right), 1.69\left(72 \mathrm{H}, \mathrm{s}, \mathrm{CH}_{2}\right) \cdot{ }^{13} \mathrm{C} \mathrm{NMR}\left(\mathrm{CDCl}_{3}, 63 \mathrm{MHz}\right): 148.5(\mathrm{C}), 147.9(\mathrm{C}), 144.1(\mathrm{CH}), 143.8(\mathrm{CH})$, $131.5(\mathrm{CH}), 131.0(\mathrm{CH}), 130.5(\mathrm{CH}), 119.3(\mathrm{CH}), 63.9(\mathrm{C}), 48.0\left(\operatorname{broad}, \mathrm{CH}_{2}-\mathrm{N}\right), 25.1\left(\mathrm{CH}_{2}\right), 24.3\left(\mathrm{CH}_{2}\right)$.

Dendrimer G1b-I. A Schlenk tube equipped with a stirring bar was charged with G1b-N3 (70 mg, $0.020 \mathrm{mmol})$ and it was dried under vacuum. Then, MeI $(10 \mathrm{~mL})$ was slowly added under argon, the Schlenk tube was sealed with a teflon screw cap and the reaction mixture was stirred at $120^{\circ} \mathrm{C}$ during 48 h. After reaching $\mathrm{rt}$, the mixture was diluted with $\mathrm{CH}_{2} \mathrm{Cl}_{2}(50 \mathrm{~mL})$ and the resulting solution was washed with saturated solutions of $\mathrm{Na}_{2} \mathrm{~S}_{2} \mathrm{O}_{3}(3 \times 10 \mathrm{~mL})$ and $\mathrm{NH}_{4} \mathrm{Cl}(3 \times 10 \mathrm{~mL})$. The organic phase was dried and concentrated and the poorly soluble residue was washed with refluxing hexanes and then with $\mathrm{MeOH}$ to give G1b-I as a yellow solid (70 mg, $0.019 \mathrm{mmol}, 95 \%) .{ }^{1} \mathrm{H} \mathrm{NMR}\left(\mathrm{CDCl}_{3}, 250 \mathrm{MHz}\right): 8.0-7.33(64 \mathrm{H}$, m, Ar/Ar $)), 7.13(8 \mathrm{H}, \mathrm{d}, J=7.3 \mathrm{~Hz}, \mathrm{Ar}), 6.90\left(24 \mathrm{H}, \mathrm{d}, J=7.9 \mathrm{~Hz}, \mathrm{Ar}_{\mathrm{I}}\right) .{ }^{13} \mathrm{C} \mathrm{NMR}\left(\mathrm{CDCl}_{3}, 63 \mathrm{MHz}\right): 145.0$ (C), $136.9(\mathrm{CH}), 132.5(\mathrm{CH}), 131.8(\mathrm{CH}), 131.4(\mathrm{CH}), 131.0(\mathrm{CH}), 130.9(\mathrm{CH}), 130.5(\mathrm{CH}), 129.6(\mathrm{CH})$, $92.3(\mathrm{C}), 64.4(\mathrm{C}), 63.7$ (C). MS (MALDI-TOF, m/z): $2980\left(\mathrm{M}^{+}-\mathrm{C}_{19} \mathrm{H}_{12} \mathrm{I}_{3}\right), 2361\left[\mathrm{M}^{+}-2 \mathrm{x}\left(\mathrm{C}_{19} \mathrm{H}_{12} \mathrm{I}_{3}\right)\right], 1741$ $\left[\mathrm{M}^{+}-3 \mathrm{x}\left(\mathrm{C}_{19} \mathrm{H}_{12} \mathrm{I}_{3}\right)\right]$.

Dendrimer G1c. Piperidine $(0.2 \mathrm{~mL}, 2.3 \mathrm{mmol})$ was added to a solution $\mathbf{1}^{11}(100 \mathrm{mg}, 0.23 \mathrm{mmol}), \mathrm{CuI}$ $(10 \mathrm{mg}, 0.46 \mathrm{mmol})$ and $\left(\mathrm{Ph}_{3} \mathrm{P}\right)_{4} \mathrm{Pd}(5 \mathrm{mg}, 0.0046 \mathrm{mmol})$ in $\mathrm{THF}(10 \mathrm{~mL})$ and the mixture was stirred at $\mathrm{rt}$ for $2 \mathrm{~h}$ in an open round bottom flask. The mixture was concentrated, the residue was dissolved in EtOAc $(20 \mathrm{~mL})$ and was washed with a solution of $\mathrm{NH}_{4} \mathrm{Cl}(3 \times 15 \mathrm{~mL})$. The organic phase was dried and concentrated and the residue was purified by flash chromatography $\left(\mathrm{SiO}_{2}, 15 \% \mathrm{EtOAc} /\right.$ hexanes $)$ to give 
G1c as a white solid [98 mg, $0.075 \mathrm{mmol}, 98 \%, \mathrm{Rf}=0.4$ (30\% EtOAc/hexanes)]. ${ }^{1} \mathrm{H} \mathrm{NMR}\left(\mathrm{CDCl}_{3}, 250\right.$ MHz): 7.40 (4H, d, J=8.4 Hz, Ar), $7.18(4 \mathrm{H}, \mathrm{d}, J=8.4 \mathrm{~Hz}, \mathrm{Ar}), 7.07\left(12 \mathrm{H}, \mathrm{d}, J=8.9 \mathrm{~Hz}, \mathrm{Ar}_{\mathrm{OMe}}\right), 6.79(12 \mathrm{H}$ d, J=8.9 Hz $\left.\mathrm{Ar}_{\text {Оме }}\right), 3.79$ (18H, s, OMe). ${ }^{13} \mathrm{C} \mathrm{NMR}\left(\mathrm{CDCl}_{3}, 63 \mathrm{MHz}\right): 157.7$ (C), 149.2 (C), 138.9 (C), 132.1 (CH), $131.7(\mathrm{CH}), 131.1(\mathrm{CH}), 119.2(\mathrm{C}), 112.9(\mathrm{CH}), 81.7(\mathrm{C}), 74.0(\mathrm{C}), 63.0(\mathrm{C}), 55.3\left(\mathrm{CH}_{3}\right) . \mathbf{M S}$ (EI, m/z, \%): $866\left(\mathrm{M}^{+}, 40\right), 759\left(\mathrm{M}^{+}-\mathrm{C}_{7} \mathrm{H}_{7} \mathrm{O}, 39\right), 333\left(\mathrm{C}_{22} \mathrm{H}_{21} \mathrm{O}_{3}{ }^{+}, 100\right) . \mathrm{MS}(\mathrm{EI}, \mathrm{m} / \mathrm{z}, \%): 893.2\left(\mathrm{M}^{+}, 100\right)$, 690.4 (M+- $\left.\mathrm{M}_{6} \mathrm{H}_{5} \mathrm{I}, 67\right)$. HRMS (EI) calcd. For $\mathrm{C}_{60} \mathrm{H}_{50} \mathrm{O}_{6}{ }^{+}\left(\mathrm{M}^{+}\right)$866.3607, found 866.3607.

Compound 15. A Schlenk tube equipped with a stirring bar was charged with freshly prepared $\mathbf{1}^{11}(250$ $\mathrm{mg}, 0.58 \mathrm{mmol}), \mathbf{1 4}^{6}(118 \mathrm{mg}, 0.17 \mathrm{mmol}), \mathrm{CuI}(6 \mathrm{mg}, 0.033 \mathrm{mmol}), \mathrm{Ph}_{3} \mathrm{P}(9 \mathrm{mg}, 0.033 \mathrm{mmol})$ y $\left(\mathrm{Ph}_{3} \mathrm{P}\right)_{4} \mathrm{Pd}$ (57 mg, $0.050 \mathrm{mmol})$ and then the mixture was dried under vacuum. Activated $4 \AA$ molecular sieves and piperidine $(20 \mathrm{~mL})$ were added under argon and the Schlenk tube was sealed with a teflon screw cap. The reaction mixture was stirred at $100{ }^{\circ} \mathrm{C}$ during $12 \mathrm{~h}$ and then it was allowed to reach $\mathrm{rt}$. The mixture was concentrated and the residue was dissolved in $\mathrm{CH}_{2} \mathrm{Cl}_{2}(20 \mathrm{~mL})$ and the solution was washed with a solution of $\mathrm{NH}_{4} \mathrm{Cl}(3 \times 15 \mathrm{~mL})$. The organic phase was dried and concentrated and the residue was purified by flash chromatography $\left(\mathrm{SiO}_{2}, 30 \%\right.$ EtOAc/hexanes) to give 15 as a yellow solid [196 mg, $0.12 \mathrm{mmol}, 73 \%$, $\mathrm{Rf}=0.42(40 \% \mathrm{EtOAc} / \mathrm{hexanes})] .{ }^{1} \mathrm{H} \mathrm{NMR}\left(\mathrm{CDCl}_{3}, 250 \mathrm{MHz}\right): 7.38(12 \mathrm{H}, \mathrm{m}, \mathrm{Ar}), 7.19(12 \mathrm{H}, \mathrm{d}, J=8.3$ $\mathrm{Hz}, \mathrm{Ar}), 7.10\left(22 \mathrm{H}, \mathrm{m}, \mathrm{Ar}_{\mathrm{OMe} / \mathrm{OH}}\right), 6.80\left(20 \mathrm{H}, \mathrm{d}, \mathrm{J}=8.7 \mathrm{~Hz}, \mathrm{Ar}_{\mathrm{OMe}}\right), 6.74\left(2 \mathrm{H}, \mathrm{d}, J=9.1 \mathrm{~Hz}, \mathrm{Ar}_{\mathrm{OH}}\right) 3.80(27 \mathrm{H}$, s, OMe $) .{ }^{13} \mathrm{C} \mathrm{NMR}\left(\mathrm{CDCl}_{3}, 63 \mathrm{MHz}\right): 157.4(\mathrm{C}), 147.9(\mathrm{C}), 146.4(\mathrm{C}), 138.9(\mathrm{C}), 132.0(\mathrm{CH}), 131.9(\mathrm{CH})$, $131.1(\mathrm{CH}), 130.8(\mathrm{CH}), 130.5(\mathrm{CH}), 121.0(\mathrm{C}), 120.4(\mathrm{C}), 112.6(\mathrm{CH}), 89.4(\mathrm{C}), 88.9(\mathrm{C}), 62.6(\mathrm{C}), 55.1$ $\left(\mathrm{CH}_{3}\right)$. MS (MALDI-TOF, m/z): $1632.6\left(\mathrm{M}^{+}\right)$.

Compound 16. A solution of 15 (120 mg, $0.073 \mathrm{mmol})$ and $\mathrm{Et}_{3} \mathrm{~N}(0.036 \mathrm{~mL}, 0.256 \mathrm{mmol})$ in $\mathrm{dry}_{\mathrm{CH}_{2}} \mathrm{Cl}_{2}$ $(20 \mathrm{~mL})$ was cooled to $-78{ }^{\circ} \mathrm{C}$ during $10 \mathrm{~min}$. Then, $\mathrm{Tf}_{2} \mathrm{O}(0.024 \mathrm{~mL}, 0.147 \mathrm{mmol})$ was added and the mixture was allowed to reach $\mathrm{rt}$ for $12 \mathrm{~h}$. The mixture was washed with a saturated solution of $\mathrm{NH}_{4} \mathrm{Cl}(40$ $\mathrm{mL})$ and the aqueous phase was extracted with EtOAc $(3 \times 30 \mathrm{~mL})$. The combined organic phase was dried and concentrated and the residue was purified by flash chromatography $\left(\mathrm{SiO}_{2}, 20 \% \mathrm{EtOAc} /\right.$ hexanes$)$ to give 16 as a white solid [122 mg, $0.069 \mathrm{mmol}, 95 \%, \mathrm{Rf}=0.65$ (40\% EtOAc/hexanes)]. ${ }^{1} \mathrm{H} \mathrm{NMR}\left(\mathrm{CDCl}_{3}\right.$, $250 \mathrm{MHz}):$ 7.46-7.38 (12H, m, Ar), $7.30\left(2 \mathrm{H}, \mathrm{d}, \mathrm{J}=8.9 \mathrm{~Hz}, \mathrm{Ar}_{\mathrm{OTf}}\right), 7.25-7.10(14 \mathrm{H}, \mathrm{m}, \mathrm{Ar}), 7.07$ (18H, d, 
$\left.\mathrm{d}, J=8.9 \mathrm{~Hz}, \mathrm{Ar}_{\mathrm{OMe}}\right), 6.77\left(18 \mathrm{H}, \mathrm{d}, J=8.9 \mathrm{~Hz}, \mathrm{Ar}_{\mathrm{OMe}}\right), 3.75(27 \mathrm{H}, \mathrm{s}, \mathrm{OMe}) .{ }^{13} \mathrm{C} \mathrm{NMR}\left(\mathrm{CDCl}_{3}, 63 \mathrm{MHz}\right)$ :

$157.4(\mathrm{C}), 148.0(\mathrm{C}), 145.3(\mathrm{C}), 138.9(\mathrm{C}), 132.5(\mathrm{CH}), 131.8(\mathrm{CH}), 131.1(\mathrm{CH}), 130.8(\mathrm{CH}), 130.5(\mathrm{CH})$, $121.5(\mathrm{C}), 120.5(\mathrm{CH}), 120.2(\mathrm{C}), 112.6(\mathrm{CH}), 89.8(\mathrm{C}), 88.5(\mathrm{C}), 62.8(\mathrm{C}), 55.0\left(\mathrm{CH}_{3}\right)$. MS (MALDITOF, m/z): $1764.5\left(\mathrm{M}^{+}\right)$.

Protected dendron 17. A Schlenk tube equipped with a stirring bar was charged with $\mathbf{1 6}$ (116 g, 0.066 mmol), $\mathrm{CuI}(1 \mathrm{mg}, 0.0066 \mathrm{mmol}), \mathrm{Ph}_{3} \mathrm{P}(3 \mathrm{mg}, 0.0131 \mathrm{mmol})$ y $\left(\mathrm{Ph}_{3} \mathrm{P}\right)_{4} \mathrm{Pd}(8 \mathrm{mg}, 0.0066 \mathrm{mmol})$. After vacuum-drying, activated $4 \AA$ molecular sieves, trimethylsilylacetilene $(0.056 \mathrm{~mL}, 0.39 \mathrm{mmol})$ and piperidine $(15 \mathrm{~mL})$ were successively added under argon. The Schlenk tube was sealed with a teflon screw cap and the reaction mixture was stirred at $100{ }^{\circ} \mathrm{C}$ during $12 \mathrm{~h}$ and then it was allowed to reach rt. The mixture was concentrated and the residue was dissolved in $\mathrm{CH}_{2} \mathrm{Cl}_{2}(20 \mathrm{~mL})$ and washed with a saturated solution of $\mathrm{NH}_{4} \mathrm{Cl}(3 \times 10 \mathrm{~mL})$. The organic layer was dried, filtered and concentrated and the residue was purified by flash chromatography $\left(\mathrm{SiO}_{2}, 20 \% \mathrm{CH}_{2} \mathrm{Cl}_{2} /\right.$ hexanes$)$ to give $17^{11 \mathrm{a}}$ as a white solid [107 $\mathrm{mg}$, $0.062 \mathrm{mmol}, 95 \%, \mathrm{Rf}=0.42(30 \% \mathrm{EtOAc} / \mathrm{hexanes})]$. Its spectroscopic data were identical to those of a reference sample.

Dendrimer G2c. Piperidine (0.04 mL, $0.47 \mathrm{mmol})$ was added to a solution of $18(153 \mathrm{mg}, 0.093 \mathrm{mmol}$ prepared from 17 as previously described by our group $\left.{ }^{11 \mathrm{a}}\right), \mathrm{CuI}(3 \mathrm{mg}, 0.19 \mathrm{mmol})$ and $\left(\mathrm{Ph}_{3} \mathrm{P}\right)_{4} \mathrm{Pd}(22 \mathrm{mg}$, $0.019 \mathrm{mmol})$ in THF $(10 \mathrm{~mL})$ and the mixture was stirred at $\mathrm{rt}$ for $2 \mathrm{~h}$ in an open round bottom flask. The mixture was concentrated, the residue was dissolved in EtOAc $(20 \mathrm{~mL})$ and was washed with a solution of $\mathrm{NH}_{4} \mathrm{Cl}(3 \times 15 \mathrm{~mL})$. The organic phase was dried and concentrated and the residue was purified by flash chromatography $\left(\mathrm{SiO}_{2}, 25 \%\right.$ EtOAc/hexanes) to give G2c as a white solid [150 mg, $0.046 \mathrm{mmol}, 98 \%$, $\mathrm{Rf}=0.45\left(80 \% \mathrm{CH}_{2} \mathrm{Cl}_{2} /\right.$ hexanes $\left.)\right] .{ }^{1} \mathrm{H} \mathrm{NMR}\left(\mathrm{CDCl}_{3}, 250 \mathrm{MHz}\right): 7.42(28 \mathrm{H}, \mathrm{m}, \mathrm{Ar}), 7.19(28 \mathrm{H}, \mathrm{d}, \mathrm{J}=8.1$ $\mathrm{Hz}, \mathrm{Ar}), 7.10\left(36 \mathrm{H}, \mathrm{d}, J=8.8 \mathrm{~Hz}, \mathrm{Ar}_{\mathrm{OMe}}\right), 6.80\left(36 \mathrm{H}, \mathrm{d}, J=8.8 \mathrm{~Hz} \mathrm{Ar}_{\mathrm{OMe}}\right), 3.80(54 \mathrm{H}, \mathrm{s}, \mathrm{OMe}) .{ }^{13} \mathrm{C}$ NMR $\left(\mathrm{CDCl}_{3}, 63 \mathrm{MHz}\right): 157.6(\mathrm{C}), 148.2(\mathrm{C}), 147.2(\mathrm{C}), 145.7(\mathrm{C}), 139.17(\mathrm{C}), 132.1(\mathrm{CH}), 131.2(\mathrm{CH}), 131.0$ CH), $130.9(\mathrm{CH}), 130.8(\mathrm{CH}), 121.6(\mathrm{C}), 120.6(\mathrm{C}), 119.9(\mathrm{C}), 112.9(\mathrm{CH}), 89.9(\mathrm{C}), 88.9(\mathrm{C}), 81.5(\mathrm{C})$, $74.4(\mathrm{C}), 65.0(\mathrm{C}), 62.7(\mathrm{C}), 55.3\left(\mathrm{CH}_{3}\right)$. MS (MALDI-TOF, m/z): $3389\left(\mathrm{MAg}^{+}\right), 3280\left(\mathrm{M}^{+}\right), 3174\left(\mathrm{M}^{+}-\right.$ $\left.\mathrm{C}_{7} \mathrm{H}_{7} \mathrm{O}\right), 2772\left(\mathrm{M}^{+}-\mathrm{C}_{36} \mathrm{H}_{29} \mathrm{O}_{3}\right), 1540\left(\mathrm{M}^{+}-\mathrm{C}_{109} \mathrm{H}_{87} \mathrm{O}_{9}\right)$. 
Dendrimer G1c-N3. Piperidine $(0.110 \mathrm{~mL}, 1.3 \mathrm{mmol})$ was added to a solution of $\mathbf{1 0}^{11 \mathrm{~b}}(88 \mathrm{mg}, 0.13$ $\mathrm{mmol}), \mathrm{CuI}(50 \mathrm{mg}, 0.26 \mathrm{mmol})$ and $\left(\mathrm{Ph}_{3} \mathrm{P}\right)_{4} \mathrm{Pd}(15 \mathrm{mg}, 0.013 \mathrm{mmol})$ in THF $(10 \mathrm{~mL})$ and the mixture was stirred at $\mathrm{rt}$ for $2 \mathrm{~h}$ in an open round bottom flask. The mixture was concentrated, the residue was dissolved in $\mathrm{CH}_{2} \mathrm{Cl}_{2}(10 \mathrm{~mL})$ and was washed with a solution of $\mathrm{NH}_{4} \mathrm{Cl}(3 \times 10 \mathrm{~mL})$. The organic phase was dried and diluted with hexanes until precipitation of a solid. The precipitate was filtered and dried to give G1c-N3 (85 mg, $0.063 \mathrm{mmol}, 97 \%) .{ }^{1} \mathrm{H} \mathrm{NMR}\left(\mathrm{CDCl}_{3}, 250 \mathrm{MHz}\right): 7.35$ (4H, d, J=8.0 Hz, Ar), $7.27(12 \mathrm{H}$, d, J=8.3 Hz, Ar), $7.20(4 \mathrm{H}, \mathrm{d}, J=8.0 \mathrm{~Hz}, \mathrm{Ar}), 7.14(12 \mathrm{H}, \mathrm{d}, J=8.3 \mathrm{~Hz}, \mathrm{Ar}), 3.72\left(24 \mathrm{H}, \mathrm{s}, \mathrm{CH}_{2}-\mathrm{N}\right), 1.65$ (36H, s, $\left.\mathrm{CH}_{2}\right) .{ }^{13} \mathrm{C} \mathrm{NMR}\left(\mathrm{CDCl}_{3}, 63 \mathrm{MHz}\right): 148.6(\mathrm{C}), 143.6(\mathrm{C}), 131.4(\mathrm{CH}), 131.0(\mathrm{CH}), 119.4(\mathrm{CH})$, $119.0(\mathrm{C}), 81.4(\mathrm{C}), 73.8(\mathrm{C}), 64.0(\mathrm{C}), 48.0\left(\mathrm{broad}, \mathrm{CH}_{2}-\mathrm{N}\right), 25.1\left(\mathrm{CH}_{2}\right), 24.2\left(\mathrm{CH}_{2}\right)$.

Dendrimer G1c-I. A Schlenk tube equipped with a stirring bar was charged with G1c-N3 (8 mg, 0.059 mmol) and it was dried under vacuum. Then, MeI (5 mL) was slowly added under argon, the Schlenk tube was sealed with a teflon screw cap and the reaction mixture was stirred at $120^{\circ} \mathrm{C}$ during $48 \mathrm{~h}$. After reaching $\mathrm{rt}$, the mixture was diluted with $\mathrm{CH}_{2} \mathrm{Cl}_{2}(50 \mathrm{~mL})$ and the resulting solution was washed with saturated solutions of $\mathrm{Na}_{2} \mathrm{~S}_{2} \mathrm{O}_{3}(3 \times 10 \mathrm{~mL})$ and $\mathrm{NH}_{4} \mathrm{Cl}(3 \times 10 \mathrm{~mL})$. The organic phase was dried and concentrated and the poorly soluble residue was washed with refluxing hexanes and then with $\mathrm{MeOH}$ to give G1c-I as a yellow solid (70 mg, $0.059 \mathrm{mmol}, 82 \%) .{ }^{1} \mathrm{H} \mathrm{NMR}\left(\mathrm{CDCl}_{3}, 250 \mathrm{MHz}\right): 7.58(12 \mathrm{H}, \mathrm{d}, \mathrm{J}=8.5$ $\left.\mathrm{Hz}, \mathrm{Ar}_{\mathrm{I}}\right), 7.41(4 \mathrm{H}, \mathrm{d}, J=8.3 \mathrm{~Hz}, \mathrm{Ar}), 7.11(4 \mathrm{H}, \mathrm{d}, J=8.5 \mathrm{~Hz}, \mathrm{Ar}), 6.88\left(12 \mathrm{H}, \mathrm{d}, J=8.5 \mathrm{~Hz} \mathrm{Ar}_{\mathrm{I}}\right)$.

Dendrimer G3c. A Schlenk tube equipped with a stirring bar was charged with freshly prepared G1cI (15 mg, $0.010 \mathrm{mmol}), \mathbf{1 8}(152 \mathrm{mg}, 0.093 \mathrm{mmol}$, prepared from $\mathbf{1 7}$ as previously described by our group $\left.{ }^{11 \mathrm{a}}\right), \mathrm{CuI}(2 \mathrm{mg}, 0.0063 \mathrm{mmol}), \mathrm{Ph}_{3} \mathrm{P}(2 \mathrm{mg}, 0.0063 \mathrm{mmol})$ y $\left(\mathrm{Ph}_{3} \mathrm{P}\right)_{4} \mathrm{Pd}(8 \mathrm{mg}, 0.0073 \mathrm{mmol})$. After vacuum-drying, activated $4 \AA$ molecular sieves and piperidine $(15 \mathrm{~mL})$ were added under argon. The Schlenk tube was sealed with a teflon screw cap and the reaction mixture was stirred at $100{ }^{\circ} \mathrm{C}$ during 12 $\mathrm{h}$ and then it was allowed to reach $\mathrm{rt}$. The resulting mixture was concentrated and the residue was dissolved in $\mathrm{CH}_{2} \mathrm{Cl}_{2}(20 \mathrm{~mL})$ and washed with a saturated solution of $\mathrm{NH}_{4} \mathrm{Cl}(3 \times 15 \mathrm{~mL})$. The organic layer was dried and concentrated to give a residue, which was purified by column GPC (Bio-Beads SX-1, THF) and precipitation from THF solution. The precipitate was filtered and washed with refluxing hexanes and 
methanol to give dendrimer G3c as a white solid [88 $\mathrm{mg}, 0.0084 \mathrm{mmol}, 80 \%, \mathrm{Rf}=0.55(10 \%$ hexanes/ $\left.\mathrm{CH}_{2} \mathrm{Cl}_{2}\right)$ ]. ${ }^{1} \mathrm{H}$ NMR $\left(\mathrm{CDCl}_{3}, 250 \mathrm{MHz}\right): 7.41(100 \mathrm{H}, \mathrm{m}, \mathrm{Ar}), 7.19$ (100H, m, CH-Ar), 7.09 (108H, d, J=8.7 $\mathrm{Hz}, \mathrm{Ar}), 6.79\left(108 \mathrm{H}, \mathrm{d}, J=8.7 \mathrm{~Hz}, \mathrm{Ar}_{\mathrm{OMe}}\right), 3.78(162 \mathrm{H}, \mathrm{s}, \mathrm{OMe}) .{ }^{13} \mathrm{C} \mathrm{NMR}\left(\mathrm{CDCl}_{3}, 63 \mathrm{MHz}\right): 157.4(\mathrm{C})$, $147.9(\mathrm{C}), 145.9(\mathrm{C}), 145.7(\mathrm{C}), 145.7(\mathrm{C}), 138.9(\mathrm{C}), 131.8(\mathrm{CH}), 130.9(\mathrm{CH}), 130.8(\mathrm{CH}), 130.7(\mathrm{CH})$, $130.5(\mathrm{CH}), 121.2(\mathrm{C}), 121.0(\mathrm{C}), 120.3(\mathrm{C}), 112.6(\mathrm{CH}), 89.6(\mathrm{C}), 89.3(\mathrm{C}), 89.1(\mathrm{C}), 88.7(\mathrm{C}), 64.7(\mathrm{C})$, 62.7 (C), $55.0\left(\mathrm{CH}_{3}\right)$. MS (MALDI-TOF, m/z): $10636.8\left(\mathrm{MAg}^{+}\right)$.

Acknowledgment. We acknowledge financial support from Spanish MINECO (grant MAT201342425-R) and MICINN (grant PGC2018-101047-B-I00), co-funding from FEDER, and a predoctoral fellowship from the Chile Government to J.I.U. (CONICYT Becas Chile).

Supporting Information Available: Copies of NMR spectra (PDF).

\section{References}

1 (a) S. Sengupta, S. K. Sadhukhan, Tetrahedron Lett. 1998, 39, 1237-1238. (b) N. Armaroli, V. Balzani, J. P. Collin, P. Gavina, J. P. Sauvage, B. Ventura, J. Am. Chem. Soc. 1999, 121, 4397-4408. (c) M. R. Robinson, S. Wang, G. C. Bazan, Y. Cao, Adv. Mater. 2000, 12, 1701-1704. (d) M. Quintiliani, A. Kahnt, P. Vásquez, D. M. Guldi, T. Torres, J. Mater. Chem. 2008, 18, 1542-1546. (e) T. Muller, S. Bräse, RSC Adv. 2014, 4, 6886.

2 (a) B. F. Hoskins, R. Robson, J. Am. Chem. Soc. 1990, 112, 1546-1554. (b) D. Venkataraman, S. Lee, J. S. Moore, P. Zhang, K. A. Hirsch, G. B. Gardner, A. C. Corey, C. L. Prentice, Chem. Mater. 1996, 8, 2030-2040. (c) A. Tanatini, T. S. Hughes, J. S. Moore, Angew. Chem. Int. Ed. Engl. 2002, 41, 325-328. (d) J. H. Ryu, H. J. Kim, Z. Huang, E. Lee, M. Lee, Angew. Chem. Int. Ed. 2006, 45, 5304-5307. (e) M. Quintiliani, A. Kahnt, P. Vásquez, D. M. Guldi, T. Torres, J. Mater. Chem. 2008, 18, 1542-1546. (f) J. Tian, Y. Ding, T. Zhou, K. Zhang, X. Zhao, H. Wang, D. Zhang, Y. Liu, Z. Li, Chem. Eur. J. 2014, 20, $575-584$. 
3 (a) S. Wang, W. J. Jr. Oldham, R. A. Jr. Hudack, G. C. Bazan, J. Am. Chem. Soc. 2000, 122, 5695-

5709. (b) X. M. Liu, C. He, J. Huang, J. W. Xu, Chem. Mater. 2005, 17, 434-441. (c) C. Lee. J. Lee, Chem. Eur. J. 2012, 18, 6457-6461.

4 (a) S. Wang, W. J. Jr. Oldham, R. A. Jr. Hudack, G. C. Bazan, J. Am. Chem. Soc. 2000, 122, 56955709. (b) L. H. Chan, R. H. Lee, C. F. Hsieh, H. C. Yeh, C. T. J. Chen, J. Am. Chem. Soc. 2002, 124, 6469-6479. (c) X. M. Liu, C. He, J. Huang, J. W. Xu, Chem. Mater. 2005, 17, 434-441. (d) K. Tsuchiya, M. Ueda. Polym. J. 2006, 38, 956-960. (e) M. Quintiliani, A. Kahnt, P. Vázquez, D. M. Guldi, T. Torres, J. Mater. Chem. 2008, 18, 1542-1546. (f) C. Lee. J. Lee, Chem. Eur. J. 2012, 18, 6457-6461.

5 (a) A. Pegenau, T. Hegmann, C. Tschierske, S. Diele, Chem. Eur. J. 1999, 5, 1643-1660. (b) T. Hatano, T. Kato, Tetrahedron 2008, 64, 8368-8380.

6 M. E. Gallina, B. Baytekin, C. Schalley, P. Ceroni, Chem. Eur. J. 2012, 18, 1528-1535.

7 (a) U. M. Wiesler, A. J. Berresheim, F. Morgenroth, G. Lieser, K. Mullen, Macromolecules 2001, 34, 187-199. (b) M. Schlupp, T. Weil, A. J. Berresheim, U. M. Wiesler, J. Bargon, K. Müllen, Angew. Chem. Int. Ed. 2001, 40, 4011-4015. (c) B. A. G. Hammer, R. Moritz, R. Stangenberg, M. Baumgarten, K. Müllen, Chem. Soc.Rev. 2015, 44, 4072-4090. (d) M. Brutschy, R. Stangenberg, C. Beer, D. Lubczyk, M. Baumgarten, K. Müllen, S. R. Waldwogel, ChemPlusChem 2015, 80, 54-56.

8 Y. Jin, A. Jin, R. McCaffrey, H. Long, W. Zhang, J. Org. Chem. 2012, 77, 7392-7400.

9 (a) J. Kim, B. Chen, T. M. Reineke, H. Li, M. Eddaoudi, D. B. Moler, M. O’Keeffe, O. M. Yaghi. J. Am. Chem. Soc. 2001, 123, 8239-8247. (b) A. P. Côté, A. I. Benin, N. W. Ockwig, M. O’Keeffe, A. J. Matzger, O. M. Yaghi, Science, 2005, 310, 1166-1170. (c) H. M. El-Kaderi, J. R. Hunt, J. L. MendozaCortés, A. P. Côté, R. E. Taylor, M. O’Keeffe, O. M. Yaghi, Science, 2007, 316, 268-272. (d) F. J. UribeRomo, J. R. Hunt, H. Furukawa, C. Klöck, M. O’Keeffe, O. M. Yaghi, J. Am. Chem. Soc. 2009, 131, 4570-4571. (e) D. Beaudoin, T. Maris, J. D. Wuest, Nat. Chem. 2013, 5, 830-834. (f) Q. Fang, J. Wang, 
S. Gu, R. B. Kaspar, Z. Zhuang, J. Zheng, H. Guo, S. Qiu, Y. Yan, J. Am. Chem. Soc. 2015, 137, 83528355.

10 (a) E. Stöckel, X. Wu, A. Trewin, C. D. Wood, R. Clowes, N. L. Campbell, J. T. A. Jones, Y. Z. Khimyak, D. J. Adams, A. I. Cooper, Chem. Commun. 2009, 212-214. (b) O. K. Farha, A. M. Spokoyny, B. G. Hauser, Y.-S. Bae, S. E. Brown, R. Q. Snurr, C. A. Mirkin, J. T. Hupp, Chem. Mater. 2009, 21, 3033-3035. (c) T. Ben, H. Ben, S. Ma, D. Cao, J. Lan, X. Jing, W. Wang, J. Xu, F. Deng, J. M. Simmons, S. Qiu, G. Zhu, Angew. Chem. Int. Ed. 2009, 48, 9457-9460. (d) J. R. Holst, E. Stöckel, D. J. Adams, A. I. Cooper, Macromolecules 2010, 43, 8531-8538. (e) Y. Zhu, H. Yang, Y. Ji, W. Zhang, Chem. Mater. 2013, 25, 3718-3723.

11 (a) J. I. Urzúa, M. A. Regueira, M. Lazzari, M. Torneiro, Polym. Chem. 2016, 7, 5641-5645. (b) J. I. Urzúa, M. Torneiro, J. Org. Chem. 2017, 82, 13231-13238.

12 U. Ziener, A. Godt, J. Org. Chem. 1997, 62, 6137-6143.

13 (a) Q. Li, A. V. Rukavishnikov, P. A. Petukhov, T. O. Zaikova, , J. F. W. Keana Org. Lett. 2002, 4, 3631-3634. (b) D. Su, F. M. Menger, Tetrahedron Lett. 1997, 38, 1485-1488.

14 J. G. Rodríguez, J. L. Tejedor, T. La Parra, C. Díaz, Tetrahedron 2006, 62, 3355-3361.

15 C. Glaser, Ber. Dtsch. Chem. Ges. 1869, 2, 422-424.

16 K. Balaraman, V. Kesavan, Synthesis 2010, 20, 3461-3466.

17 Q.Zheng, R. Hua, Y. Wan, Appl. Organomet. Chem. 2010, 24, 314-316.

18 For general procedures see reference 11a. 


\title{
Probing the versatility of shape-persistent
}

\section{tetraphenylmethane dendrimers by modification of the}

\section{skeleton.}

\author{
Julio I. Urzúa, ${ }^{\mathrm{a}, \uparrow}$ Sandra Campana, ${ }^{\mathrm{a}}$ Massimo Lazzari ${ }^{\mathrm{b}}$ and Mercedes Torneiro ${ }^{\mathrm{a} *}$
}

a'Departamento de Química Orgánica, Facultade de Química, Universidade de Santiago de Compostela, 15782, Santiago de Compostela, Spain.

${ }^{b}$ Centro Singular de Investigación en Química Biolóxica e Materiais Moleculares (CIQUS) and

Departamento de Química Física, Universidade de Santiago de Compostela, 15782, Santiago de Compostela, Spain.

*E-mail: mercedes.torneiro@usc.es

$\dagger$ Present address: Comisión Chilena de Energía Nuclear, calle Nueva Bilbao 12501, Comuna de los Condes, 7600713, Santiago de Chile, Chile.

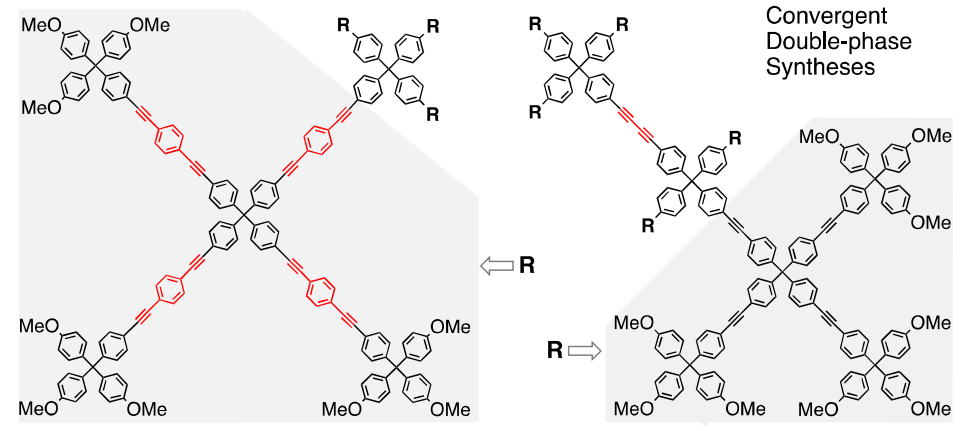


ABSTRACT. Tetraphenylmethane has emerged as a recurrent building block for advanced porous materials such as COFs, PAFs and hypercrosslinked polymers. Guided by a similar design principle, we have previously synthesized shape-persistent dendrimers with tetraphenylmethane nodes and ethynylene linkers. Here we report the generality of our approach by describing new dendritic architectures built from tetraphenylmethane. First, we prepared expanded dendrimers where the tetrahedral units are bonded through larger rigid rod spacers. Among the different synthetic strategies tested, the convergent route, with alternating steps of Pd-catalyzed Sonogashira coupling and alkyne activation by removal of TMS masking groups, efficiently afforded the first- and second-generation dendrimers. A second type of compounds having a linear diyne at the core is also described. The dendrimers of generations 1-2 were also synthesized by a convergent approach, with the diyne being assembled in the last step of the synthesis by a Glaser oxidative homocoupling of the corresponding dendrons bearing a terminal alkyne at the focal point. A third-generation dendrimer was also successfully prepared by a double-phase strategy.

\section{Introduction}

The tetrahedral tetravalent structure of $\mathrm{sp}^{3}$ carbon is responsible, in conjunction with the diversity of functional groups and the stability of the $\mathrm{C}-\mathrm{C}$ bond, of the structural and functional variety of organic compounds. Tetraphenylmethane can be considered as an expanded $\mathrm{sp}^{3}$ carbon that reproduces the same tetrahedral geometry in a larger structure when radially substituted, i.e. at the para positions of the aromatic rings. In fact, tetraphenylmethane is a rigid three-dimensional building block widely used to assemble the structural framework of artificial systems of considerable size. To name just a few, it has been employed to build tetrahedral structures, ${ }^{1}$ in self-assembled and supramolecular systems, ${ }^{2}$ as 
building block for light-emitting and antennae materials, ${ }^{3}$ in electronically active materials, ${ }^{4}$ in liquid crystals, $^{5}$ for rotaxane stoppers ${ }^{6}$ and as core of shape-persistent dendrimers. ${ }^{7}$ A field of recent development where tetraphenylmethane has been extensively employed is that of organic porous materials. In particular, it has been used as building block for molecular cages, ${ }^{8}$ reticular materials (MOFs and COFs) ${ }^{9}$ and amorphous porous frameworks (microporous polymers, PAFs and related materials). ${ }^{10}$ In this last case, the tetraphenylmethane units are hypercrosslinked either directly or through linear spacers to create diamond-related amorphous frameworks with internal voids or pores resulting from the radial stiffness and inefficient packing of the building blocks.

We have recently reported the synthesis and fluorescent properties of shape-persistent dendrimers with tetraphenylmethane nodes and ethynylene linkers of generations 1 and 2 (e.g. G1a, Fig. 1). ${ }^{11}$ The interest of these macromolecules lies in their privileged architectures, with a protein-like globular structure from the second generation. They have a hydrophobic interior with well-defined cavities and the polar terminal groups are effectively located at the periphery with reduced possibilities of backfolding. Being the soluble and structurally-regular analogs of microporous polymers, dendrimers of this type can be potentially used for separation technologies, for gas storage, in catalysis, sensing, and to build multichromophoric luminescent materials or nanomachines.

In this paper, we investigate the scope of the convergent, divergent and double-phase synthetic methodologies to access new members of this family of macromolecules. In addition, we show the versatility of this modular design by preparing dendrimers with modifications at their skeleton, either at the branches or the core. In the first case, our goal is the synthesis of molecules with an expanded structure, which can be accomplished by introducing larger rigid rod linkers between the tretraphenylmethane nodes. As an example, we selected for this work dendrimers G1b and G2b (Fig. 1), 
which have ethynylene-phenylene-ethynylene spacers. As a consequence, they have bigger size and molecular weight than the parent compounds (G1a and the analog second-generation dendrimer), while maintaining the shape-persistent structure. Three-dimensional models show that G1b has a star-like shape, whereas G2b has a globular structure with well-defined inner cavities of bigger volume then the parent compound. On the other side, we also describe in this work dendrimers G1c, G2c and G3c (Fig. 1), with a diyne core. The overall structure is more similar to the related tetraphenylmethane-cored prototypes (e.g. G1a), but with an ovoid instead of spherical globular shape resulting from the linear symmetry of the core. 

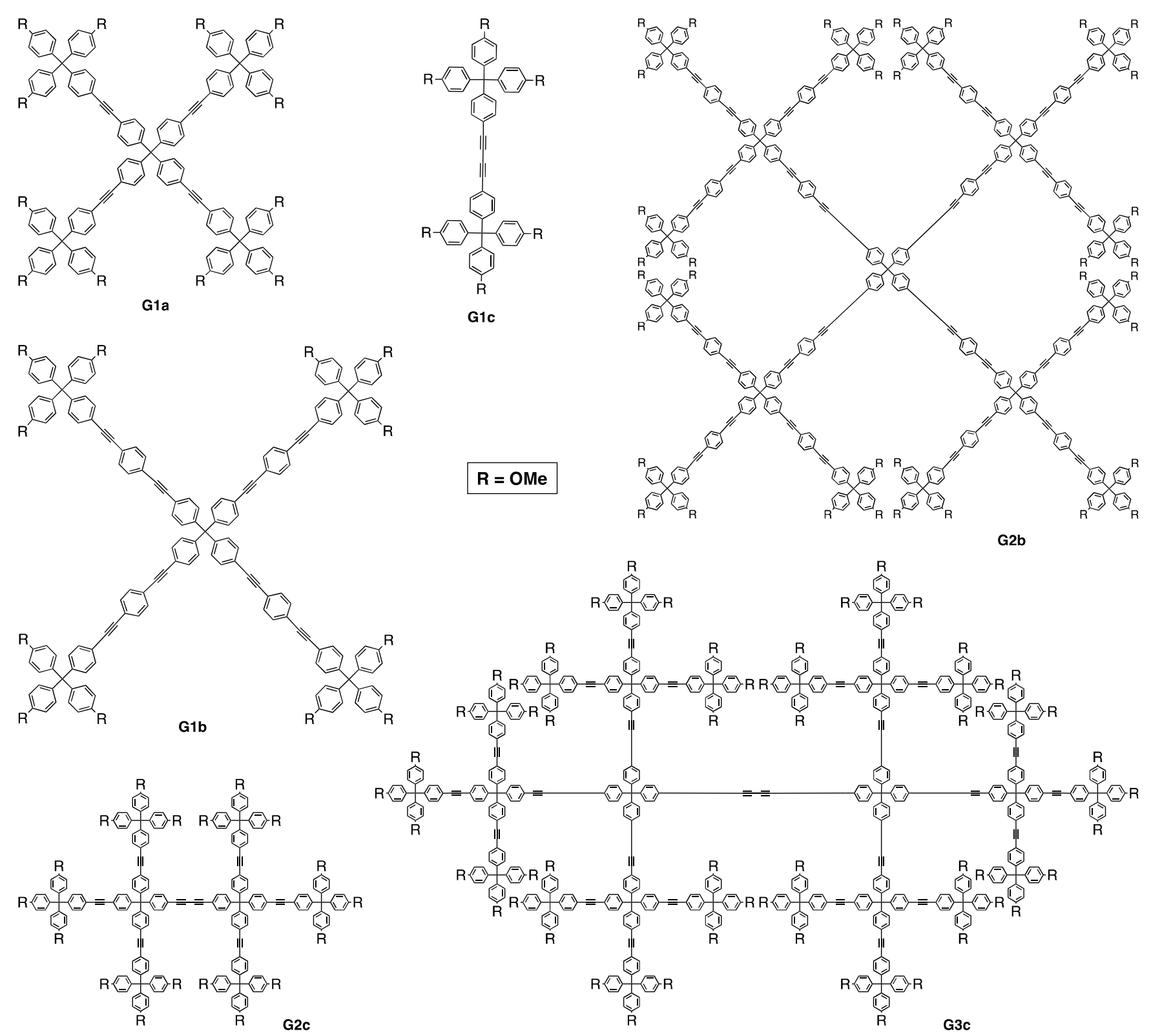

Figure 1. Structure of dendrimers of the $\mathbf{a}(\mathbf{G 1 a}), \mathbf{b}(\mathbf{G 1 b}, \mathbf{G 2 b})$ and ceries $(\mathbf{G 1 c}, \mathbf{G 2 c}, \mathbf{G 3 c})$.

\section{Results and Discussion}

We started our work with the synthesis of the b series macromolecules. We envisaged that the expanded dendrimers could be easily accessed by a convergent route based on Sonogashira Pd-catalyzed couplings, previously demonstrated in our group for the prototype tetraphenylmethane dendrimers with ethynylene linkers. ${ }^{11 a}$ The first generation dendron or terminal unit (4, Scheme 1) was prepared by coupling of the alkyne $\mathbf{1}^{11}$ with the aryl bromide $2^{12}$ using $\mathrm{Pd}\left(\mathrm{PPh}_{3}\right)_{4}$ as catalyst in the presence of $\mathrm{PPh}_{3}$, 
$\mathrm{CuI}$ and $4 \AA$ molecular sieve in piperidine at $100{ }^{\circ} \mathrm{C}$, followed by removal of the TMS group at the focal point by treatment with sodium hydroxide in methanol. A new Sonogashira coupling of the dendron 4 with the core tetraiodide $\mathbf{5},{ }^{11,13}$ using the standard preceding conditions, afforded the first-generation dendrimer G1b in excellent $83 \%$ yield for the three steps.

\section{Scheme 1. Convergent route to dendrimers G1b and G2b.}

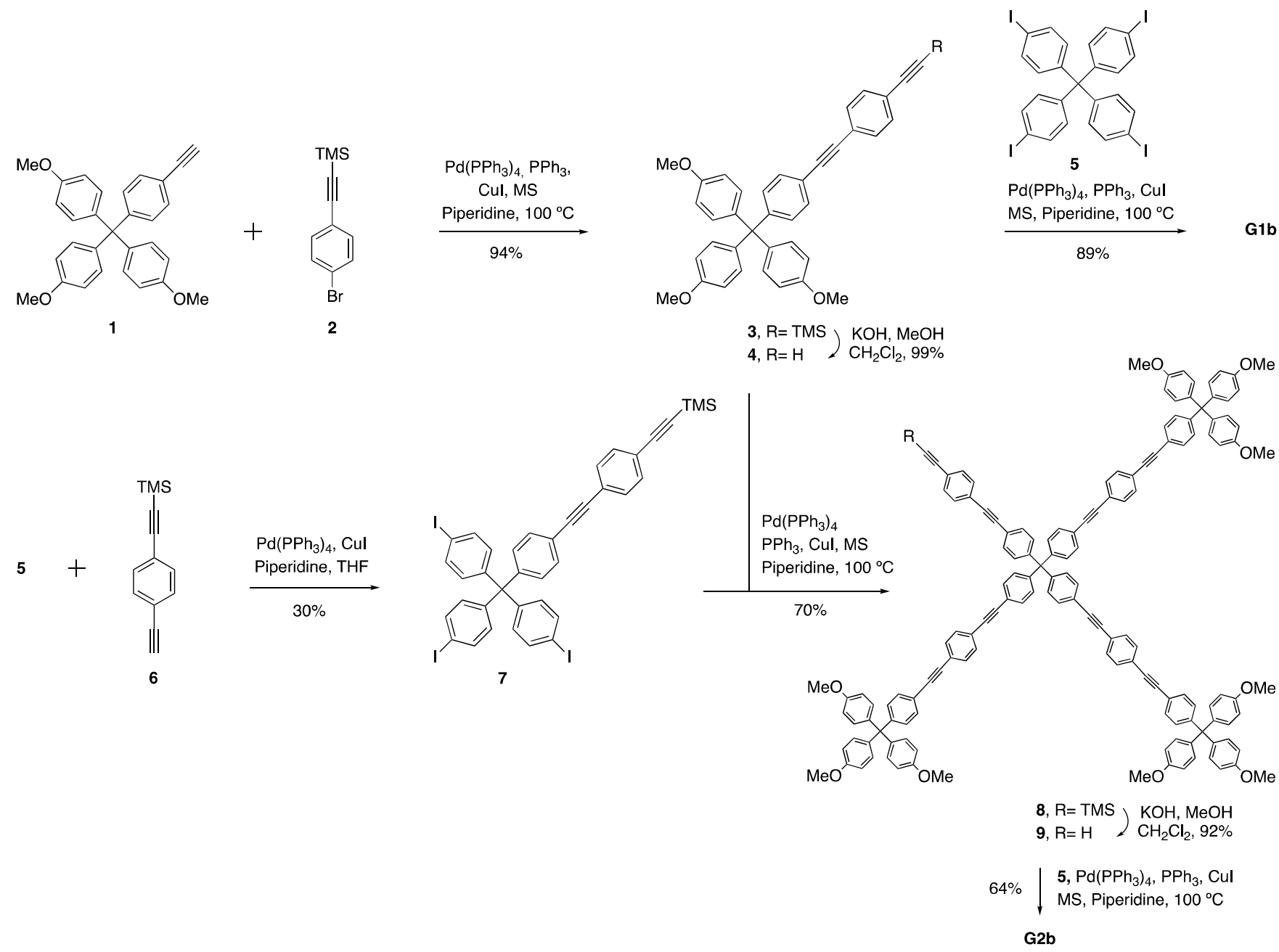

To address the synthesis of the second-generation dendrimer, we first prepared the branching unit 7 by coupling of the acetylene $6^{14}$ and the tetraiodide 5 using $\mathrm{Pd}\left(\mathrm{PPh}_{3}\right)_{4}, \mathrm{PPh}_{3}, \mathrm{CuI}$ and piperidine in $\mathrm{THF}$ at rt. The low yield in this reaction $(30 \%)$ is caused by the statistical reaction of the spacer with the aryl iodides of the tetravalent core. To avoid the formation of undesirable amounts of by-products resulting 
from coupling of multiple acetylenes to the core, an excess of the core building block $\mathbf{5}$ was used, which can be easily separated as the insoluble material in the reaction crude. The Pd-catalyzed coupling of the branching and the terminal units, $\mathbf{7}$ and $\mathbf{4}$, respectively, under standard conditions gave, in good yield, the protected second generation dendron, 8. Desilylation with $\mathrm{NaOH}$ and Sonogashira coupling with the core unit $\mathbf{5}$ afforded in good yield the second-generation dendrimer G2b. G1b and G2b were fully characterized by MALDI-TOF mass spectrometry and ${ }^{1} \mathrm{H} /{ }^{13} \mathrm{C}$ NMR spectroscopy and they showed a clean SEC trace with a single peak with retention times according to their molecular weights. A common property of this series of expanded dendrimers is their low solubility, that is useful for their isolation, as they precipitate in the reaction media as white solids. They are very stable, remaining unchanged after several days exposed to air on the bench. In addition, they show a characteristic luminescence that can be observed as a purple glow by illuminating a dilute solution under an UV lamp or as a bright blue spot upon UV irradiation of a sample on a TLC plate. They show a good stability,

\section{Scheme 2. Divergent and double-phase strategies for the $b$ series of dendrimers.}

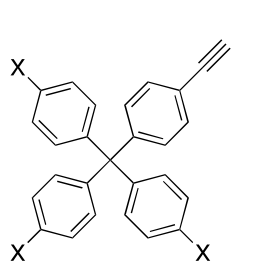

10

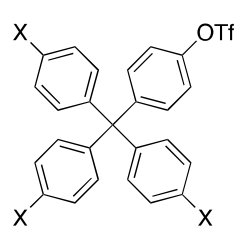

11
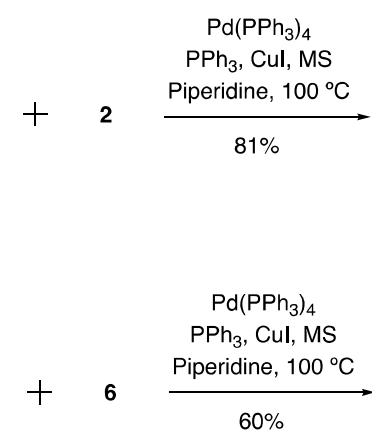

$\mathrm{X}=\{-\mathrm{N}$

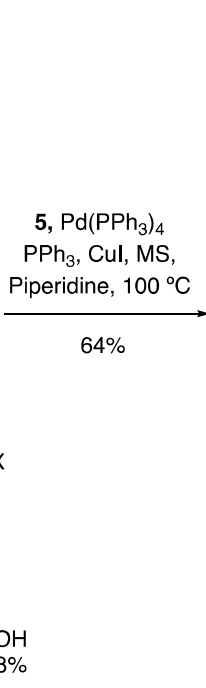

12, $\mathrm{R}=\mathrm{TMS} \quad \mathrm{KOH}, \mathrm{MeOH}$ 13, $\mathrm{R}=\mathrm{H} \quad \mathrm{CH}_{2} \mathrm{Cl}_{2}, 98 \%$

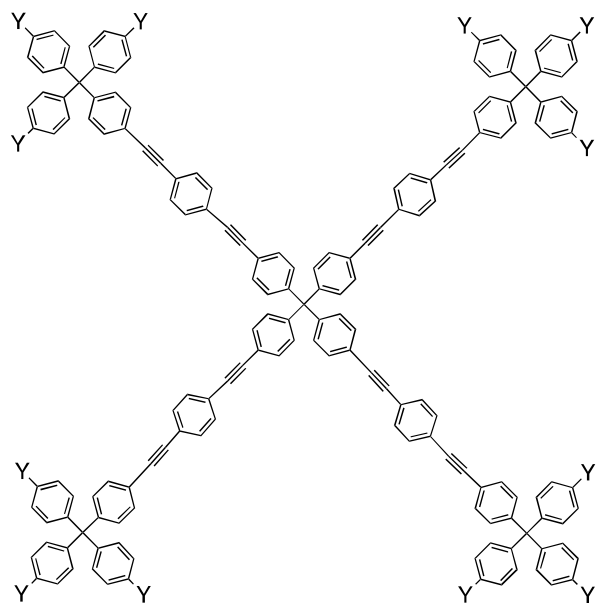

G1b- $\left.N_{3}, Y=X\right) \quad M e l, 120^{\circ} \mathrm{C}$ G1b-I, $Y=1 \quad 95 \%$

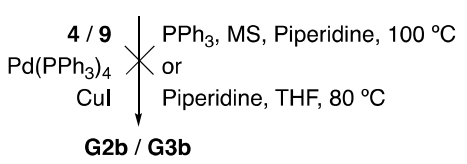


At this point, we decided to apply our recently developed triazene-based divergent route ${ }^{11 \mathrm{~b}}$ to this series of dendrimers for comparative purposes. Therefore, we explored the divergent approach to G2b using as starting tetraphenylmethane building blocks the tris(triazene) derivatives $\mathbf{1 0}^{11 \mathrm{~b}}$ and $\mathbf{1 1}^{11 \mathrm{~b}}$ that were coupled to either spacer $\mathbf{2}$ or $\mathbf{6}$, respectively (Scheme 2). In both cases the desired protected expanded dendron 12 was obtained with good to moderate yields. Subsequent desilylation and coupling to tetraiodide $\mathbf{5}$ afforded the triazene-terminated first generation dendrimer $\mathbf{G 1 b}-\mathbf{N}_{\mathbf{3}}$ in $63 \%$ yield for the two steps, which was transformed in the iodo-terminated dendrimer G1b-I by treatment with MeI at 120 ${ }^{\circ} \mathrm{C}$ (95\% yield). Unfortunately, our efforts to grow this dendrimer by coupling with dendron 4 were unsuccessful, yielding complex mixtures, in which compound $\mathbf{G} 2 \mathbf{b}$ could not be detected. The reaction of G1b-I with dendron 9 was also tested in an attempt to prepare the corresponding third-generation dendrimer (G3b, structure not shown) by a double-stage method, but the desired dendrimer could not be detected in the resulting complex reaction mixtures.

Next, we turned our attention to the synthesis and characterization of the diyne-cored dendrimers of the $\mathbf{c}$ series. In addition to their interesting structure, these compounds should be easily accessible by Glaser oxidative homocoupling ${ }^{15}$ of the corresponding dendrons bearing a terminal alkyne at the focal point. Of the many conditions reported in the literature for this reaction, we selected those that were favorable to the dissolution of the substrates. We started by testing the reaction of $\mathbf{1}$ with different copper salts as catalyst in $\mathrm{CH}_{2} \mathrm{Cl}_{2},{ }^{16}$ but neither $\mathrm{CuI}$ or $\mathrm{CuCl}$ in combination with diethylamine, piperidine or pyrrolidine as bases and in the presence of oxygen gave the desired product G1c, and the starting material was recovered unaltered. Similar negative results were obtained when the reaction was carried out with Hua's conditions ${ }^{17}-\mathrm{CuCl}$ and piperidine in toluene. 
Scheme 3. Convergent route to dendrimers G1c and G2c.

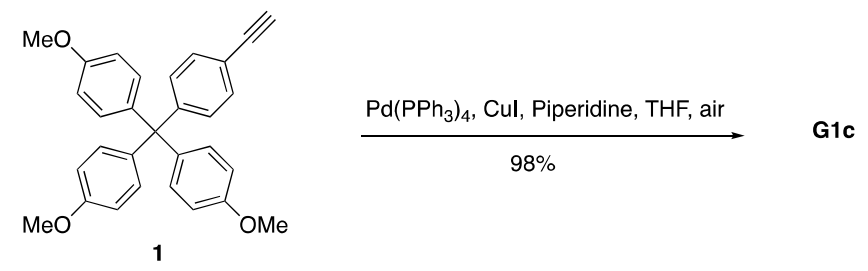

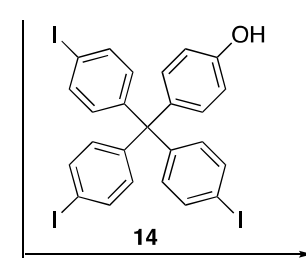

$\mathrm{Pd}\left(\mathrm{PPh}_{3}\right)_{4}, \mathrm{PPh}_{3}, \mathrm{Cul}, \mathrm{MS}$ Piperidine, $100^{\circ} \mathrm{C}, 73 \%$

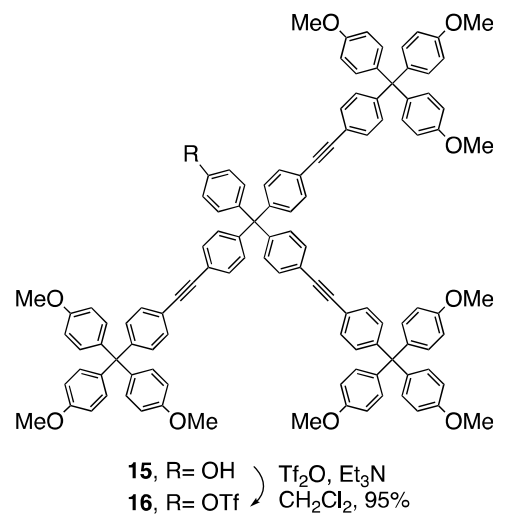

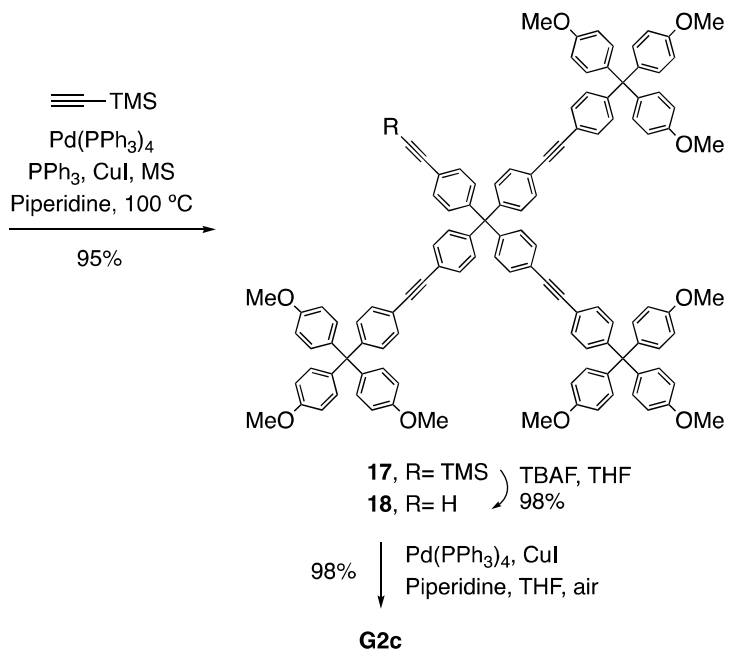

Our observation during the synthesis of the tetraphenylmethane-cored dendrimers (e.g. G1a) that small amounts of the diyne-cored dendrimers were sometimes obtained as by-products, led us to essay the Sonogashira conditions under air at rt. So, the reaction of 1 with $\mathrm{Pd}\left(\mathrm{PPh}_{3}\right)_{4}(0.1$ eq. $)$, CuI (0.1 eq.) and piperidine (13 eq.) in THF in the presence of air afforded the desired dendrimer G1c in low yield, which was improved to $98 \%$ yield by increasing the amount of $\mathrm{CuI}$ to 2 eq. (Scheme 3). To undertake the synthesis of the dendrimer G2c, we first developed a new synthetic route to the protected secondgeneration dendron 17. Instead of coupling the spacer to the tetraiodide 5 with $\sim 30 \%$ yield, as in the previously reported method, ${ }^{11 a}$ and in the strategy followed to prepare $\mathbf{8}$ (Scheme 1), we reacted the terminal unit 1 to the known triiodide $14^{6}$ to obtain the phenol 15 in $73 \%$ yield (Scheme 3). Treatment with triflic anhydride gave the intermediate triflate 16, which was coupled with the TMSA spacer to yield the desired protected dendron 17 (90\% for the two steps). Desilylation to give the dendron $\mathbf{1 8}^{11 a}$ 
followed by Glaser homocoupling in the above optimized conditions afforded the final secondgeneration dendrimer G2c in excellent $96 \%$ yield (two steps).

Once demonstrated the feasibility of the convergent method to access the c series of dendrimers, we considered worth to attempt the double-stage strategy for the synthesis of a dendrimer of third generation. The Glaser homocoupling of the tris(triazene) $\mathbf{1 0}$ gave the triazene terminated dendrimer G1c-N $\mathbf{N}_{\mathbf{3}}$ in excellent yield (Scheme 4). Treatment with methyl iodide afforded the hexaiodide G1c-I that worked as a supercore for dendrimer groth by Sonogashira reaction with dendron 18. In this case, the double-stage coupling proceeded smoothly to give the dendrimer G3c in $66 \%$ yield for the last two steps.

Scheme 4. Double-phase synthesis of G3c.
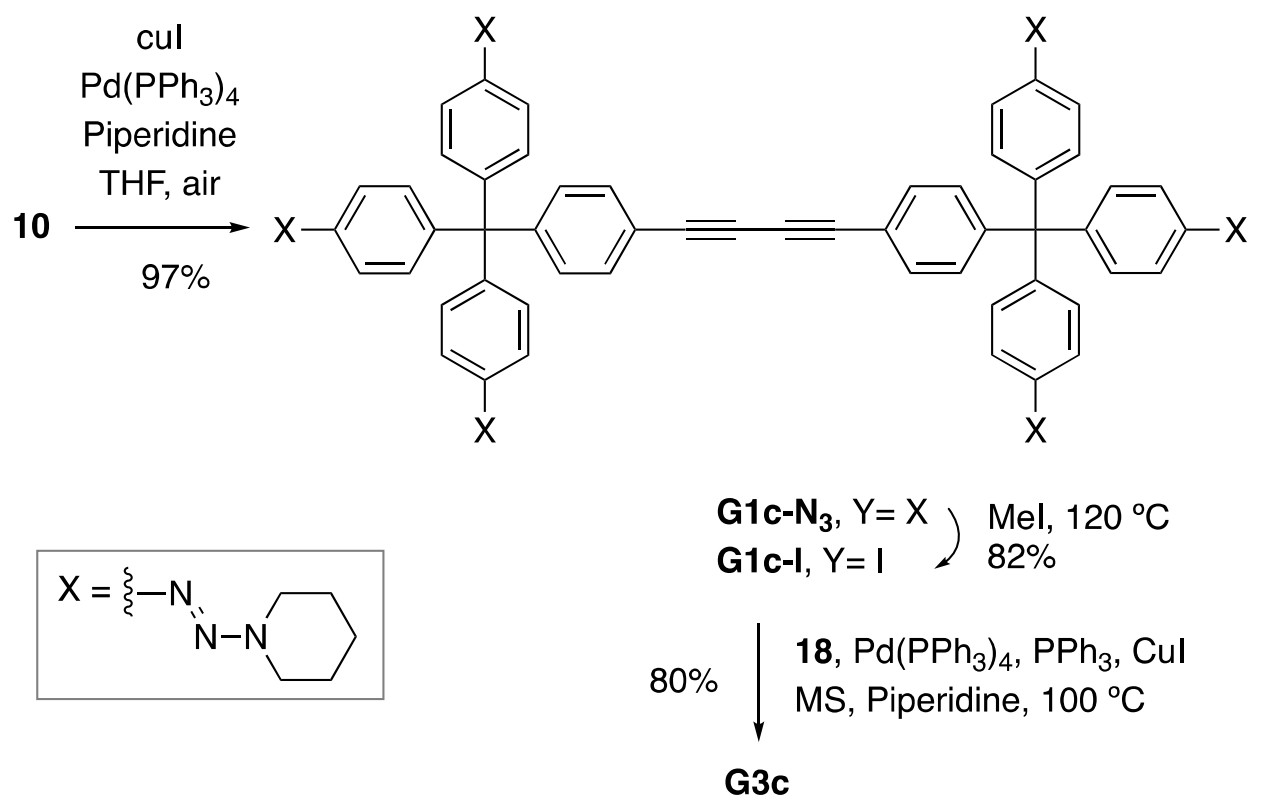

Dendrimers G1c, G2c and G3c were conveniently characterized $\left({ }^{1} \mathrm{H}\right.$ and ${ }^{13} \mathrm{C}$ NMR, MALDI-TOF MS $)$ and showed a clean SEC trace with retention times according to their sizes. They are stable white solids 
compounds with good solubility in common solvents (THF, $\mathrm{CH}_{2} \mathrm{Cl}_{2}, \mathrm{CHCl}_{3}$ ). By visual inspection they do not show luminiscent properties as in the case of the previous series.

In conclusion, we have shown that tetraphenylmethane-based shape persistent dendrimers have very versatile modular structures that are accessible by different synthetic methodologies. In this work we have prepared two new series of compounds with unique geometrical characteristics, either with a expanded structure or with a diyne core. Both families were easily prepared by a convergent strategy. Whereas the convergent and double-phase routes were unsuccessful for the synthesis of $\mathbf{G 2 b}$ and $\mathbf{G 3 b}$, respectively, G3b was efficiently prepared by a double-stage methodology. The chemistry involved in those syntheses is basically an iterative process based on Pd-catalyzed Sonogashira couplings of terminal alkynes with aryl iodides and triflates, with alternating steps of activation of masked groups. In the case of the c series there is also a key step consisting of a Glaser homodimerization to assemble the core diyne. This is the first example of a double-stage strategy for rigid tetraphenylmethane dendrimers, and also we report the first dendrimer of third generation of this class. Further studies are being carried out in our laboratories to evaluate the properties (e.g. porosity, fluorescence) of these new macromolecular materials, which will be reported in future papers.

\section{Experimental Section ${ }^{18}$}

Protected dendron 3. A Schlenk tube equipped with a stirring bar was charged with $\mathbf{1}^{11}$ (200 mg, 0.46 mmol), $2^{12}$ (175 mg, $\left.0.69 \mathrm{mmol}\right), \mathrm{CuI}(4 \mathrm{mg}, 0.023 \mathrm{mmol}), \mathrm{Ph}_{3} \mathrm{P}(6 \mathrm{mg}, 0.023 \mathrm{mmol})$ and $\left(\mathrm{Ph}_{3} \mathrm{P}\right)_{4} \mathrm{Pd}(27$ $\mathrm{mg}, 0.023 \mathrm{mmol})$. After vacuum-drying, activated $4 \AA$ molecular sieves and piperidine $(15 \mathrm{~mL})$ were added under argon. The Schlenk tube was sealed with a teflon screw cap and the reaction mixture was stirred at $100{ }^{\circ} \mathrm{C}$ during $12 \mathrm{~h}$ and then it was allowed to reach rt. The mixture was concentrated and the residue was dissolved in $\mathrm{CH}_{2} \mathrm{Cl}_{2}(20 \mathrm{~mL})$ and washed with a saturated solution of $\mathrm{NH}_{4} \mathrm{Cl}(3 \times 15 \mathrm{~mL})$. 
The organic layer was dried, filtered and concentrated and the residue was purified by flash chromatography $\left(\mathrm{SiO}_{2}, 5 \%\right.$ EtOAc/hexanes) to give 3 as a white solid [267 $\mathrm{mg}, 0.44 \mathrm{mmol}, 94 \%$, $\mathrm{Rf}=$ 0.59 (20\% EtOAc/hexanes)]. ${ }^{1} \mathrm{H} \mathrm{NMR}\left(\mathrm{CDCl}_{3}, 250 \mathrm{MHz}\right): 7.43$ (4H, s, Ar), $7.41(2 \mathrm{H}, \mathrm{d}, J=8.3 \mathrm{~Hz}, \mathrm{Ar})$ $7.20(2 \mathrm{H}, \mathrm{d}, J=8.3 \mathrm{~Hz}, \mathrm{Ar}), 7.10(6 \mathrm{H}, \mathrm{d}, J=8.8 \mathrm{~Hz}, \mathrm{Ar}), 6.80(6 \mathrm{H}, \mathrm{d}, J=8.8 \mathrm{~Hz}$ Ar), $3.80(9 \mathrm{H}, \mathrm{s}, \mathrm{OMe})$, 0.27 (9H, s, TMS). ${ }^{13} \mathrm{C} \mathrm{NMR}\left(\mathrm{CDCl}_{3}, 63 \mathrm{MHz}\right): 157.1(\mathrm{C}), 147.8$ (C), 138.6 (C), $131.6(\mathrm{CH}), 131.5$ (CH), $130.9(\mathrm{CH}), 130.3(\mathrm{CH}), 123.0(\mathrm{C}), 122.4(\mathrm{C}), 119.9(\mathrm{C}), 112.4(\mathrm{CH}), 104.3(\mathrm{C}), 95.8(\mathrm{C}), 90.9$ (C), $88.6(\mathrm{C}), 62.5(\mathrm{C}), 54.8\left(\mathrm{CH}_{3}\right), 0.44$ (TMS). MS (EI, m/z, \%): $606.0\left(\mathrm{M}^{+}, 75\right), 557.9$ (16), 499.0 $\left(\mathrm{M}^{+}-\mathrm{C}_{7} \mathrm{H}_{7} \mathrm{O}, 77\right), 333.0\left(\mathrm{M}^{+}-\mathrm{C}_{19} \mathrm{H}_{17} \mathrm{Si}, 100\right)$. HRMS (EI) calcd. for $\mathrm{C}_{41} \mathrm{H}_{38} \mathrm{O}_{3} \mathrm{Si}\left(\mathrm{M}^{+}\right)$606.2590, found 606.2590.

Dendron 4. To a solution of $3(100 \mathrm{mg}, 0.17 \mathrm{mmol})$ in $\mathrm{CH}_{2} \mathrm{Cl}_{2}(10 \mathrm{~mL})$ and $\mathrm{MeOH}(4 \mathrm{~mL})$ was added $\mathrm{KOH}(93 \mathrm{mg}, 1.7 \mathrm{mmol})$ under argon and the mixture was stirred at $\mathrm{rt}$ during $1 \mathrm{~h}$. The mixture was concentrated and the residue was dissolved in EtOAc $(20 \mathrm{~mL})$. The resulting solution was washed with brine $(3 \times 30 \mathrm{~mL})$, dried and concentrated to give a residue that was dissolved in $\mathrm{CH}_{2} \mathrm{Cl}_{2}$. Precipitation with hexanes gave 4 as a white solid [87 mg, $0.16 \mathrm{mmol}, 99 \%, \mathrm{Rf}=0.48(20 \%$ EtOAc/hexanes $)] .{ }^{1} \mathrm{H}$ NMR (CDCl $3,250 \mathrm{MHz}): 7.47(4 \mathrm{H}, \mathrm{s}, \mathrm{Ar}), 7.42(2 \mathrm{H}, \mathrm{d}, J=8.2 \mathrm{~Hz}, \mathrm{Ar}), 7.21(2 \mathrm{H}, \mathrm{d}, J=8.2 \mathrm{~Hz}, \mathrm{Ar}), 7.10$ $\left(6 \mathrm{H}, \mathrm{d}, J=8.7 \mathrm{~Hz}, \mathrm{Ar}_{\mathrm{OMe}}\right), 6.80\left(6 \mathrm{H}, \mathrm{d}, J=8.7 \mathrm{~Hz}, \mathrm{Ar}_{\mathrm{OMe}}\right), 3.79(9 \mathrm{H}, \mathrm{s}, \mathrm{OMe}), 3.18(1 \mathrm{H}, \mathrm{s}, \mathrm{C} \equiv \mathrm{C}-\mathrm{H}) .{ }^{13} \mathrm{C}$ NMR (CDCl $\left.{ }_{3}, 63 \mathrm{MHz}\right): 157.1(\mathrm{C}), 147.9(\mathrm{C}), 138.6(\mathrm{C}), 131.6(\mathrm{CH}), 131.5(\mathrm{CH}), 131.0(\mathrm{CH}), 130.6$ (CH), $130.3(\mathrm{CH}), 123.4(\mathrm{C}), 121.3(\mathrm{C}), 119.8(\mathrm{C}), 112.3(\mathrm{CH}), 91.0(\mathrm{C}), 88.4(\mathrm{C}), 82.9(\mathrm{C}), 78.5(\mathrm{C})$, $62.5(\mathrm{C}), 54.8\left(\mathrm{CH}_{3}\right)$. MS (EI, m/z, \%): $534.0\left(\mathrm{M}^{+}, 78\right), 427.0\left(\mathrm{M}^{+}-\mathrm{C}_{7} \mathrm{H}_{7} \mathrm{O}, 85\right), 333.0\left(\mathrm{M}^{+}-\mathrm{C}_{16} \mathrm{H}_{9}, 100\right)$. HRMS (EI) calcd. for $\mathrm{C}_{38} \mathrm{H}_{30} \mathrm{O}_{3}\left(\mathrm{M}^{+}\right)$534.2195, found 534.2195.

Dendrimer G1b. A Schlenk tube equipped with a stirring bar was charged with freshly prepared 4 (176 mg, $0.33 \mathrm{mmol}), \mathbf{5}^{11,13}(60 \mathrm{mg}, 0.073 \mathrm{mmol}), \mathrm{CuI}(6 \mathrm{mg}, 0.029 \mathrm{mmol}), \mathrm{Ph}_{3} \mathrm{P}(8 \mathrm{mg}, 0.029 \mathrm{mmol})$ 
and $\left(\mathrm{Ph}_{3} \mathrm{P}\right)_{4} \mathrm{Pd}(34 \mathrm{mg}, 0.029 \mathrm{mmol})$. After vacuum-drying, activated $4 \AA$ molecular sieves and piperidine $(15 \mathrm{~mL})$ were added under argon. The Schlenk tube was sealed with a teflon screw cap and the reaction mixture was stirred at $100{ }^{\circ} \mathrm{C}$ during $12 \mathrm{~h}$. Then it was allowed to reach rt to give a white precipitate which was filtered and dissolved in hot $\mathrm{CH}_{2} \mathrm{Cl}_{2}(30 \mathrm{~mL})$. The resulting solution was allowed to slowly reach rt and a white precipitate was formed, which was filtered to give dendrimer G1b as a white solid [160 mg, $0.065 \mathrm{mmol}, 89 \%$ ]. ${ }^{1} \mathrm{H} \mathrm{NMR}\left(\mathrm{CDCl}_{3}, 250 \mathrm{MHz}\right): 7.46$ (32H, m, Ar), 7.19 (16H, m, Ar), $7.08\left(24 \mathrm{H}, \mathrm{d}, J=8.7 \mathrm{~Hz}, \mathrm{Ar}_{\mathrm{OMe}}\right), 6.78\left(24 \mathrm{H}, \mathrm{d}, J=8.8 \mathrm{~Hz} \mathrm{Ar}_{\mathrm{OMe}}\right), 3.79(36 \mathrm{H}, \mathrm{s}, \mathrm{OMe}) .{ }^{13} \mathrm{C} \mathrm{NMR}$ $\left(\mathrm{CDCl}_{3}, 63 \mathrm{MHz}\right)$ : $157.4(\mathrm{C}), 148.1(\mathrm{C}), 145.8(\mathrm{C}), 138.8(\mathrm{C}), 131.8(\mathrm{CH}), 131.4(\mathrm{CH}), 130.8(\mathrm{CH})$, $130.5(\mathrm{CH}), 123.1(\mathrm{C}), 122.7(\mathrm{C}), 121.0(\mathrm{C}), 120.1(\mathrm{C}), 112.6(\mathrm{CH}), 91.1(\mathrm{C}), 90.6(\mathrm{C}), 89.5(\mathrm{C}), 88.9$ (C), $64.4(\mathrm{C}), 62.7(\mathrm{C}), 55.0\left(\mathrm{CH}_{3}\right)$. MS (MALDI-TOF, m/z): $2559\left(\mathrm{MAg}^{+}\right), 2450\left(\mathrm{M}^{+}\right), 2343\left(\mathrm{M}^{+}-\right.$ $\left.\mathrm{C}_{7} \mathrm{H}_{7} \mathrm{O}\right), 1840\left(\mathrm{M}^{+}-\mathrm{C}_{44} \mathrm{H}_{33} \mathrm{O}_{3}\right)$.

Trimehyl((4-((4-(tris(4-iodophenyl)methyl)phenyl)ethinyl)phenyl)ethinyl)silane (7). A mixture of $\mathbf{6}^{14}$ (77 mg, $\left.0.39 \mathrm{mmol}\right), \mathbf{5}^{11,13}(400 \mathrm{mg}, 0.49 \mathrm{mmol}), \mathrm{CuI}(9 \mathrm{mg}, 0.049 \mathrm{mmol})$ and $\left(\mathrm{Ph}_{3} \mathrm{P}\right)_{4} \mathrm{Pd}(56 \mathrm{mg}$, $0.049 \mathrm{mmol})$ was dissolved in THF $(50 \mathrm{~mL})$ by refluxing under argon. Then, the solution was allowed to reach $\mathrm{rt}$ and piperidine $(0.5 \mathrm{~mL}, 4.85 \mathrm{mmol})$ was added. After stirring for $12 \mathrm{~h}$, the mixture was concentrated, the residue was dissolved in $\mathrm{CH}_{2} \mathrm{Cl}_{2}(20 \mathrm{~mL})$ and the resulting solution was washed with a saturated solution of $\mathrm{NH}_{4} \mathrm{Cl}(3 \times 10 \mathrm{~mL})$. The organic phase was dried and concentrated and the residue was purified by flash chromatography ( $\mathrm{SiO}_{2}$, hexanes) to give 7 as a white solid [139 $\mathrm{mg}, 0.155 \mathrm{mmol}$, 30\%, Rf=0.33 (5\% $\mathrm{CH}_{2} \mathrm{Cl}_{2} /$ hexanes $\left.)\right] .{ }^{1} \mathrm{H} \mathrm{NMR}\left(\mathrm{CDCl}_{3}, 250 \mathrm{MHz}\right): 7.59\left(6 \mathrm{H}, \mathrm{d}, J=8.0 \mathrm{~Hz}, \mathrm{Ar}_{\mathrm{I}}\right), 7.42$ $(6 \mathrm{H}, \mathrm{m}, \mathrm{Ar}), 7.14(2 \mathrm{H}, \mathrm{d}, J=8.1 \mathrm{~Hz}, \mathrm{Ar}), 6.91\left(6 \mathrm{H}, \mathrm{d}, J=8.0 \mathrm{~Hz} \mathrm{Ar}_{\mathrm{I}}\right), 0.27(9 \mathrm{H}, \mathrm{s}, \mathrm{TMS}) .{ }^{13} \mathrm{C}$ NMR $\left(\mathrm{CDCl}_{3}, 63 \mathrm{MHz}\right)$ : $145.7(\mathrm{C}), 145.3(\mathrm{C}), 137.2(\mathrm{CH}), 132.8(\mathrm{CH}), 132.0(\mathrm{CH}), 131.5(\mathrm{CH}), 131.3(\mathrm{CH})$, $130.8(\mathrm{CH}), 123.2(\mathrm{C}), 123.1(\mathrm{C}), 121.4(\mathrm{C}), 104.7(\mathrm{C}), 96.5(\mathrm{C}), 92.6(\mathrm{C}), 90.8(\mathrm{C}), 89.8(\mathrm{C}), 64.4(\mathrm{C})$, 
0.08 (TMS). MS (EI, m/z, \%): $893.2\left(\mathrm{M}^{+}, 100\right), 690.4\left(\mathrm{M}^{+}-\mathrm{C}_{6} \mathrm{H}_{5} \mathrm{I}, 67\right)$. HRMS (EI) calcd. For $\mathrm{C}_{41} \mathrm{H}_{38} \mathrm{O}_{3} \mathrm{Si}$

${ }^{+}\left(\mathrm{M}^{+}\right)$893.9173, found 893.9178.

Protected dendron 8. A Schlenk tube equipped with a stirring bar was charged with 4 (330 mg, 0.62 mmol), 7 (158 mg, $0.18 \mathrm{mmol}), \mathrm{CuI}(5 \mathrm{mg}, 0.026 \mathrm{mmol}), \mathrm{Ph}_{3} \mathrm{P}(7 \mathrm{mg}, 0.026 \mathrm{mmol})$ and $\left(\mathrm{Ph}_{3} \mathrm{P}\right)_{4} \mathrm{Pd}(61$ $\mathrm{mg}, 0.053 \mathrm{mmol})$. After vacuum-drying, activated $4 \AA$ molecular sieves and piperidine $(20 \mathrm{~mL})$ were added under argon. The Schlenk tube was sealed with a teflon screw cap and the reaction mixture was stirred at $100{ }^{\circ} \mathrm{C}$ during $12 \mathrm{~h}$ and then it was allowed to reach $\mathrm{rt}$. The mixture was concentrated and the residue was dissolved in $\mathrm{CH}_{2} \mathrm{Cl}_{2}(20 \mathrm{~mL})$ and washed with a saturated solution of $\mathrm{NH}_{4} \mathrm{Cl}(3 \times 15 \mathrm{~mL})$. The organic layer was dried, filtered and concentrated and the residue was purified by flash chromatography $\left(\mathrm{SiO}_{2}, 40 \% \mathrm{CH}_{2} \mathrm{Cl}_{2} /\right.$ hexanes $)$ to give $\mathbf{8}$ as a white solid [260 $\mathrm{mg}, 0.12 \mathrm{mmol}, 70 \%, \mathrm{Rf}=$ $0.65\left(70 \%\right.$ toluene/ $\left.\left.\mathrm{CH}_{2} \mathrm{Cl}_{2}\right)\right] .{ }^{1} \mathrm{H} \mathrm{NMR}\left(\mathrm{CDCl}_{3}, 250 \mathrm{MHz}\right):$ 7.52-7.35 (30H, m, Ar), 7.21 (14H, m, Ar), $7.10\left(18 \mathrm{H}, \mathrm{d}, J=8.8 \mathrm{~Hz}, \mathrm{Ar}_{\mathrm{OMe}}\right), 6.80\left(18 \mathrm{H}, \mathrm{d}, J=8.8 \mathrm{~Hz} \mathrm{Ar}_{\mathrm{OMe}}\right), 3.80$ (27H, s, OMe), 0.27 (9H, s, TMS). ${ }^{13} \mathrm{C} \mathrm{NMR}\left(\mathrm{CDCl}_{3}, 63 \mathrm{MHz}\right): 157.4(\mathrm{C}), 148.1(\mathrm{C}), 145.9(\mathrm{C}), 138.9(\mathrm{C}), 131.8(\mathrm{CH}), 131.4(\mathrm{CH}), 131.0$ (CH), $130.9(\mathrm{CH}), 130.8(\mathrm{CH}), 130.5(\mathrm{CH}), 123.1(\mathrm{C}), 122.8(\mathrm{C}), 122.7(\mathrm{C}), 121.1(\mathrm{C}), 120.2(\mathrm{C}), 112.6$ (CH), $104.5(\mathrm{C}), 96.2(\mathrm{C}), 91.2(\mathrm{C}), 90.7(\mathrm{C}), 89.5(\mathrm{C}), 88.9(\mathrm{C}), 64.8(\mathrm{C}), 62.8(\mathrm{C}), 55.0\left(\mathrm{CH}_{3}\right), 0.21$ (TMS). MS (MALDI-TOF, m/z): $2113\left(\mathrm{M}^{+}\right), 2007\left(\mathrm{M}^{+}-\mathrm{C}_{7} \mathrm{H}_{7} \mathrm{O}\right), 1841\left(\mathrm{M}^{+}-\mathrm{C}_{19} \mathrm{H}_{17} \mathrm{Si}\right), 1504\left(\mathrm{M}^{+}-\right.$ $\left.\mathrm{C}_{44} \mathrm{H}_{33} \mathrm{O}_{3}\right)$.

Dendron 9. To a solution of $8(50 \mathrm{mg}, 0.024 \mathrm{mmol})$ in $\mathrm{CH}_{2} \mathrm{Cl}_{2}(5 \mathrm{~mL})$ and $\mathrm{MeOH}(2 \mathrm{~mL})$ was added $\mathrm{KOH}$ (14 mg, $0.24 \mathrm{mmol})$ under argon and the mixture was stirred at $\mathrm{rt}$ during $1 \mathrm{~h}$. The mixture was concentrated and the residue was dissolved in $\mathrm{CH}_{2} \mathrm{Cl}_{2}(20 \mathrm{~mL})$. The resulting solution was washed with brine $(3 \times 30 \mathrm{~mL})$, dried and concentrated to give a residue that was dissolved in $\mathrm{CH}_{2} \mathrm{Cl}_{2}$. Precipitation with hexanes gave 9 as a white solid [45 mg, $0.022 \mathrm{mmol}, 92 \%, \mathrm{Rf}=0.6\left(30 \%\right.$ toluene/ $\left.\left.\mathrm{CH}_{2} \mathrm{Cl}_{2}\right)\right]$. This 
dendron was immediately used in next reaction. ${ }^{1} \mathrm{H} \mathrm{NMR}\left(\mathrm{CDCl}_{3}, 250 \mathrm{MHz}\right)$ : 7.55-7.36 (30H, m, Ar), $7.21(14 \mathrm{H}, \mathrm{m}, \mathrm{Ar}), 7.09\left(18 \mathrm{H}, \mathrm{d}, J=8.7 \mathrm{~Hz}, \mathrm{Ar}_{\mathrm{OMe}}\right), 6.80\left(18 \mathrm{H}, \mathrm{d}, J=8.7 \mathrm{~Hz}, \mathrm{Ar}_{\mathrm{OMe}}\right), 3.80(27 \mathrm{H}, \mathrm{s}, \mathrm{OMe})$, $3.18(1 \mathrm{H}, \mathrm{s}, \mathrm{C} \equiv \mathrm{C}-\mathrm{H}) .{ }^{13} \mathrm{C} \mathrm{NMR}\left(\mathrm{CDCl}_{3}, 63 \mathrm{MHz}\right): 157.4(\mathrm{C}), 148.1(\mathrm{C}), 145.9(\mathrm{C}), 138.9$ (C), 131.9 $(\mathrm{CH}), 131.8(\mathrm{CH}), 131.4(\mathrm{CH}), 131.0(\mathrm{CH}), 130.9(\mathrm{CH}), 130.8(\mathrm{CH}), 130.5(\mathrm{CH}), 123.9(\mathrm{C}), 123.1(\mathrm{C})$, $122.7(\mathrm{C}), 121.8(\mathrm{C}), 121.0(\mathrm{C}), 120.92$ (C), 120.2 (C), $112.6(\mathrm{CH}), 91.2(\mathrm{C}), 90.8(\mathrm{C}), 90.6(\mathrm{C}), 89.5$ (C), $89.2(\mathrm{C}), 88.9(\mathrm{C}), 83.1(\mathrm{C}), 78.9(\mathrm{C}), 64.8(\mathrm{C}), 62.7(\mathrm{C}), 55.0\left(\mathrm{CH}_{3}\right)$.

Dendrimer G2b. A Schlenk tube equipped with a stirring bar was charged with freshly prepared 9 (340 mg, $0.17 \mathrm{mmol}), 5$ (33 mg, $0.037 \mathrm{mmol})$, CuI (1 mg, $0.0074 \mathrm{mmol}), \mathrm{Ph}_{3} \mathrm{P}$ (2 mg, $\left.0.0074 \mathrm{mmol}\right)$ and $\left(\mathrm{Ph}_{3} \mathrm{P}\right)_{4} \mathrm{Pd}(12 \mathrm{mg}, 0.015 \mathrm{mmol})$. After vacuum-drying, activated $4 \AA$ molecular sieves and piperidine $(20 \mathrm{~mL})$ were added under argon. The Schlenk tube was sealed with a teflon screw cap and the reaction mixture was stirred at $100{ }^{\circ} \mathrm{C}$ during $12 \mathrm{~h}$. Then it was allowed to reach rt. A white precipitate was formed, which was filtered and washed with refluxing $\mathrm{CH}_{2} \mathrm{Cl}_{2} / \mathrm{hexanes}$ and EtOAc/hexanes to give G2b as a poorly soluble white solid $(200 \mathrm{mg}, 0.024 \mathrm{mmol}, 64 \%, \mathrm{Rf}=0.54(20 \%$ toluene/ $\left.\mathrm{CH}_{2} \mathrm{Cl}_{2}\right) .{ }^{1} \mathrm{H} \mathrm{NMR}\left(\mathrm{CDCl}_{3}, 250 \mathrm{MHz}\right): 7.54-7.36(128 \mathrm{H}, \mathrm{m}, \mathrm{Ar}), 7.19(64 \mathrm{H}, \mathrm{m}, \mathrm{Ar}), 7.08(72 \mathrm{H}$, d, J=7.4 Hz, Ar $\left.\mathrm{OMe}_{\mathrm{O}}\right), 6.79\left(72 \mathrm{H}, \mathrm{d}, J=7.4 \mathrm{~Hz} \mathrm{Ar}_{\mathrm{OMe}}\right), 3.79(108 \mathrm{H}, \mathrm{s}, \mathrm{OMe}) .{ }^{13} \mathrm{C} \mathrm{NMR}\left(\mathrm{CDCl}_{3}, 63 \mathrm{MHz}\right)$ : $157.4(\mathrm{C}), 148.1(\mathrm{C}), 145.9(\mathrm{C}), 138.8(\mathrm{C}), 131.8(\mathrm{CH}), 131.4(\mathrm{CH}), 131.0(\mathrm{CH}), 130.9(\mathrm{CH}), 130.7$ (CH), $130.5(\mathrm{CH}), 123.1(\mathrm{C}), 122.9(\mathrm{C}), 122.7(\mathrm{C}), 121.0(\mathrm{C}), 120.2(\mathrm{C}), 112.6(\mathrm{CH}), 91.2(\mathrm{C}), 90.9(\mathrm{C})$, 90.7 (C), 90.6 (C), 89.5 (C), 88.9 (C), 64.6 (C), 62.7 (C), $55.0\left(\mathrm{CH}_{3}\right)$. MS (MALDI-TOF, m/z): 17074 $\left(\mathrm{M}_{2} \mathrm{Ag}^{+}\right), 8591\left(\mathrm{MAg}^{+}\right), 8375\left(\mathrm{M}^{+}-\mathrm{C}_{7} \mathrm{H}_{7} \mathrm{O}\right), 7872\left(\mathrm{M}^{+}-\mathrm{C}_{44} \mathrm{H}_{33} \mathrm{O}_{3}\right)$.

Protected dendron 12 from 11. A Schlenk tube equipped with a stirring bar was charged with $\mathbf{1 1}^{11 \mathrm{~b}}$ (190 mg, $0.24 \mathrm{mmol}), 6^{14}$ (70 mg, $\left.0.36 \mathrm{mmol}\right), \mathrm{CuI}$ (4 mg, $\left.0.047 \mathrm{mmol}\right), \mathrm{Ph}_{3} \mathrm{P}(12 \mathrm{mg}, 0.024 \mathrm{mmol})$ and $\left(\mathrm{Ph}_{3} \mathrm{P}\right)_{4} \mathrm{Pd}(27 \mathrm{mg}, 0.024 \mathrm{mmol})$. After vacuum-drying, activated $4 \AA$ molecular sieves and piperidine 
$(15 \mathrm{~mL})$ were added under argon. The Schlenk tube was sealed with a teflon screw cap and the reaction mixture was stirred at $100{ }^{\circ} \mathrm{C}$ during $12 \mathrm{~h}$ and then it was allowed to reach rt. The mixture was concentrated and the residue was dissolved in $\mathrm{CH}_{2} \mathrm{Cl}_{2}(30 \mathrm{~mL})$ and washed with a saturated solution of $\mathrm{NH}_{4} \mathrm{Cl}(3 \times 20 \mathrm{~mL})$. The organic layer was dried, filtered and concentrated. The residue was dissolved in $\mathrm{CH}_{2} \mathrm{Cl}_{2}$, and the product was precipitated with hexanes. The precipitate was filtered and reprecipitated three times to give 12 as a yellow solid [120 mg, $0.14 \mathrm{mmol}, 60 \%$ ]. ${ }^{1} \mathrm{H} \mathrm{NMR}\left(\mathrm{CDCl}_{3}, 250 \mathrm{MHz}\right): 7.41$ (4H, s, Ar), $7.36(2 \mathrm{H}, \mathrm{d}, J=7.5 \mathrm{~Hz}, \mathrm{Ar}), 7.28(6 \mathrm{H}, \mathrm{d}, J=7.5 \mathrm{~Hz}, \mathrm{Ar}), 7.22(2 \mathrm{H}, \mathrm{d}, J=7.5 \mathrm{~Hz}, \mathrm{Ar}), 7.16(6 \mathrm{H}$, d, J=7.5 Hz, Ar), $3.73\left(12 \mathrm{H}, \mathrm{s}, \mathrm{CH}_{2}-\mathrm{N}\right), 1.66\left(18 \mathrm{H}, \mathrm{s}, \mathrm{CH}_{2}\right), 0.23(9 \mathrm{H}, \mathrm{s}, \mathrm{TMS}) .{ }^{13} \mathrm{C} \mathrm{NMR}\left(\mathrm{CDCl}_{3}, 63\right.$ MHz): $148.5(\mathrm{C}), 147.8(\mathrm{C}), 143.8(\mathrm{C}), 131.7(\mathrm{CH}), 131.5(\mathrm{CH}), 131.2(\mathrm{CH}), 131.1(\mathrm{CH}), 130.5(\mathrm{CH})$, $123.3(\mathrm{C}), 122.5(\mathrm{C}), 120.1(\mathrm{C}), 119.3(\mathrm{CH}), 104.6(\mathrm{C}), 95.9(\mathrm{C}), 91.3(\mathrm{C}), 88.7(\mathrm{C}), 63.9(\mathrm{C}), 47.9$ (broad, $\left.\mathrm{CH}_{2}-\mathrm{N}\right), 25.1\left(\mathrm{CH}_{2}\right), 24.3\left(\mathrm{CH}_{2}\right), 0.00$ (TMS). MS (EI, m/z, \%): $849.2\left(\mathrm{M}^{+}, 7\right), 737.9\left(\mathrm{M}^{+}-\right.$ $\left.\mathrm{C}_{5} \mathrm{H}_{10} \mathrm{~N}_{3}, 15\right), 514.9\left[\mathrm{M}^{+}-3 \times\left(\mathrm{C}_{5} \mathrm{H}_{10} \mathrm{~N}_{3}\right), 100\right], 438.9$ (68). HRMS (EI) calcd. for $\mathrm{C}_{53} \mathrm{H}_{59} \mathrm{~N}_{9} \mathrm{Si}$ $\left(\mathrm{M}^{+}\right)$849.4663, found 849.4655.

Protected dendron 12 from 10. A Schlenk tube equipped with a stirring bar was charged with $\mathbf{1 0}^{11 \mathrm{~b}}$ (88 mg, $0.13 \mathrm{mmol}), 2^{12}$ (66 mg, $\left.0.26 \mathrm{mmol}\right), \mathrm{CuI}(3 \mathrm{mg}, 0.014 \mathrm{mmol}), \mathrm{Ph}_{3} \mathrm{P}(7 \mathrm{mg}, 0.026 \mathrm{mmol})$ and $\left(\mathrm{Ph}_{3} \mathrm{P}\right)_{4} \mathrm{Pd}(15 \mathrm{mg}, 0.014 \mathrm{mmol})$. After vacuum-drying, activated $4 \AA$ molecular sieves and piperidine (15 mL) were added under argon. The Schlenk tube was sealed with a teflon screw cap and the reaction mixture was stirred at $100{ }^{\circ} \mathrm{C}$ during $12 \mathrm{~h}$ and then it was allowed to reach $\mathrm{rt}$. The mixture was concentrated and the residue was dissolved in $\mathrm{CH}_{2} \mathrm{Cl}_{2}(20 \mathrm{~mL})$ and washed with a saturated solution of $\mathrm{NH}_{4} \mathrm{Cl}(3 \times 10 \mathrm{~mL})$. The organic layer was dried, filtered and concentrated. The residue was dissolved in $\mathrm{CH}_{2} \mathrm{Cl}_{2}$, and the product was precipitated with hexanes. The precipitate was filtered and reprecipitated 
three times to give 12 as a yellow solid [90 $\mathrm{mg}, 0.11 \mathrm{mmol}, 81 \%$ ]. Its spectroscopical data were identical to those of the product obtained from the previous experiment.

Dendron 13. To a solution of $12(268 \mathrm{mg}, 0.32 \mathrm{mmol})$ in $\mathrm{CH}_{2} \mathrm{Cl}_{2}(15 \mathrm{~mL})$ and $\mathrm{MeOH}(2 \mathrm{~mL})$ was added $\mathrm{KOH}(177 \mathrm{mg}, 3.15 \mathrm{mmol})$ under argon and the mixture was stirred at $\mathrm{rt}$ during $1 \mathrm{~h}$. The mixture was concentrated and the residue was dissolved in $\mathrm{CH}_{2} \mathrm{Cl}_{2}(30 \mathrm{~mL})$ and the solution was washed with brine $(3 \times 20 \mathrm{~mL})$, dried and concentrated. The resulting residue was dissolved in $\mathrm{CH}_{2} \mathrm{Cl}_{2}$ followed by precipitation with hexanes to give $\mathbf{1 3}$ as a brown solid [240 mg, $0.31 \mathrm{mmol}, 98 \%, \mathrm{Rf}=0.46(20 \%$ EtOAc/hexanes)]. This dendron was immediately used in next reaction. ${ }^{1} \mathrm{H} \mathrm{NMR}\left(\mathrm{CDCl}_{3}, 250 \mathrm{MHz}\right)$ : 7.50-7.11 (20H, m, Ar), $3.67\left(12 \mathrm{H}, \mathrm{s}, \mathrm{CH}_{2}-\mathrm{N}\right), 3.16(1 \mathrm{H}, \mathrm{s}, \mathrm{C} \equiv \mathrm{C}-\mathrm{H}), 1.66\left(18 \mathrm{H}, \mathrm{s}, \mathrm{CH}_{2}\right) .{ }^{13} \mathrm{C} \mathrm{NMR}$ $\left(\mathrm{CDCl}_{3}, 63 \mathrm{MHz}\right)$ : $148.6(\mathrm{C}), 147.9(\mathrm{C}), 143.8(\mathrm{C}), 131.9(\mathrm{CH}), 131.5(\mathrm{CH}), 131.3(\mathrm{CH}), 131.1(\mathrm{CH})$, $130.6(\mathrm{CH}), 123.8(\mathrm{C}), 121.6(\mathrm{C}), 120.0(\mathrm{C}), 119.4(\mathrm{CH}), 91.4(\mathrm{C}), 88.6(\mathrm{C}), 83.2(\mathrm{C}), 78.8(\mathrm{CH}), 63.9$ (C), 48.1 (broad, $\left.\mathrm{CH}_{2}-\mathrm{N}\right), 25.1\left(\mathrm{CH}_{2}\right), 24.3\left(\mathrm{CH}_{2}\right)$.

Dendrimer G1b-N3. A Schlenk tube equipped with a stirring bar was charged with freshly prepared 13 (240 mg, $0.31 \mathrm{mmol}), 5^{11,13}$ (56 mg, $\left.0.068 \mathrm{mmol}\right), \mathrm{CuI}(5 \mathrm{mg}, 0.027 \mathrm{mmol}), \mathrm{Ph}_{3} \mathrm{P}(11 \mathrm{mg}, 0.041$ mmol) and $\left(\mathrm{Ph}_{3} \mathrm{P}\right)_{4} \mathrm{Pd}(32 \mathrm{mg}, 0.027 \mathrm{mmol})$ and then the mixture was dried under vacuum. Activated $4 \AA$ molecular sieves and piperidine $(20 \mathrm{~mL})$ were added under argon and the Schlenk tube was sealed with a teflon screw cap. The reaction mixture was stirred at $100{ }^{\circ} \mathrm{C}$ during $12 \mathrm{~h}$ and then it was allowed to reach rt. The mixture was concentrated and the residue was dissolved in $\mathrm{CH}_{2} \mathrm{Cl}_{2}(30 \mathrm{~mL})$ and the solution was washed with a solution of $\mathrm{NH}_{4} \mathrm{Cl}(3 \times 10 \mathrm{~mL})$. The organic phase was dried and concentrated and the residue was purified by column GPC (Bio-Beads S-X1, THF) and precipitation from THF. The precipitate was filtered and washed with refluxing hexanes and then with refluxing $\mathrm{MeOH}$ to give G1bN3 as a brown solid (150 mg, $0.044 \mathrm{mmol}, 64 \%) .{ }^{1} \mathrm{H} \mathrm{NMR}\left(\mathrm{CDCl}_{3}, 400 \mathrm{MHz}\right): 7.60-7.00$ (96H, m, Ar), 
$3.75\left(48 \mathrm{H}, \mathrm{s}, \mathrm{CH}_{2}-\mathrm{N}\right), 1.69\left(72 \mathrm{H}, \mathrm{s}, \mathrm{CH}_{2}\right) .{ }^{13} \mathrm{C} \mathrm{NMR}\left(\mathrm{CDCl}_{3}, 63 \mathrm{MHz}\right): 148.5$ (C), 147.9 (C), 144.1

(CH), $143.8(\mathrm{CH}), 131.5(\mathrm{CH}), 131.0(\mathrm{CH}), 130.5(\mathrm{CH}), 119.3(\mathrm{CH}), 63.9(\mathrm{C}), 48.0\left(\right.$ broad, $\left.\mathrm{CH}_{2}-\mathrm{N}\right)$, $25.1\left(\mathrm{CH}_{2}\right), 24.3\left(\mathrm{CH}_{2}\right)$.

Dendrimer G1b-I. A Schlenk tube equipped with a stirring bar was charged with G1b-N3 (70 mg, $0.020 \mathrm{mmol})$ and it was dried under vacuum. Then, MeI $(10 \mathrm{~mL})$ was slowly added under argon, the Schlenk tube was sealed with a teflon screw cap and the reaction mixture was stirred at $120{ }^{\circ} \mathrm{C}$ during 48 h. After reaching rt, the mixture was diluted with $\mathrm{CH}_{2} \mathrm{Cl}_{2}(50 \mathrm{~mL})$ and the resulting solution was washed with saturated solutions of $\mathrm{Na}_{2} \mathrm{~S}_{2} \mathrm{O}_{3}(3 \times 10 \mathrm{~mL})$ and $\mathrm{NH}_{4} \mathrm{Cl}(3 \times 10 \mathrm{~mL})$. The organic phase was dried and concentrated and the poorly soluble residue was washed with refluxing hexanes and then with $\mathrm{MeOH}$ to give G1b-I as a yellow solid (70 mg, $0.019 \mathrm{mmol}, 95 \%) .{ }^{1} \mathrm{H} \mathrm{NMR}\left(\mathrm{CDCl}_{3}, 250 \mathrm{MHz}\right): 8.0-$ $7.33\left(64 \mathrm{H}, \mathrm{m}, \mathrm{Ar} / \mathrm{Ar}_{\mathrm{I}}\right), 7.13(8 \mathrm{H}, \mathrm{d}, J=7.3 \mathrm{~Hz}, \mathrm{Ar}), 6.90\left(24 \mathrm{H}, \mathrm{d}, J=7.9 \mathrm{~Hz}, \mathrm{Ar}_{\mathrm{I}}\right) .{ }^{13} \mathrm{C} \mathrm{NMR}\left(\mathrm{CDCl}_{3}, 63\right.$ MHz): $145.0(\mathrm{C}), 136.9(\mathrm{CH}), 132.5(\mathrm{CH}), 131.8(\mathrm{CH}), 131.4(\mathrm{CH}), 131.0(\mathrm{CH}), 130.9(\mathrm{CH}), 130.5$ (CH), $129.6(\mathrm{CH}), 92.3(\mathrm{C}), 64.4(\mathrm{C}), 63.7$ (C). MS (MALDI-TOF, m/z): $2980\left(\mathrm{M}^{+}-\mathrm{C}_{19} \mathrm{H}_{12} \mathrm{I}_{3}\right), 2361\left[\mathrm{M}^{+}-\right.$ $\left.2 \mathrm{x}\left(\mathrm{C}_{19} \mathrm{H}_{12} \mathrm{I}_{3}\right)\right], 1741\left[\mathrm{M}^{+}-3 \mathrm{x}\left(\mathrm{C}_{19} \mathrm{H}_{12} \mathrm{I}_{3}\right)\right]$.

Dendrimer G1c. Piperidine $(0.2 \mathrm{~mL}, 2.3 \mathrm{mmol})$ was added to a solution $\mathbf{1}^{11}(100 \mathrm{mg}, 0.23 \mathrm{mmol})$, $\mathrm{CuI}(10 \mathrm{mg}, 0.46 \mathrm{mmol})$ and $\left(\mathrm{Ph}_{3} \mathrm{P}\right)_{4} \mathrm{Pd}(5 \mathrm{mg}, 0.0046 \mathrm{mmol})$ in $\mathrm{THF}(10 \mathrm{~mL})$ and the mixture was stirred at $\mathrm{rt}$ for $2 \mathrm{~h}$ in an open round bottom flask. The mixture was concentrated, the residue was dissolved in EtOAc $(20 \mathrm{~mL})$ and was washed with a solution of $\mathrm{NH}_{4} \mathrm{Cl}(3 \times 15 \mathrm{~mL})$. The organic phase was dried and concentrated and the residue was purified by flash chromatography $\left(\mathrm{SiO}_{2}, 15 \%\right.$ EtOAc/hexanes) to give G1c as a white solid $[98 \mathrm{mg}, 0.075 \mathrm{mmol}, 98 \%, \quad \mathrm{Rf}=0.4 \quad(30 \%$ EtOAc/hexanes)]. ${ }^{1} \mathrm{H} \mathrm{NMR}\left(\mathrm{CDCl}_{3}, 250 \mathrm{MHz}\right): 7.40$ (4H, d, J=8.4 Hz, Ar), 7.18 (4H, d, J=8.4 Hz, Ar), $7.07\left(12 \mathrm{H}, \mathrm{d}, J=8.9 \mathrm{~Hz}, \mathrm{Ar}_{\mathrm{OMe}}\right), 6.79\left(12 \mathrm{H}, \mathrm{d}, J=8.9 \mathrm{~Hz} \mathrm{Ar}_{\mathrm{OMe}}\right), 3.79(18 \mathrm{H}, \mathrm{s}, \mathrm{OMe}) .{ }^{13} \mathrm{C} \mathrm{NMR}\left(\mathrm{CDCl}_{3}\right.$, 
$63 \mathrm{MHz})$ : $157.7(\mathrm{C}), 149.2(\mathrm{C}), 138.9(\mathrm{C}), 132.1(\mathrm{CH}), 131.7(\mathrm{CH}), 131.1(\mathrm{CH}), 119.2(\mathrm{C}), 112.9(\mathrm{CH})$,

81.7 (C), 74.0 (C), 63.0 (C), $55.3\left(\mathrm{CH}_{3}\right) . \mathrm{MS}(\mathrm{EI}, \mathrm{m} / \mathrm{z}, \%): 866\left(\mathrm{M}^{+}, 40\right), 759\left(\mathrm{M}^{+}-\mathrm{C}_{7} \mathrm{H}_{7} \mathrm{O}, 39\right), 333$ $\left(\mathrm{C}_{22} \mathrm{H}_{21} \mathrm{O}_{3}{ }^{+}, 100\right)$. MS (EI, m/z, \%): $893.2\left(\mathrm{M}^{+}, 100\right), 690.4\left(\mathrm{M}^{+}-\mathrm{C}_{6} \mathrm{H}_{5} \mathrm{I}, 67\right)$. HRMS (EI) calcd. For $\mathrm{C}_{60} \mathrm{H}_{50} \mathrm{O}_{6}^{+}\left(\mathrm{M}^{+}\right)$866.3607, found 866.3607.

Compound 15. A Schlenk tube equipped with a stirring bar was charged with freshly prepared $\mathbf{1}^{11}$ (250 mg, $0.58 \mathrm{mmol}), 1^{6}$ (118 mg, $\left.0.17 \mathrm{mmol}\right)$, CuI (6 mg, $\left.0.033 \mathrm{mmol}\right), \mathrm{Ph}_{3} \mathrm{P}(9 \mathrm{mg}, 0.033 \mathrm{mmol}) \mathrm{y}$ $\left(\mathrm{Ph}_{3} \mathrm{P}\right)_{4} \mathrm{Pd}(57 \mathrm{mg}, 0.050 \mathrm{mmol})$ and then the mixture was dried under vacuum. Activated $4 \AA$ molecular sieves and piperidine $(20 \mathrm{~mL})$ were added under argon and the Schlenk tube was sealed with a teflon screw cap. The reaction mixture was stirred at $100{ }^{\circ} \mathrm{C}$ during $12 \mathrm{~h}$ and then it was allowed to reach $\mathrm{rt}$. The mixture was concentrated and the residue was dissolved in $\mathrm{CH}_{2} \mathrm{Cl}_{2}(20 \mathrm{~mL})$ and the solution was washed with a solution of $\mathrm{NH}_{4} \mathrm{Cl}(3 \times 15 \mathrm{~mL})$. The organic phase was dried and concentrated and the residue was purified by flash chromatography $\left(\mathrm{SiO}_{2}, 30 \% \mathrm{EtOAc} /\right.$ hexanes) to give $\mathbf{1 5}$ as a yellow solid [196 mg, $0.12 \mathrm{mmol}, 73 \%, \mathrm{Rf}=0.42$ (40\% EtOAc/hexanes)]. ${ }^{1} \mathrm{H} \mathrm{NMR}\left(\mathrm{CDCl}_{3}, 250 \mathrm{MHz}\right): 7.38(12 \mathrm{H}$, m, Ar), $7.19(12 \mathrm{H}, \mathrm{d}, J=8.3 \mathrm{~Hz}, \mathrm{Ar}), 7.10\left(22 \mathrm{H}, \mathrm{m}, \mathrm{Ar}_{\mathrm{OMe} / \mathrm{OH}}\right), 6.80\left(20 \mathrm{H}, \mathrm{d}, J=8.7 \mathrm{~Hz}, \operatorname{Ar}_{\mathrm{OMe}}\right), 6.74(2 \mathrm{H}$, d, J=9.1 Hz, $\left.\mathrm{Ar}_{\mathrm{OH}}\right) 3.80(27 \mathrm{H}, \mathrm{s}, \mathrm{OMe}) .{ }^{13} \mathrm{C} \mathrm{NMR}\left(\mathrm{CDCl}_{3}, 63 \mathrm{MHz}\right): 157.4(\mathrm{C}), 147.9$ (C), $146.4(\mathrm{C})$, $138.9(\mathrm{C}), 132.0(\mathrm{CH}), 131.9(\mathrm{CH}), 131.1(\mathrm{CH}), 130.8(\mathrm{CH}), 130.5(\mathrm{CH}), 121.0(\mathrm{C}), 120.4(\mathrm{C}), 112.6$ (CH), $89.4(\mathrm{C}), 88.9(\mathrm{C}), 62.6(\mathrm{C}), 55.1\left(\mathrm{CH}_{3}\right)$. MS (MALDI-TOF, m/z): $1632.6\left(\mathrm{M}^{+}\right)$.

Compound 16. A solution of $15(120 \mathrm{mg}, 0.073 \mathrm{mmol})$ and $\mathrm{Et}_{3} \mathrm{~N}(0.036 \mathrm{~mL}, 0.256 \mathrm{mmol})$ in dry $\mathrm{CH}_{2} \mathrm{Cl}_{2}(20 \mathrm{~mL})$ was cooled to $-78{ }^{\circ} \mathrm{C}$ during $10 \mathrm{~min}$. Then, $\mathrm{Tf}_{2} \mathrm{O}(0.024 \mathrm{~mL}, 0.147 \mathrm{mmol})$ was added and the mixture was allowed to reach $\mathrm{rt}$ for $12 \mathrm{~h}$. The mixture was washed with a saturated solution of $\mathrm{NH}_{4} \mathrm{Cl}(40 \mathrm{~mL})$ and the aqueous phase was extracted with EtOAc $(3 \times 30 \mathrm{~mL})$. The combined organic phase was dried and concentrated and the residue was purified by flash chromatography $\left(\mathrm{SiO}_{2}, 20 \%\right.$ 
EtOAc/hexanes) to give $\mathbf{1 6}$ as a white solid [122 $\mathrm{mg}, 0.069 \mathrm{mmol}, 95 \%, \mathrm{Rf}=0.65 \quad(40 \%$

EtOAc/hexanes)]. ${ }^{1} \mathrm{H}$ NMR $\left(\mathrm{CDCl}_{3}, 250 \mathrm{MHz}\right): 7.46-7.38(12 \mathrm{H}, \mathrm{m}, \mathrm{Ar}), 7.30\left(2 \mathrm{H}, \mathrm{d}, J=8.9 \mathrm{~Hz}, \mathrm{Ar}_{\mathrm{OTf}}\right)$, 7.25-7.10 (14H, m, Ar), $7.07\left(18 \mathrm{H}, \mathrm{d}, \mathrm{d}, J=8.9 \mathrm{~Hz}, \mathrm{Ar}_{\text {Оме }}\right), 6.77\left(18 \mathrm{H}, \mathrm{d}, J=8.9 \mathrm{~Hz}, \mathrm{Ar}_{\text {Оме }}\right), 3.75(27 \mathrm{H}, \mathrm{s}$, OMe). ${ }^{13} \mathrm{C} \mathrm{NMR}\left(\mathrm{CDCl}_{3}, 63 \mathrm{MHz}\right): 157.4(\mathrm{C}), 148.0(\mathrm{C}), 145.3(\mathrm{C}), 138.9(\mathrm{C}), 132.5(\mathrm{CH}), 131.8(\mathrm{CH})$, $131.1(\mathrm{CH}), 130.8(\mathrm{CH}), 130.5(\mathrm{CH}), 121.5(\mathrm{C}), 120.5(\mathrm{CH}), 120.2(\mathrm{C}), 112.6(\mathrm{CH}), 89.8(\mathrm{C}), 88.5(\mathrm{C})$, $62.8(\mathrm{C}), 55.0\left(\mathrm{CH}_{3}\right)$. MS (MALDI-TOF, m/z): $1764.5\left(\mathrm{M}^{+}\right)$.

Protected dendron 17. A Schlenk tube equipped with a stirring bar was charged with 16 (116 g, $0.066 \mathrm{mmol}), \mathrm{CuI}(1 \mathrm{mg}, 0.0066 \mathrm{mmol}), \mathrm{Ph}_{3} \mathrm{P}(3 \mathrm{mg}, 0.0131 \mathrm{mmol})$ y $\left(\mathrm{Ph}_{3} \mathrm{P}\right)_{4} \mathrm{Pd}(8 \mathrm{mg}, 0.0066 \mathrm{mmol})$. After vacuum-drying, activated $4 \AA$ molecular sieves, trimethylsilylacetilene $(0.056 \mathrm{~mL}, 0.39 \mathrm{mmol})$ and piperidine $(15 \mathrm{~mL})$ were successively added under argon. The Schlenk tube was sealed with a teflon screw cap and the reaction mixture was stirred at $100{ }^{\circ} \mathrm{C}$ during $12 \mathrm{~h}$ and then it was allowed to reach $\mathrm{rt}$. The mixture was concentrated and the residue was dissolved in $\mathrm{CH}_{2} \mathrm{Cl}_{2}(20 \mathrm{~mL})$ and washed with a saturated solution of $\mathrm{NH}_{4} \mathrm{Cl}(3 \times 10 \mathrm{~mL})$. The organic layer was dried, filtered and concentrated and the residue was purified by flash chromatography $\left(\mathrm{SiO}_{2}, 20 \% \mathrm{CH}_{2} \mathrm{Cl}_{2} /\right.$ hexanes $)$ to give $17^{11 \mathrm{la}}$ as a white solid [107 mg, $0.062 \mathrm{mmol}, 95 \%, \mathrm{Rf}=0.42$ (30\% EtOAc/hexanes)]. Its spectroscopic data were identical to those of a reference sample.

Dendrimer G2c. Piperidine $(0.04 \mathrm{~mL}, 0.47 \mathrm{mmol})$ was added to a solution of 18 (153 $\mathrm{mg}, 0.093$ mmol prepared from 17 as previously described by our group $\left.{ }^{11 \mathrm{a}}\right), \mathrm{CuI}(3 \mathrm{mg}, 0.19 \mathrm{mmol})$ and $\left(\mathrm{Ph}_{3} \mathrm{P}\right)_{4} \mathrm{Pd}$ (22 $\mathrm{mg}, 0.019 \mathrm{mmol})$ in THF $(10 \mathrm{~mL})$ and the mixture was stirred at $\mathrm{rt}$ for $2 \mathrm{~h}$ in an open round bottom flask. The mixture was concentrated, the residue was dissolved in EtOAc $(20 \mathrm{~mL})$ and was washed with a solution of $\mathrm{NH}_{4} \mathrm{Cl}(3 \times 15 \mathrm{~mL})$. The organic phase was dried and concentrated and the residue was purified by flash chromatography $\left(\mathrm{SiO}_{2}, 25 \%\right.$ EtOAc/hexanes) to give $\mathbf{G 2 c}$ as a white solid [150 mg, 
$0.046 \mathrm{mmol}, 98 \%, \mathrm{Rf}=0.45\left(80 \% \mathrm{CH}_{2} \mathrm{Cl}_{2} /\right.$ hexanes $\left.)\right] .{ }^{1} \mathrm{H} \mathrm{NMR}\left(\mathrm{CDCl}_{3}, 250 \mathrm{MHz}\right): 7.42(28 \mathrm{H}, \mathrm{m}, \mathrm{Ar})$, $7.19(28 \mathrm{H}, \mathrm{d}, J=8.1 \mathrm{~Hz}, \mathrm{Ar}), 7.10\left(36 \mathrm{H}, \mathrm{d}, J=8.8 \mathrm{~Hz}, \operatorname{Ar}_{\text {OMe }}\right), 6.80\left(36 \mathrm{H}, \mathrm{d}, J=8.8 \mathrm{~Hz} \operatorname{Ar}_{\text {OMe }}\right), 3.80(54 \mathrm{H}$, s, OMe). ${ }^{13} \mathrm{C} \mathrm{NMR}\left(\mathrm{CDCl}_{3}, 63 \mathrm{MHz}\right): 157.6$ (C), 148.2 (C), 147.2 (C), 145.7 (C), 139.17 (C), 132.1 $(\mathrm{CH}), 131.2(\mathrm{CH}), 131.0 \mathrm{CH}), 130.9(\mathrm{CH}), 130.8(\mathrm{CH}), 121.6(\mathrm{C}), 120.6(\mathrm{C}), 119.9(\mathrm{C}), 112.9(\mathrm{CH})$, 89.9 (C), 88.9 (C), 81.5 (C), 74.4 (C), 65.0 (C), 62.7 (C), $55.3\left(\mathrm{CH}_{3}\right)$. MS (MALDI-TOF, m/z): 3389 $\left(\mathrm{MAg}^{+}\right), 3280\left(\mathrm{M}^{+}\right), 3174\left(\mathrm{M}^{+}-\mathrm{C}_{7} \mathrm{H}_{7} \mathrm{O}\right), 2772\left(\mathrm{M}^{+}-\mathrm{C}_{36} \mathrm{H}_{29} \mathrm{O}_{3}\right), 1540\left(\mathrm{M}^{+}-\mathrm{C}_{109} \mathrm{H}_{87} \mathrm{O}_{9}\right)$.

Dendrimer G1c-N3. Piperidine $(0.110 \mathrm{~mL}, 1.3 \mathrm{mmol})$ was added to a solution of $\mathbf{1 0}^{1 \mathrm{lb}}(88 \mathrm{mg}, 0.13$ $\mathrm{mmol}), \mathrm{CuI}(50 \mathrm{mg}, 0.26 \mathrm{mmol})$ and $\left(\mathrm{Ph}_{3} \mathrm{P}\right)_{4} \mathrm{Pd}(15 \mathrm{mg}, 0.013 \mathrm{mmol})$ in THF $(10 \mathrm{~mL})$ and the mixture was stirred at $\mathrm{rt}$ for $2 \mathrm{~h}$ in an open round bottom flask. The mixture was concentrated, the residue was dissolved in $\mathrm{CH}_{2} \mathrm{Cl}_{2}(10 \mathrm{~mL})$ and was washed with a solution of $\mathrm{NH}_{4} \mathrm{Cl}(3 \times 10 \mathrm{~mL})$. The organic phase was dried and diluted with hexanes until precipitation of a solid. The precipitate was filtered and dried to give G1c-N3 (85 mg, $0.063 \mathrm{mmol}, 97 \%) .{ }^{1} \mathrm{H} \mathrm{NMR}\left(\mathrm{CDCl}_{3}, 250 \mathrm{MHz}\right): 7.35$ (4H, d, J=8.0 Hz, Ar), 7.27 $(12 \mathrm{H}, \mathrm{d}, J=8.3 \mathrm{~Hz}, \mathrm{Ar}), 7.20(4 \mathrm{H}, \mathrm{d}, J=8.0 \mathrm{~Hz}, \mathrm{Ar}), 7.14(12 \mathrm{H}, \mathrm{d}, J=8.3 \mathrm{~Hz}, \mathrm{Ar}), 3.72\left(24 \mathrm{H}, \mathrm{s}, \mathrm{CH}_{2}-\mathrm{N}\right)$, $1.65\left(36 \mathrm{H}, \mathrm{s}, \mathrm{CH}_{2}\right) .{ }^{13} \mathrm{C} \mathrm{NMR}\left(\mathrm{CDCl}_{3}, 63 \mathrm{MHz}\right): 148.6(\mathrm{C}), 143.6(\mathrm{C}), 131.4(\mathrm{CH}), 131.0(\mathrm{CH}), 119.4$ $(\mathrm{CH}), 119.0(\mathrm{C}), 81.4(\mathrm{C}), 73.8(\mathrm{C}), 64.0(\mathrm{C}), 48.0\left(\right.$ broad, $\left.\mathrm{CH}_{2}-\mathrm{N}\right), 25.1\left(\mathrm{CH}_{2}\right), 24.2\left(\mathrm{CH}_{2}\right)$.

Dendrimer G1c-I. A Schlenk tube equipped with a stirring bar was charged with G1c-N3 (8 mg, $0.059 \mathrm{mmol})$ and it was dried under vacuum. Then, MeI $(5 \mathrm{~mL})$ was slowly added under argon, the Schlenk tube was sealed with a teflon screw cap and the reaction mixture was stirred at $120{ }^{\circ} \mathrm{C}$ during 48 h. After reaching rt, the mixture was diluted with $\mathrm{CH}_{2} \mathrm{Cl}_{2}(50 \mathrm{~mL})$ and the resulting solution was washed with saturated solutions of $\mathrm{Na}_{2} \mathrm{~S}_{2} \mathrm{O}_{3}(3 \times 10 \mathrm{~mL})$ and $\mathrm{NH}_{4} \mathrm{Cl}(3 \times 10 \mathrm{~mL})$. The organic phase was dried and concentrated and the poorly soluble residue was washed with refluxing hexanes and then with $\mathrm{MeOH}$ to give G1c-I as a yellow solid (70 mg, $0.059 \mathrm{mmol}, 82 \%) .{ }^{1} \mathrm{H} \mathrm{NMR}\left(\mathrm{CDCl}_{3}, 250 \mathrm{MHz}\right): 7.58$ 
$\left(12 \mathrm{H}, \mathrm{d}, J=8.5 \mathrm{~Hz}, \mathrm{Ar}_{\mathrm{I}}\right), 7.41(4 \mathrm{H}, \mathrm{d}, J=8.3 \mathrm{~Hz}, \mathrm{Ar}), 7.11(4 \mathrm{H}, \mathrm{d}, J=8.5 \mathrm{~Hz}, \mathrm{Ar}), 6.88(12 \mathrm{H}, \mathrm{d}, J=8.5 \mathrm{~Hz}$ $\left.\operatorname{Ar}_{\mathrm{I}}\right)$.

Dendrimer G3c. A Schlenk tube equipped with a stirring bar was charged with freshly prepared G1cI (15 mg, $0.010 \mathrm{mmol}), 18$ (152 $\mathrm{mg}, 0.093 \mathrm{mmol}$, prepared from 17 as previously described by our group $\left.^{11 \mathrm{a}}\right), \mathrm{CuI}(2 \mathrm{mg}, 0.0063 \mathrm{mmol}), \mathrm{Ph}_{3} \mathrm{P}(2 \mathrm{mg}, 0.0063 \mathrm{mmol})$ y $\left(\mathrm{Ph}_{3} \mathrm{P}\right)_{4} \mathrm{Pd}(8 \mathrm{mg}, 0.0073 \mathrm{mmol})$. After vacuum-drying, activated $4 \AA$ molecular sieves and piperidine $(15 \mathrm{~mL})$ were added under argon. The Schlenk tube was sealed with a teflon screw cap and the reaction mixture was stirred at $100{ }^{\circ} \mathrm{C}$ during $12 \mathrm{~h}$ and then it was allowed to reach $\mathrm{rt}$. The resulting mixture was concentrated and the residue was dissolved in $\mathrm{CH}_{2} \mathrm{Cl}_{2}(20 \mathrm{~mL})$ and washed with a saturated solution of $\mathrm{NH}_{4} \mathrm{Cl}(3 \times 15 \mathrm{~mL})$. The organic layer was dried and concentrated to give a residue, which was purified by column GPC (Bio-Beads SX1, THF) and precipitation from THF solution. The precipitate was filtered and washed with refluxing hexanes and methanol to give dendrimer G3c as a white solid [ $88 \mathrm{mg}, 0.0084 \mathrm{mmol}, 80 \%, \mathrm{Rf}=0.55$ (10\% hexanes/ $\left.\mathrm{CH}_{2} \mathrm{Cl}_{2}\right)$ ]. ${ }^{1} \mathrm{H}$ NMR $\left(\mathrm{CDCl}_{3}, 250 \mathrm{MHz}\right): 7.41$ (100H, m, Ar), 7.19 (100H, m, CH-Ar), $7.09(108 \mathrm{H}, \mathrm{d}, J=8.7 \mathrm{~Hz}, \mathrm{Ar}), 6.79\left(108 \mathrm{H}, \mathrm{d}, J=8.7 \mathrm{~Hz}, \mathrm{Ar}_{\mathrm{OMe}}\right), 3.78(162 \mathrm{H}, \mathrm{s}, \mathrm{OMe}) .{ }^{13} \mathrm{C} \mathrm{NMR}\left(\mathrm{CDCl}_{3}\right.$, $63 \mathrm{MHz})$ : 157.4 (C), 147.9 (C), 145.9 (C), 145.7 (C), 145.7 (C), $138.9(\mathrm{C}), 131.8(\mathrm{CH}), 130.9(\mathrm{CH})$, $130.8(\mathrm{CH}), 130.7(\mathrm{CH}), 130.5(\mathrm{CH}), 121.2(\mathrm{C}), 121.0(\mathrm{C}), 120.3(\mathrm{C}), 112.6(\mathrm{CH}), 89.6(\mathrm{C}), 89.3(\mathrm{C})$, $89.1(\mathrm{C}), 88.7(\mathrm{C}), 64.7(\mathrm{C}), 62.7(\mathrm{C}), 55.0\left(\mathrm{CH}_{3}\right)$. MS (MALDI-TOF, m/z): $10636.8\left(\mathrm{MAg}^{+}\right)$.

Acknowledgment. We acknowledge financial support from Spanish MINECO (grant MAT201342425-R) and MICINN (grant PGC2018-101047-B-I00), co-funding from FEDER, and a predoctoral fellowship from the Chile Government to J.I.U. (CONICYT Becas Chile).

Supporting Information Available: Copies of NMR spectra (PDF). 


\section{References}

1 (a) S. Sengupta, S. K. Sadhukhan, Tetrahedron Lett. 1998, 39, 1237-1238. (b) N. Armaroli, V.

Balzani, J. P. Collin, P. Gavina, J. P. Sauvage, B. Ventura, J. Am. Chem. Soc. 1999, 121, 4397-4408. (c)

M. R. Robinson, S. Wang, G. C. Bazan, Y. Cao, Adv. Mater. 2000, 12, 1701-1704. (d) M. Quintiliani, A. Kahnt, P. Vásquez, D. M. Guldi, T. Torres, J. Mater. Chem. 2008, 18, 1542-1546. (e) T. Muller, S. Bräse, RSC Adv. 2014, 4, 6886.

2 (a) B. F. Hoskins, R. Robson, J. Am. Chem. Soc. 1990, 112, 1546-1554. (b) D. Venkataraman, S.

Lee, J. S. Moore, P. Zhang, K. A. Hirsch, G. B. Gardner, A. C. Corey, C. L. Prentice, Chem. Mater.

1996, 8, 2030-2040. (c) A. Tanatini, T. S. Hughes, J. S. Moore, Angew. Chem. Int. Ed. Engl. 2002, 41, 325-328. (d) J. H. Ryu, H. J. Kim, Z. Huang, E. Lee, M. Lee, Angew. Chem. Int. Ed. 2006, 45, 53045307. (e) M. Quintiliani, A. Kahnt, P. Vásquez, D. M. Guldi, T. Torres, J. Mater. Chem. 2008, 18, 15421546. (f) J. Tian, Y. Ding, T. Zhou, K. Zhang, X. Zhao, H. Wang, D. Zhang, Y. Liu, Z. Li, Chem. Eur. J. 2014, 20, 575-584.

3 (a) S. Wang, W. J. Jr. Oldham, R. A. Jr. Hudack, G. C. Bazan, J. Am. Chem. Soc. 2000, 122, 5695-5709. (b) X. M. Liu, C. He, J. Huang, J. W. Xu, Chem. Mater. 2005, 17, 434-441. (c) C. Lee. J. Lee, Chem. Eur. J. 2012, 18, 6457-6461.

4 (a) S. Wang, W. J. Jr. Oldham, R. A. Jr. Hudack, G. C. Bazan, J. Am. Chem. Soc. 2000, 122, 5695-5709. (b) L. H. Chan, R. H. Lee, C. F. Hsieh, H. C. Yeh, C. T. J. Chen, J. Am. Chem. Soc. 2002, 124, 6469-6479. (c) X. M. Liu, C. He, J. Huang, J. W. Xu, Chem. Mater. 2005, 17, 434-441. (d) K. 
Tsuchiya, M. Ueda. Polym. J. 2006, 38, 956-960. (e) M. Quintiliani, A. Kahnt, P. Vázquez, D. M. Guldi, T. Torres, J. Mater. Chem. 2008, 18, 1542-1546. (f) C. Lee. J. Lee, Chem. Eur. J. 2012, 18, 6457-6461.

5 (a) A. Pegenau, T. Hegmann, C. Tschierske, S. Diele, Chem. Eur. J. 1999, 5, 1643-1660. (b) T. Hatano, T. Kato, Tetrahedron 2008, 64, 8368-8380.

6 M. E. Gallina, B. Baytekin, C. Schalley, P. Ceroni, Chem. Eur. J. 2012, 18, 1528-1535.

7 (a) U. M. Wiesler, A. J. Berresheim, F. Morgenroth, G. Lieser, K. Mullen, Macromolecules 2001, 34, 187-199. (b) M. Schlupp, T. Weil, A. J. Berresheim, U. M. Wiesler, J. Bargon, K. Müllen, Angew. Chem. Int. Ed. 2001, 40, 4011-4015. (c) B. A. G. Hammer, R. Moritz, R. Stangenberg, M. Baumgarten, K. Müllen, Chem. Soc. Rev. 2015, 44, 4072-4090. (d) M. Brutschy, R. Stangenberg, C. Beer, D. Lubczyk, M. Baumgarten, K. Müllen, S. R. Waldwogel, ChemPlusChem 2015, 80, 54-56.

8 Y. Jin, A. Jin, R. McCaffrey, H. Long, W. Zhang, J. Org. Chem. 2012, 77, 7392-7400.

9 (a) J. Kim, B. Chen, T. M. Reineke, H. Li, M. Eddaoudi, D. B. Moler, M. O’Keeffe, O. M. Yaghi. J. Am. Chem. Soc. 2001, 123, 8239-8247. (b) A. P. Côté, A. I. Benin, N. W. Ockwig, M. O’Keeffe, A. J. Matzger, O. M. Yaghi, Science, 2005, 310, 1166-1170. (c) H. M. El-Kaderi, J. R. Hunt, J. L. MendozaCortés, A. P. Côté, R. E. Taylor, M. O’Keeffe, O. M. Yaghi, Science, 2007, 316, 268-272. (d) F. J. UribeRomo, J. R. Hunt, H. Furukawa, C. Klöck, M. O’Keeffe, O. M. Yaghi, J. Am. Chem. Soc. 2009, 131, 4570-4571. (e) D. Beaudoin, T. Maris, J. D. Wuest, Nat. Chem. 2013, 5, 830-834. (f) Q. Fang, J. Wang, S. Gu, R. B. Kaspar, Z. Zhuang, J. Zheng, H. Guo, S. Qiu, Y. Yan, J. Am. Chem. Soc. 2015, 137, 83528355. 
10 (a) E. Stöckel, X. Wu, A. Trewin, C. D. Wood, R. Clowes, N. L. Campbell, J. T. A. Jones, Y. Z.

Khimyak, D. J. Adams, A. I. Cooper, Chem. Commun. 2009, 212-214. (b) O. K. Farha, A. M. Spokoyny,

B. G. Hauser, Y.-S. Bae, S. E. Brown, R. Q. Snurr, C. A. Mirkin, J. T. Hupp, Chem. Mater. 2009, 21, 3033-3035. (c) T. Ben, H. Ben, S. Ma, D. Cao, J. Lan, X. Jing, W. Wang, J. Xu, F. Deng, J. M. Simmons, S. Qiu, G. Zhu, Angew. Chem. Int. Ed. 2009, 48, 9457-9460. (d) J. R. Holst, E. Stöckel, D. J. Adams, A. I. Cooper, Macromolecules 2010, 43, 8531-8538. (e) Y. Zhu, H. Yang, Y. Ji, W. Zhang, Chem. Mater. 2013, 25, 3718-3723.

11 (a) J. I. Urzúa, M. A. Regueira, M. Lazzari, M. Torneiro, Polym. Chem. 2016, 7, 5641-5645. (b)

J. I. Urzúa, M. Torneiro, J. Org. Chem. 2017, 82, 13231-13238.

12 U. Ziener, A. Godt, J. Org. Chem. 1997, 62, 6137-6143.

13 (a) Q. Li, A. V. Rukavishnikov, P. A. Petukhov, T. O. Zaikova, , J. F. W. Keana Org. Lett. 2002, 4, 3631-3634. (b) D. Su, F. M. Menger, Tetrahedron Lett. 1997, 38, 1485-1488.

14 J. G. Rodríguez, J. L. Tejedor, T. La Parra, C. Díaz, Tetrahedron 2006, 62, 3355-3361.

15 C. Glaser, Ber. Dtsch. Chem. Ges. 1869, 2, 422-424.

16 K. Balaraman, V. Kesavan, Synthesis 2010, 20, 3461-3466.

17 Q. Zheng, R. Hua, Y. Wan, Appl. Organomet. Chem. 2010, 24, 314-316.

18 For general procedures see reference 11a. 
